Aus der Abteilung Anaesthesiologie

(Prof. Dr. med. M. Quintel)

im Zentrum Anaesthesiologie, Rettungs- und Intensivmedizin

der Medizinischen Fakultät der Universität Göttingen

\title{
Untersuchungen zur notärztlichen Versorgung von Kindernotfällen
}

\author{
Charakteristik, Wahrnehmung und Einschätzung
}

\author{
Inaugural-Dissertation \\ zur Erlangung des Doktorgrades \\ der Medizinischen Fakultät \\ der Georg-August-Universität zu Göttingen
}

vorgelegt von

Uta Gentkow

aus Berlin 
Dekan: Prof. Dr. med. C. Frömmel

I. Berichterstatter: Prof. Dr. med. M. Quintel

II. Berichterstatter/in:

III. Berichterstatter/in:

Tag der mündlichen Prüfung: 


\section{Inhalt}

\section{Abkürzungsverzeichnis}

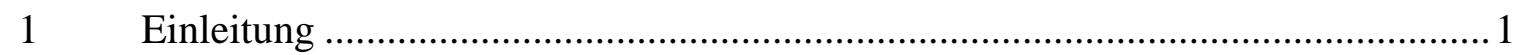

1.1 Einführung in das Thema ,präklinische Notfallmedizin` ....................................... 1

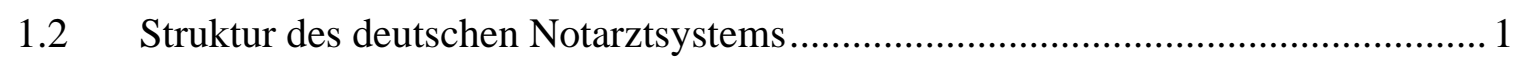

1.3 Potentielle Defizite im gegenwärtigen Notarztsystem ......................................... 2

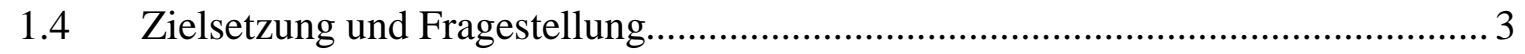

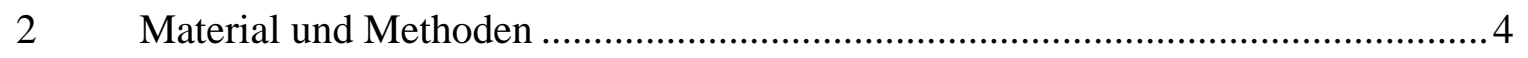

$2.1 \quad$ Struktur des Notarztdienstes in Göttingen.......................................................... 4

2.2 Einschätzung des Schweregrades der Notfälle...................................................... 5

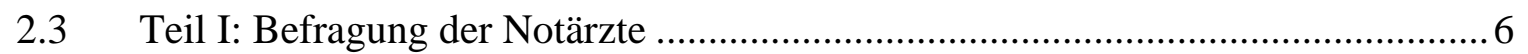

2.4 Teil II: Gewinnung und Auswertung der RED-Daten.......................................... 7

2.5 Methodisches Vorgehen und statistische Auswertung ......................................... 9

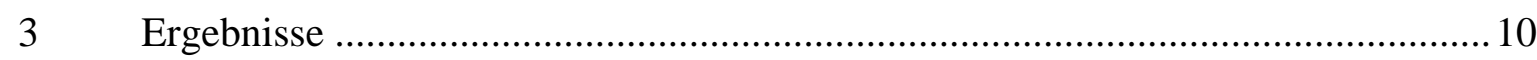

3.1 Teil I: Analyse der Befragung der Notärzte ................................................... 10

3.1.1 Einschätzung der Häufigkeit pädiatrischer Notfälle............................................ 10

3.1.2 Einschätzung des Schweregrades präklinischer pädiatrischer Notfälle .................. 12

3.1.3 Einschätzung der Häufigkeit von Verdachtsdiagnosen .......................................... 12

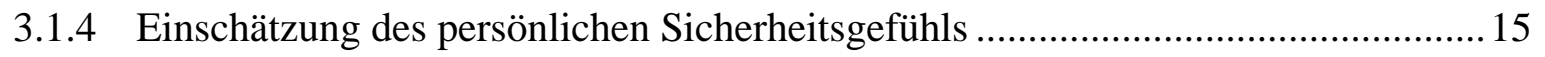

3.1.5 Persönliche Erfahrung mit präklinischen Kindernotfällen ................................... 18

3.2 Teil II: Ergebnisse der Analyse der Rettungsdiensteinsatzdokumentation (RED) .22

3.2.1 Differenzierung nach dem Rettungsmittel......................................................2 22

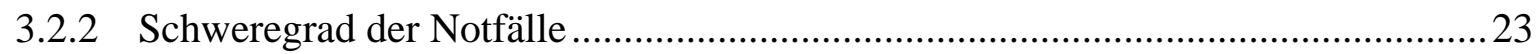

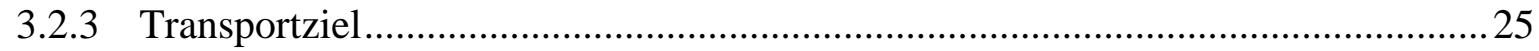

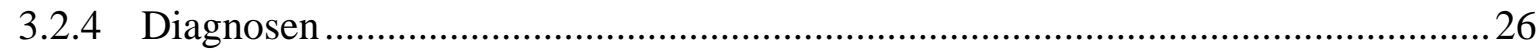

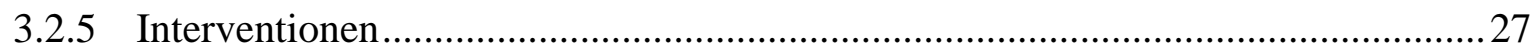

3.2.6 Diagnosen und Interventionen in Abhängigkeit vom Rettungsmittel .....................28

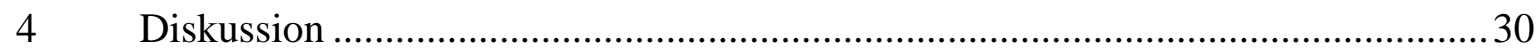

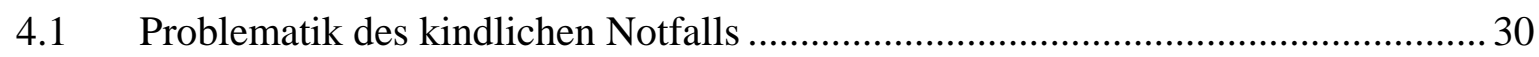

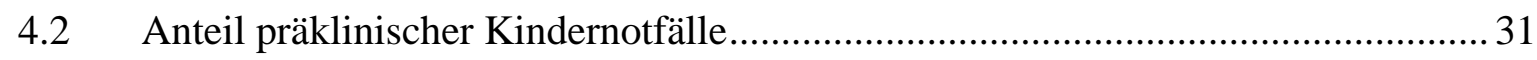

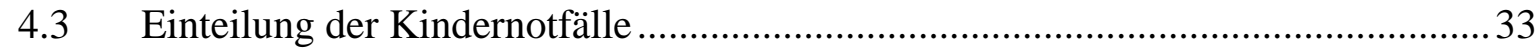


4.3.1 Abgrenzung pädiatrischer Patienten von erwachsenen Patienten ...........................33

4.3.2 Abgrenzung der Entwicklungsperioden des Kindes...............................................33

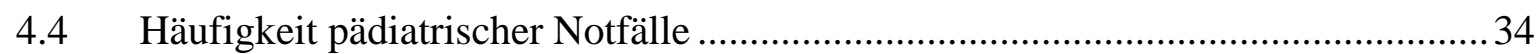

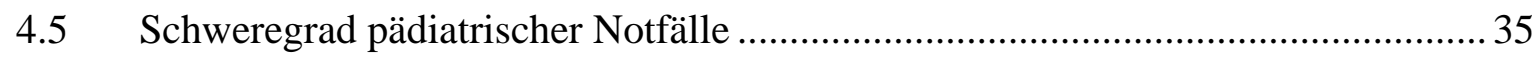

4.6 Sicherheit im Umgang mit den Altersgruppen ................................................ 37

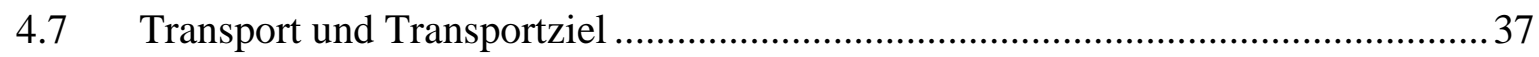

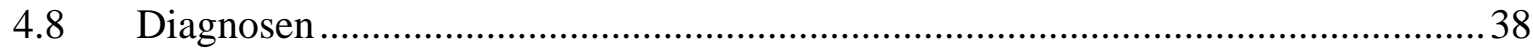

4.8.1 Häufigkeit pädiatrischer Notfalldiagnosen............................................................. 38

4.8.2 Häufigkeiten pädiatrischer Notfalldiagnosen in den Altersgruppen .......................39

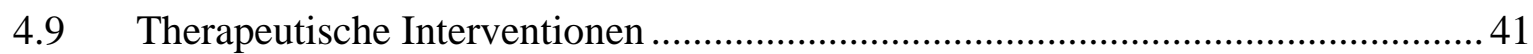

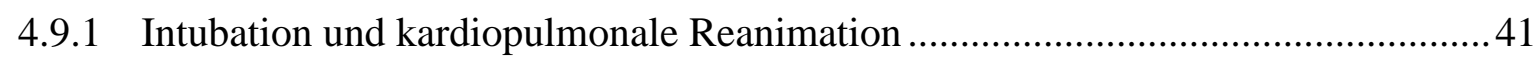

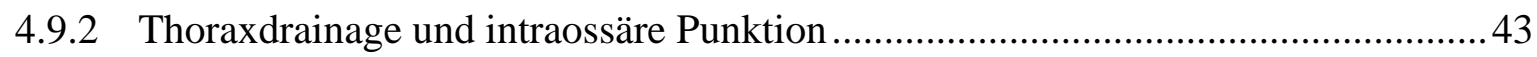

4.10 Einschätzung von Diagnosen und Interventionen .................................................. 44

4.10.1 Häufigkeiten von Diagnosen und Interventionen.................................................... 44

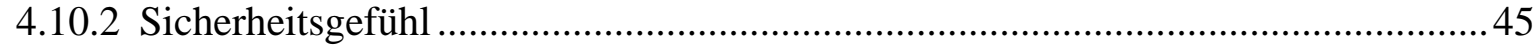

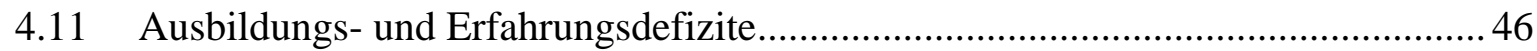

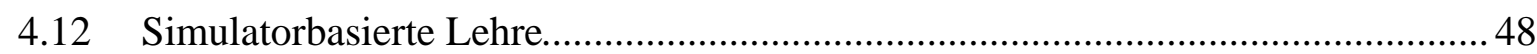

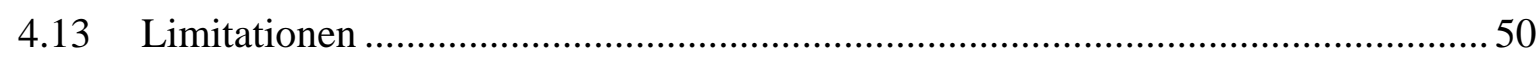

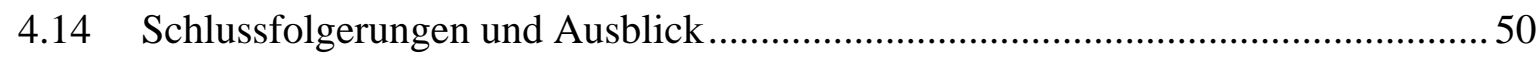

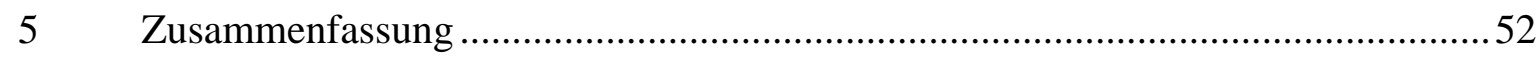

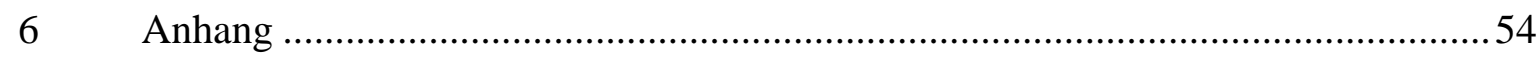

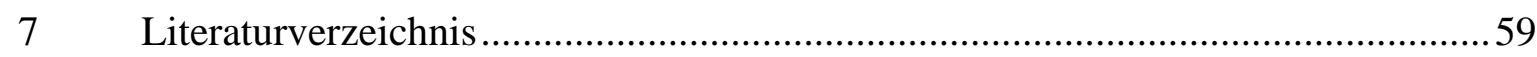




\section{Abkürzungsverzeichnis}

\begin{tabular}{|c|c|}
\hline a & Annus (Jahr) \\
\hline Amb & Ambulanz \\
\hline CPR & Kardiopulmonale Reanimation \\
\hline $\begin{array}{l}\text { CRM } \\
\text { DIVI } \\
\text { EPLS }\end{array}$ & $\begin{array}{l}\text { Crisis Resource Management (Zwischenfallsmanagement) } \\
\text { Deutsche Interdisziplinäre Vereinigung für Intensiv- und Notfallmedizin } \\
\text { European Paediatric Life Support }\end{array}$ \\
\hline ERC & European Resuscitation Council \\
\hline $\begin{array}{l}\text { FBAO } \\
\text { Heli }\end{array}$ & $\begin{array}{l}\text { Foreign Body Airway Obstruction (Fremdkörperaspiration) } \\
\text { Helikopter }\end{array}$ \\
\hline $\begin{array}{l}\text { ILCOR } \\
\text { i.o. }\end{array}$ & $\begin{array}{l}\text { International Liaison Committee on Resuscitation } \\
\text { intraossär }\end{array}$ \\
\hline i.v. & intravenös \\
\hline LJ & Lebensjahre \\
\hline LMA & Laryngeal Mask Airway (Larynxmaske) \\
\hline $\mathrm{m}$ & männlich \\
\hline $\mathrm{n}$ & Anzahl \\
\hline $\mathrm{n} / \mathrm{a}$ & Nicht verfügbar/vorhanden \\
\hline $\begin{array}{l}\text { NACA } \\
\text { NLS }\end{array}$ & $\begin{array}{l}\text { National Advisory Committee on Aeronautics } \\
\text { Newborn Life Support }\end{array}$ \\
\hline NAW & Notarztwagen \\
\hline NEF & Notarzteinsatzfahrzeug \\
\hline PALS & Paediatric Advanced Life Support \\
\hline PBLS & Paediatric Basic Life Support \\
\hline RED & Rettungsdiensteinsatzdokumentationssystem \\
\hline RTH & Rettungstransporthubschrauber \\
\hline SHT & Schädel-Hirn-Trauma \\
\hline $\begin{array}{l}\text { SIDS } \\
\mathrm{w}\end{array}$ & $\begin{array}{l}\text { Sudden Infant Death Syndrome (Plötzlicher Kindstod) } \\
\text { weiblich }\end{array}$ \\
\hline WB & Weiterbildung \\
\hline ZARI & $\begin{array}{l}\text { Zentrum Anaesthesiologie, Rettungs- und Intensivmedizin der Universitätsmedi- } \\
\text { zin Göttingen }\end{array}$ \\
\hline ZNS & Zentrales Nervensystem \\
\hline
\end{tabular}




\section{Einleitung}

\subsubsection{Einführung in das Thema präklinische Notfallmedizin}

Ein medizinischer Notfall ist ein Akutereignis, welches durch eine vitale Gefährdung für den Patienten ${ }^{*}$ gekennzeichnet ist und somit eine unverzügliche Hilfeleistung notwendig macht. Die präklinische Notfallmedizin hat in Deutschland eine lange Tradition. Während sich der Fokus in den Anfangszeiten primär auf die Behandlung verletzter Patienten richtete, um deren Morbidität und Mortalität zu reduzieren, übernahm der Rettungsdienst im Verlauf zunehmend Verantwortung auch für die Versorgung akut erkrankter Patienten, z. B. bei Herz-KreislaufStillstand (Burghofer und Lackner 2006).

Heute erreicht ein genormt ausgestattetes Rettungsmittel, welches bei Bedarf auch einen Notarzt beinhaltet, nahezu jeden Einsatzort in der Bundesrepublik Deutschland innerhalb einer gesetzlichen Hilfsfrist von 15 Minuten (Roessler und Zuzan 2006). Nach Stopfkuchen zählt das deutsche Rettungsdienstsystem weltweit $\mathrm{zu}$ den leistungsfähigsten seiner Art (Stopfkuchen et al. 1999). Dies gilt sowohl für die technische Ausstattung und Logistik des Rettungsdienstes, als auch für die Präsenz und Qualität des ärztlichen und nicht-ärztlichen Personals.

\subsection{Struktur des deutschen Notarztsystems}

Das Spektrum an Erkrankungen und Verletzungen, das ein Notarzt versorgen muss, setzt sich aus allen existierenden medizinischen Fachbereichen zusammen. Dabei machen internistische und neurologische Notfälle das Gros der Patienten aus, während der Anteil der traumatologischen Notfälle im Laufe der Jahre stetig abgenommen hat (Gries et al. 2005, Knapp et al. 2008). Da es in Deutschland, so wie in den meisten Ländern Europas, keinen Facharzt für Notfallmedizin gibt, übernehmen Ärzte unterschiedlicher klinischer Disziplinen die Versorgung von Notfallpatienten (Stenke 2004, Roessler und Zuzan 2006). Dabei muss sich der Notarzt auf sein medizinisches Wissen, seine praktischen Fertigkeiten, seine Erfahrung sowie auf die reibungslose Unterstützung durch sein Rettungsteam verlassen können. Eine erfolgreiche kardiopulmonale Reanimation zum Beispiel erfordert korrekte, zeitgerechte und sichere Ausführung bestimmter technischer Fertigkeiten sowie effektives Teammanagement und gute Kommunikation (Wheeler et al. 2003).

Im Folgenden wird versucht, möglichst geschlechtsneutral zu formulieren. Der Singular sowie der Plural der betreffenden Personen schließen beide natürlichen Geschlechter ein. Das Gleiche gilt im Folgenden für Arzt/Ärztin, Notarzt/Notärztin usw. 
Präklinische Kindernotfälle stellen einen Notarzt häufig vor besonders große fachliche und emotionale Herausforderungen (Zink et al. 2004). Im Rahmen der inzwischen bundesweit eingeführten Zusatzbezeichnung bzw. Fachkunde Notfallmedizin sind aber vor allem explizit praktische Kenntnisse und Fertigkeiten der Kindernotfallmedizin nur in sehr geringem Maße berücksichtigt. Es ist daher nicht verwunderlich, dass viele Notärzte über Unsicherheit oder gar Angst gerade bei Kindernotfällen berichten (Bartels 2001, Knapp 1995, Zink et al. 2004).

Notärzte rekrutieren sich in Deutschland aus unterschiedlichen Fachrichtungen, vor allem aus der Anästhesiologie, der Inneren Medizin, der Chirurgie und der Allgemeinmedizin. Die entsprechenden Anteile variieren nach lokalen und regionalen Gegebenheiten. Auch die gesetzlichen Voraussetzungen zur aktiven Teilnahme am Notarztdienst sind in den einzelnen Bundesländern sehr unterschiedlich geregelt. In Niedersachsen sind folgende Anforderungen zu erfüllen: 30 Monate klinische Praxis, davon mindestens sechs Monate in der Intensivmedizin, Anästhesie oder Notaufnahme eines Krankenhauses, ein 80-Stunden-Kurs nach dem Mustercurriculum Notfallmedizin der Bundesärztekammer, 20 lebensrettende Einsätze unter Supervision eines weiterbildungsermächtigten Notarztes sowie die bestandene Prüfung zur Zusatzbezeichnung Notfallmedizin (Roessler und Zuzan 2006, ÄK Niedersachsen 2004). Eine interne Regelung des Zentrums Anaesthesiologie, Rettungs- und Intensivmedizin der Universitätsmedizin Göttingen sieht darüber hinaus vor, dass zumindest alle in der Luftrettung tätigen Notärzte zusätzlich eine viermonatige Rotation in der Kinderanästhesie abgeschlossen haben müssen.

Auf pädiatrische Notfälle spezialisierte Notarztteams sind im deutschen Rettungsdienst - wie auch in anderen Ländern Europas - nur in wenigen deutschen Großstädten etabliert (Stenke 2004). Der Großteil kritischer präklinischer Kindernotfälle wird demnach durch nicht-spezialisierte Notärzte versorgt (Eich et al. 2006, Roessler und Zuzan 2006). Diese haben oft nur geringe Erfahrungen im Umgang mit kranken oder verletzten Kindern. Notärzte ohne fundierte kindernotfallmedizinische Kenntnisse und Fertigkeiten können dabei schnell überfordert sein (Gries et al. 2005, Stenke 2004).

\subsection{Potentielle Defizite im gegenwärtigen Notarztsystem}

Ein Rettungsteam sieht sich insbesondere bei präklinischen Kindernotfällen mit einem hohen Erfolgsdruck konfrontiert (Rossi 1999). Die damit häufig verbundene Diskrepanz zwischen hohen medizinischen und organisatorischen Anforderungen einerseits und geringer klinischer Routine andererseits wird zusätzlich verschärft durch eine fortschreitende Zentralisierung der 
erweiterten pädiatrischen Versorgung, durch die Reduktion von Arbeitszeiten sowie durch die weitgehende Subspezialisierung (Eich et al. 2006). Weiterhin üben viele Helfer aus Angst vor Schaden große Zurückhaltung hinsichtlich der Durchführung dringender Behandlungsmaßnahmen bei kritisch kranken Kindern aus (Eich et al. 2007a). Es ist zudem bekannt, dass Helfer bei Kindernotfällen immer wieder auf lebensrettende Maßnahmen verzichten, da sie Angst haben, etwas verkehrt zu machen (Francis et al. 2008, Biarent et al. 2005). Es stellt sich daher die Frage, ob die Versorgungsqualität bei präklinischen Kindernotfällen schlechter ist als bei Erwachsenennotfällen (Knapp 1995, Stenke 2004). Inmitten dieses Spannungsfeldes muss sich der Notarzt trotzdem der Herausforderung stellen, präklinische Kindernotfälle adäquat zu versorgen - eine Aufgabe, auf die er oft unzureichend vorbereitet ist (Bartels 2001, Knapp 1995, Zink et al. 2004).

Die aktuellen Leitlinien zu den lebensrettenden Maßnahmen bei Kindern des European Resuscitation Council (ERC) wurden 2005 publiziert (Biarent et al. 2005). Basierend auf dem wissenschaftlichen Konsensus des International Liaison Committee on Resuscitation, (ILCOR) wurde neben der bestmöglichen Evidenz vor allem Wert auf die breite Umsetzbarkeit der Leitlinien gelegt (ILCOR 2005a, ILCOR 2005b). Die deutsche Notfallmedizin schien sich mit der Akzeptanz und Umsetzung internationaler Leitlinien, welche auch als Basis einer kontinuierlichen Qualitätssicherung in der präklinischen Versorgung pädiatrischer Patienten dienen können, lange schwer zu tun (Gries und Wilhelm 2004). Kritik an der Qualität veröffentlichter Empfehlungen und unklare juristische Verbindlichkeiten erschweren noch immer deren Akzeptanz und praktische Umsetzung (Ballnus et al. 2002). Dabei stellen gerade Notfälle im Kindesalter auch heute noch, im Zeitalter der „Hightech-Medizin“, eine besondere Problematik dar (Brambrink 1999).

\subsection{Zielsetzung und Fragestellung}

Ziel der dieser Dissertationsschrift zugrunde liegenden Untersuchungen war es, die besonderen Umstände und Charakteristika präklinischer Kindernotfälle und ihrer Teilaspekte realistisch zu analysieren, die daraus resultierenden Implikationen für die notärztliche Versorgung akut erkrankter oder verletzter Kinder zu benennen und daraus schlussfolgernd mögliche Lösungsstrategien für eine verbesserte Aus-, Weiter- und Fortbildung aufzuzeigen. Ein besonderes Augenmerk richtete sich dabei auf die Frage, wie sich anästhesiologisch weitergebildete Notärzte den Anforderungen bei der notfallmedizinischen Versorgung von Kindern stellen. 


\section{Material und Methoden}

Nach formaler Genehmigung der Untersuchung durch die Ethik-Kommission der Medizinischen Fakultät der Georg-August-Universität Göttingen wurde im Zeitraum vom 1. Juli bis zum 31. Dezember 2006 ein Fragebogen zur Wahrnehmung und Einschätzung präklinischer Kindernotfälle an alle 50 zu diesem Zeitpunkt boden- und luftgebundenen notärztlich tätigen Ärzte des Zentrums Anaesthesiologie, Rettungs- und Intensivmedizin (ZARI) der Universitätsmedizin Göttingen verteilt (Teil I). Die Beantwortung der Fragen und der Rücklauf der Fragebögen erfolgten anonym.

Weiterhin wurden alle im Rettungseinsatzdokumentationssystem ${ }^{\circledR}\left(\right.$ RED $^{\circledR}$, TIM GmbH, Göttingen, Deutschland) des Rettungsdienstbereichs Göttingen erfassten, präklinischen Kindernotfälle (Alter unter 15 Jahre), die im Zeitraum vom 1. Januar 1998 bis 31. Dezember 2006 von einem Notarzt des Zentrums Anaesthesiologie, Rettungs- und Intensivmedizin versorgt wurden, retrospektiv ausgewertet (Teil II).

\subsection{Struktur des Notarztdienstes in Göttingen}

Im Rettungsdienstbereich Göttingen ist die präklinische Versorgung von Kindernotfällen Aufgabe des allgemeinen Rettungsdienstes. Es gibt keinen spezialisierten Kindernotarztdienst („Kindernotarzt“). Die Bereitstellung der Notärzte erfolgt ausschließlich durch das Zentrum Anaesthesiologie, Rettungs- und Intensivmedizin (ZARI) der Universitätsmedizin Göttingen. Deren Einzugsbereich erstreckt sich - inklusive der Luftrettung - über etwa 6.000 Quadratkilometer mit ca. 600.000 Einwohnern.

Rund um die Uhr werden zwei arztbesetzte Rettungsmittel bereitgestellt: Tagsüber ein Notarzteinsatzfahrzeug (NEF) und ein Rettungstransporthubschrauber (RTH, Christoph 44); nach Sonnenuntergang statt des RTH ein zweites NEF. Bei Bedarf ist ein weiterer Notarzt von den anästhesiologischen Intensivstationen der Universitätsmedizin Göttingen abrufbar. Diese Notarztstruktur ist im Bedarfsplan der Träger des Rettungsdienstes für die Stadt und den Landkreis Göttingen festgelegt.

Abhängig von Art und Ausmaß der Erkrankung oder Verletzung, der Tageszeit sowie der Verfügbarkeit der einzelnen Rettungsmittel wird von der integrierten Feuerwehr- und Rettungsleitstelle der Berufsfeuerwehr Göttingen entweder ein bodengebundener (NEF) oder ein luftgebundener (RTH) Notarzt eingesetzt. Der RTH kann zudem von benachbarten Leitstellen 
angefordert werden. Pro Jahr werden von den in Göttingen stationierten Notärzten über 4.000 Einsätze absolviert, davon ca. 1.400 luftgebunden.

Um im Rettungsdienstbereich Göttingen tätig zu sein, muss jeder Notarzt mindestens zwei Jahre klinische Anästhesie und ein Jahr intensivmedizinisches Training absolviert haben. Weiterhin muss er das 80-stündige, zertifizierte Curriculum „Notfallmedizin“ (BÄK 2004) und ein Minimum von 20 supervidierten präklinischen Notarzteinsätzen absolviert haben. Schließlich muss er die Prüfung bei der zuständigen Ärztekammer zur Zusatzbezeichnung (Niedersachsen) bzw. Fachkunde (andere Bundesländer) „Notfallmedizin“ erfolgreich bestanden haben. Im luftgebundenen Rettungsdienst tätige Notärzte (RTH) müssen zudem eine fünfmonatige Vollzeittätigkeit im bodengebundenen Rettungsdienst (NEF) mit mindestens 300 versorgten Notfällen sowie eine viermonatige Rotation in der Kinderanästhesie absolviert haben.

\subsection{Einschätzung des Schweregrades der Notfälle}

Zur groben Einschätzung des Schweregrades von Verletzungen und Erkrankungen wird in der präklinischen Notfallmedizin der sogenannte NACA-Score (National Advisory Committee for Aeronautics) angewendet. Ursprünglich stammt der NACA-Score aus dem amerikanischen Militärwesen, wo er als Bewertungsmaßstab für die Dringlichkeit einer Luftrettung mittels Helikopter diente (Schlechtriemen et al. 2005b). Dabei wurden der NACA-S-Index (Severity of Injury or Illness) zur Wiedergabe der Befindlichkeit des Patienten und der NACA-L-Index (Location of Event) zur Definition der Beschaffenheit des Terrains sowie der Möglichkeit der Erreichbarkeit des Patienten unterschieden.

Nach mehreren Modifikationen wird der heute in der Notfallmedizin angewandte NACA-Score in sieben Kategorien untergeteilt, bei der eine allgemeine medizinische Definition sowie eine chirurgische Definition unterschieden werden (Tabelle 1). 


\begin{tabular}{|c|c|c|}
\hline NACA-Score & Chirurgisch & Medizinisch \\
\hline I & $\begin{array}{l}\text { Sehr geringfügige, leichte Verlet- } \\
\text { zung }\end{array}$ & $\begin{array}{l}\text { Nicht akut lebensbedrohliche Er- } \\
\text { krankung }\end{array}$ \\
\hline II & Mäßig schwere Verletzung & $\begin{array}{l}\text { Keine notärztlichen Maßnahmen, } \\
\text { jedoch klinische Diagnostik not- } \\
\text { wendig }\end{array}$ \\
\hline III & $\begin{array}{l}\text { Schwere, nicht gefährliche Verlet- } \\
\text { zung eines Körperteils }\end{array}$ & $\begin{array}{l}\text { Schwere, jedoch nicht lebensgefähr- } \\
\text { liche Erkrankung, notärztliche Maß- } \\
\text { nahmen wesentlich }\end{array}$ \\
\hline IV & $\begin{array}{l}\text { Schwere Verletzung an verschiede- } \\
\text { nen Körperteilen oder multiple Ver- } \\
\text { letzungen eines Körperteils, jedoch } \\
\text { keine vitale Gefährdung }\end{array}$ & $\begin{array}{l}\text { Entwicklung einer Vitalgefährdung } \\
\text { nicht auszuschließen }\end{array}$ \\
\hline V & $\begin{array}{l}\text { Schwere und lebensgefährliche Ver- } \\
\text { letzung eines Körperteils }\end{array}$ & Akute Vitalgefährdung \\
\hline VI & $\begin{array}{l}\text { Schwere und gefährliche Verletzun- } \\
\text { gen mehrerer Körperteile (Poly- } \\
\text { trauma) }\end{array}$ & $\begin{array}{l}\text { Akuter Atem- u./o. Kreislaufstill- } \\
\text { stand }\end{array}$ \\
\hline VII & Tod & Tod \\
\hline
\end{tabular}

Tabelle 1 Einteilung des NACA-Score (Klier 2003, S. 38)

\subsection{Teil I: Befragung der Notärzte}

Zur Gewinnung valider Daten wurde ein differenzierter Fragebogen entworfen, der Wahrnehmung und Einschätzung präklinischer pädiatrischer Notfälle durch aktive Notärzte erfassen sollte. Dieser wurde an alle 50 zu diesem Zeitpunkt notärztlich tätigen Ärzte des Zentrums Anaesthesiologie, Rettungs- und Intensivmedizin (ZARI) der Universitätsmedizin Göttingen ausgegeben.

Inhalt und Struktur des verwendeten Fragebogens basierten auf der Auswertung von Evaluationsbögen zahlreicher, im eigenen Zentrum angebotenen notfallmedizinischen Fortbildungsveranstaltungen (u. a. Kurs Notfallmedizin, European Paediatric Life Support(EPLS-) Kurs, Kindersimulationskurse und Notarzt-Refresher-Kurse) sowie auf den Datensätzen des Göttinger Rettungseinsatzdokumentationssystem ${ }^{\circledR}\left(\right.$ RED $^{\circledR}$, TIM GmbH, Göttingen, Deutschland). Zur weiteren Orientierung dienten thematisch verwandte Untersuchungen der notfallmedizinischen Literatur (Albrech et al. 2000, Ballnus et al. 2002, Bartels 2001, Gries et al. 2005, Schlechtriemen et al. 2005a). 


\author{
Persönliche Daten \\ Alter (25-30, 31-40, über 40 Lebensjahre) \\ Geschlecht \\ Ausbildungsstand (in Weiterbildung, Facharzt) \\ Erfahrung als Notarzt (<2 Lebensjahre, 2-5 Lebensjahre, $>5$ Lebensjahre) \\ Notärztliche Erfahrung (bodengebunden, luftgebunden)
}

\title{
Einschätzung des Anteils der Kindernotfälle [\%] im Rettungsdienstbereich Göttingern so- wie deren jeweilige NACA-Scores \\ Gesamt \\ Differenziert nach Altersgruppen: Säuglinge (0-1 Lebensjahr), Kleinkinder (1-5 Lebensjahre) und Schulkinder (6-14 Lebensjahre)
}

\section{Einschätzung der Inzidenz von Diagnosen und Interventionen}

(auf einer Skala von 1 (sehr selten) bis 10 (sehr häufig))

Differenziert nach Altersgruppen: Säuglinge (0-1 Lebensjahr), Kleinkinder (1-5 Lebensjahre) und Schulkinder (6-14 Lebensjahre)

\section{Persönliches Sicherheitsgefühl bei präklinischen Kindernotfällen}

(auf einer Skala von 1 (sehr unsicher) bis 10 ( sehr sicher))

Differenziert nach Altersgruppen: Neugeborene, Säuglinge (0-1 Lebensjahr), Kleinkinder (1-

5 Lebensjahre) und Schulkinder (6-14 Lebensjahre)

\section{Freitextantworten}

Persönliche Expertise im Umgang mit pädiatrischen Notfällen

Absolvierte pädiatrische Trainingskurse

Empfundene, persönliche Lehrdefizite

Potentielle Möglichkeiten zur Verbesserung der Aus-, Weiter- und Fortbildung sowie Definition von entsprechenden Lernzielen

Tabelle 2 Struktur und Inhalt des Fragebogens

\subsection{Teil II: Gewinnung und Auswertung der RED-Daten}

Alle patientenbezogenen Daten (Datum, Zeit und Ort des Einsatzes, boden-/luftgebundener Einsatz, Patientenalter, Geschlecht, Krankengeschichte, Verlauf der Vitalparameter, Verletzungen, Schweregrad der Verletzungen [NACA-Score], Einweisungsdiagnose) sowie Daten zu den am Patienten durchgeführten Interventionen werden routinemäßig sofort nach Beendigung der Versorgung des Patienten in einer elektronischen Notfalldatenbank, dem Rettungseinsatzdokumentationssystem $\left(\operatorname{RED}^{\circledR}\right)$, erfasst. Das RED ${ }^{\circledR}$ dient der medizinischen Dokumentation, der Abrechnung sowie der Forschung und Lehre und wird seit 1995 im Rettungsdienstbereich Göttingen verwendet. 
Mit Hilfe des elektronischen RED $^{\circledR}$-Protokolls führten wir eine retrospektive Analyse aller präklinischen pädiatrischen Notfälle durch, die im Zeitraum vom 1. Januar 1998 bis zum 31. Dezember 2006 von einem ärztlich besetzten Rettungsmittel des Rettungsdienstbereiches Göttingen versorgt wurden. Zunächst wurden die Datensätze aller pädiatrischen Notfälle identifiziert, indem die RED ${ }^{\circledR}$-Datenbank nach der Hauptkategorie „Pädiatrie“ sowie nach der Altersgruppe: „Alter < 15 Jahre“ durchsucht wurde. Zusätzlich wurde in der Datenbank eine Freitextsuche durchgeführt, um spezielle Diagnosen und Interventionen, z. B. Ertrinken, Epiglottitis, kardiopulmonale Reanimation, intraossäre Punktion und Einlage einer Thoraxdrainage vollständig und detailliert zu erfassen. Zur Plausibilitätsprüfung unklarer Datensätze wurden ebenfalls die Freitextangaben untersucht. Alle Daten wurden nach folgenden Kriterien differenziert (Tabelle 3).

\begin{tabular}{|c|c|}
\hline Differenzierung nach Altersgruppe & $\begin{array}{l}\text { 0-1 Lebensjahr (Säuglinge) } \\
\text { 1-5 Lebensjahre (Kleinkinder) } \\
\text { 6-14 Lebensjahre (Schulkinder) }\end{array}$ \\
\hline Differenzierung nach Diagnosen & $\begin{array}{l}\text { Respiratorische Störungen (gesamt) } \\
\text { Plötzlicher Kindstod (SIDS) } \\
\text { Ertrinken } \\
\text { Pseudokrupp } \\
\text { Epiglottitis } \\
\text { Asthma \& Bronchiolitis } \\
\text { Aspiration \& Fremdkörperaspiration } \\
\text { Andere } \\
\text { Trauma (gesamt) } \\
\text { Schädel-Hirn-Trauma (SHT) } \\
\text { Verbrennungen \& Verbrühungen } \\
\text { Andere } \\
\text { Krampfanfälle (gesamt) } \\
\text { Fieberkrämpfe } \\
\text { Andere } \\
\text { Andere Diagnosen }\end{array}$ \\
\hline Differenzierung nach Interventionen & $\begin{array}{l}\text { Endotracheale Intubation, LMA-Insertion } \\
\text { Intraossäre Punktion } \\
\text { Thoraxdrainage } \\
\text { Defibrillation } \\
\text { Kardiopulmonale Reanimation (CPR) }\end{array}$ \\
\hline Differenzierung nach NACA-Score & $\begin{array}{l}\text { NACA-Score I-III } \\
\text { NACA-Score IV-VII }\end{array}$ \\
\hline Differenzierung nach Rettungsmittel & $\begin{array}{l}\text { Bodengebunden (NEF) } \\
\text { Luftgebunden (RTH) }\end{array}$ \\
\hline Differenzierung nach Transportziel & $\begin{array}{l}\text { Universitätsklinikum Göttingen } \\
\text { Andere } \\
\text { Kein Transport }\end{array}$ \\
\hline
\end{tabular}




\subsection{Methodisches Vorgehen und statistische Auswertung}

Zur statistischen Auswertung der Fragebögen wurden die persönlichen Daten der teilnehmenden Notärzte (Alter, Geschlecht, Ausbildungsstand, notärztliche Erfahrung) in einer Excel ${ }^{\circledR}$ Tabelle (Microsoft, Seattle, USA) erfasst und verschlüsselt. Gleichermaßen wurde mit den jeweiligen persönlichen Einschätzungen von Häufigkeiten und Sicherheitsempfinden bezüglich Diagnosen und Interventionen in den drei Altersgruppen Säuglinge, Kleinkinder und Schulkinder verfahren. Diese Daten wurden dem jeweiligen Fragebogen zugeordnet.

Für die deskriptive Analyse erfolgte die Berechnung von Mittelwert (bzw. Median bei nicht-normalverteilten Daten), Standardabweichung, Minimum, Maximum, 25\%-, 50\%-, 75\%-Perzentile mittels SPSS Statistics 16.0.2 ${ }^{\circledR}$ (SPSS Inc., Chicago, USA). Bei fehlenden Daten erfolgte ein Ausschluss aus der statistischen Analyse. Die graphische und tabellarische Darstellung der ausgewerteten Daten erfolgte ebenfalls mit Hilfe von Excel ${ }^{\circledR}$.

Die aus dem RED $^{\circledR}$-Programm extrahierten Daten wurden in ein Excel ${ }^{\circledR}$-Datenblatt umgewandelt und strukturiert. Die vergleichende Signifikanztestung von boden- und luftgebunden versorgten Kindernotfällen erfolgte durch logistische Regression mittels SPSS Statistics $^{\circledR}$. 


\section{Ergebnisse}

\subsection{Teil I: Analyse der Befragung der Notärzte}

Von den 50 an die aktiven Notärzte des Zentrums Anaesthesiologie, Rettungs- und Intensivmedizin ausgegebenen Fragebögen wurden 43 vollständig und korrekt ausgefüllt (Rücklaufrate $86 \%$ ). Die biometrischen Daten der befragten Notärzte sowie ihre Erfahrung in der präklinischen Notfallmedizin sind in Tabelle 4 dargestellt.

\begin{tabular}{|l|c|c|}
\hline & $\mathrm{n}$ & $\%$ \\
\hline Alter & 9 & 21.9 \\
\hline 25-30 Lebensjahre & 26 & 60.5 \\
\hline 31-40 Lebensjahre & 8 & 18.6 \\
\hline > 40 Lebensjahre & & \\
\hline Geschlecht & 25 & 58.1 \\
\hline Männlich & 18 & 41.9 \\
\hline Weiblich \\
\hline Status \\
\hline In Weiterbildung & 21 & 48.8 \\
\hline Facharzt für Anästhesie & 22 & 51.2 \\
\hline Notfallmedizinische Erfahrung \\
\hline < 2 Jahre & 14 & 32.5 \\
\hline 2-5 Jahre & 19 & 44.2 \\
\hline > 5 Jahre & 10 & 23.3 \\
\hline Erfahrung mit Rettungsmitteln & 30 & 70.0 \\
\hline Nur NEF & 13 & 30.0 \\
\hline NEF und RTH & & \\
\hline
\end{tabular}

Tabelle 4 Persönliche Daten, Ausbildungsstatus und notfallmedizinische Erfahrung der 43 teilnehmenden Notärzte

\subsubsection{Einschätzung der Häufigkeit pädiatrischer Notfälle}

Die persönliche Einschätzung des prozentualen Anteils präklinischer pädiatrischer Notfälle am Gesamteinsatzaufkommen zeigte, dass die Mehrzahl der 43 Befragten ( $n=24 ; 55,8 \%$ ) den Anteil pädiatrischer Notfälle im Bereich 3,1 bis 6,0 \% ansiedeln (Abbildung 1). 


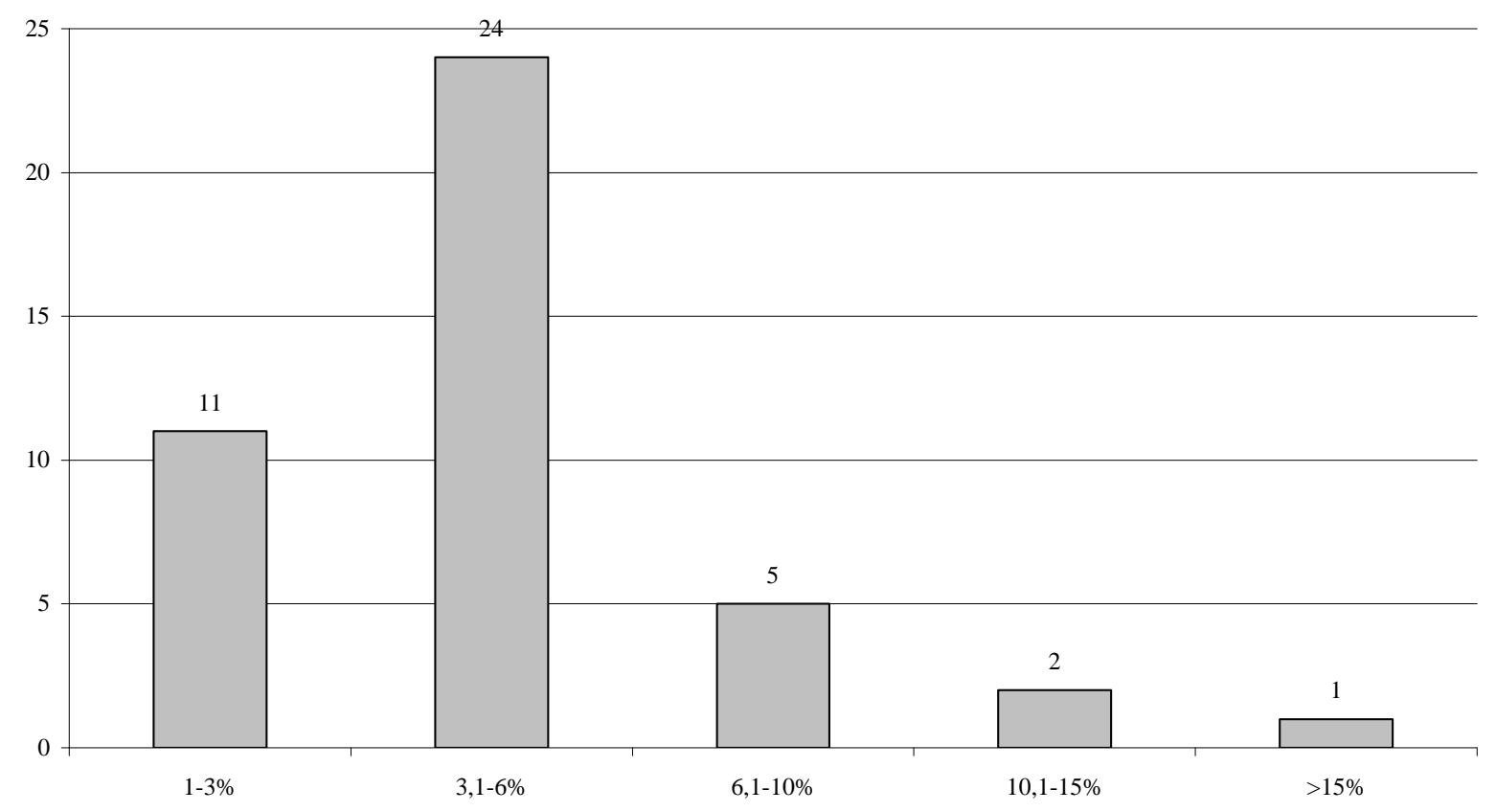

Abbildung 1 Einschätzung des prozentualen Anteils pädiatrischer Notfälle am Gesamteinsatzaufkommen

In der differenzierten Ansicht der Patientengruppe ,Kinder`, unterteilt in die Altersgruppen ,Säuglinge‘ (<1 Lebensjahr), ,Kleinkinder‘ (1-5 Lebensjahre) und ,Schulkinder` (6-14 Lebensjahre) machen Säuglinge nach der Einschätzung der Befragten einen eher geringen Anteil am Patientengut aus, denn 20 Befragte (46,5 \%) schätzten die Häufigkeit präklinischer pädiatrischer Notfälle in dieser Altersgruppe auf einen Anteil von $<10 \%$ am pädiatrischen Gesamteinsatzaufkommen ein. Die geschätzte Häufigkeit von Kleinkindern und Schulkindern wurde dagegen als gleich hoch angenommen $(n=18 ; 41,9 \%$ der Befragten schätzten den Anteil präklinischer pädiatrischer Notfälle in beiden Altersgruppen jeweils im Bereich von 31-50 \% ein) (Abbildung 2). 


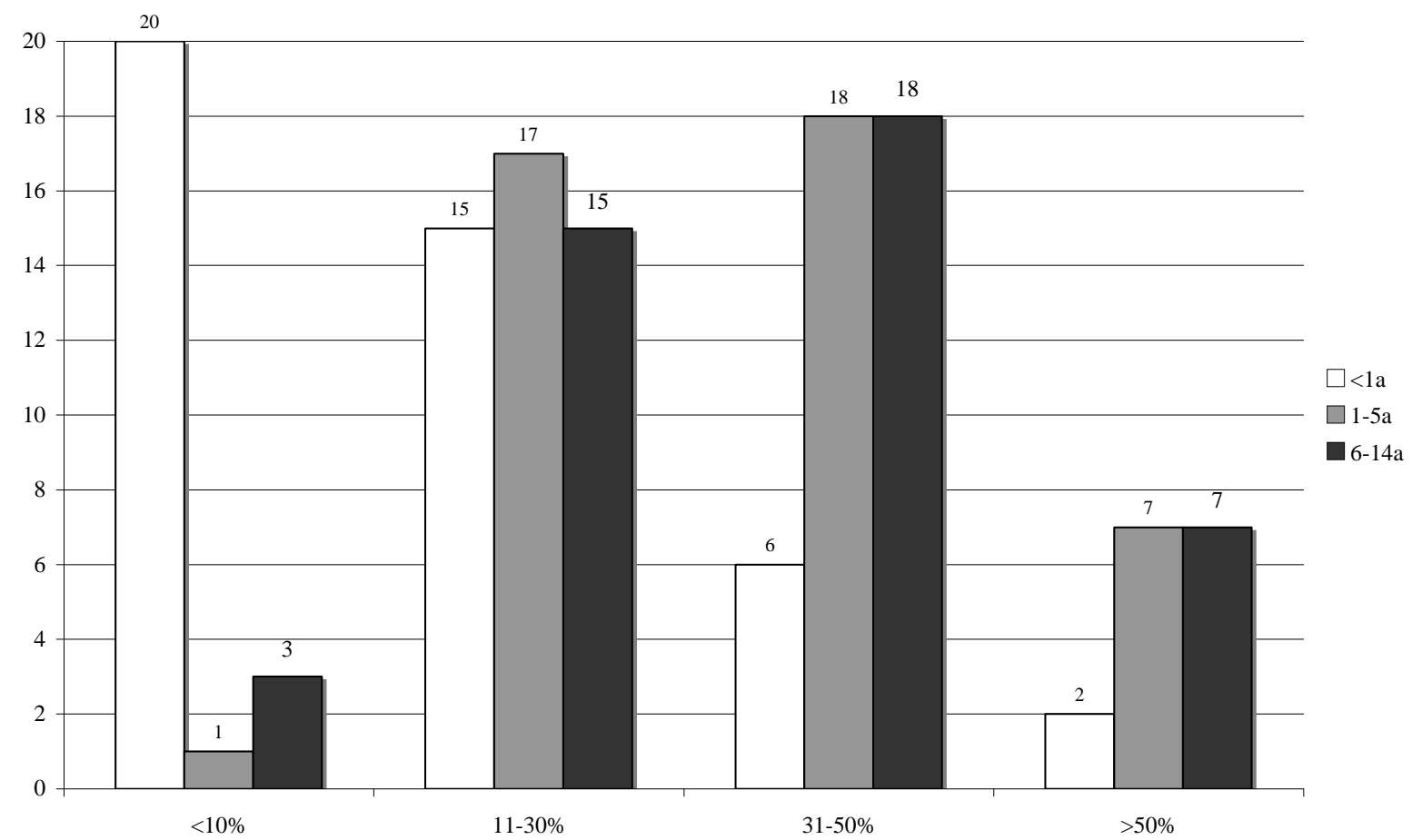

Abbildung 2 Einschätzung des prozentualen Anteils der jeweiligen Altersgruppen am gesamten pädiatrischen Einsatzaufkommen (Säuglinge 0-1 LJ (weiß), Kleinkinder 1-5 LJ (hellgrau), Schulkinder 6-14 LJ (dunkelgrau))

\subsubsection{Einschätzung des Schweregrades präklinischer pädiatrischer Notfälle}

Bei der Einschätzung des Schweregrades präklinischer pädiatrischer Notfälle zeigte sich, dass die überwiegende Mehrzahl der Kindernotfälle in allen drei Altersgruppen als gleichermaßen weniger schwerwiegend (NACA-Score I-III) eingeschätzt $(p<0,01)$ wurde. Auf einer Skala von 1 bis 10 betrugen die Gesamthäufigkeitsmediane für NACA I-III 7,0 in der Altersgruppe der Säuglinge, 6,1 in der Altersgruppe der Kleinkinder und 7,8 in der Altersgruppe der Schulkinder.

\subsubsection{Einschätzung der Häufigkeit von Verdachtsdiagnosen}

Bei der Auswertung der geschätzten Inzidenz der häufigsten Diagnosen in der Patientengruppe Kinder ergaben sich typische Unterschiede zwischen den drei Altersgruppen, insbesondere für die Diagnosen ,plötzlicher Kindstod‘, ,Ertrinken‘, ,Pseudokrupp‘, ,Asthma‘, ,Trauma‘ und ,Schädel-Hirn-Trauma‘ sowie ,Krampfanfall‘. In den Altersgruppen ,Säuglinge‘ und ,Kleinkinder` fanden sich als häufigste Diagnosen ,Krampfanfall‘, „Pseudokrupp‘ und 
,Verbrennung/Verbrühung‘, während in der Altersgruppe ,Schulkinder` als häufigste Diagnosen ,Trauma‘ und ,Schädel-Hirn-Trauma‘ sowie ,Asthma bronchiale‘ eingeschätzt wurden.

Die Anwendung invasiver therapeutischer Maßnahmen bei pädiatrischen Patienten wurde als eher selten eingeschätzt. Am ehesten wurden endotracheale Intubation, intraossäre Punktion sowie kardiopulmonale Reanimation (CPR) als angewendete Interventionen erwartet. Eine Thorakozentese bzw. die Einlage einer Thoraxdrainage und die Defibrillation wurden bei kindlichen Notfällen als Seltenheit eingeschätzt (Abbildung 3). Die Einschätzung erfolgte auf einer Skala von 1 (= sehr selten) bis 10 (= sehr häufig). Die drei häufigsten Diagnosen laut Einschätzung der befragten Notärzte in den entsprechenden Altersgruppen sind in Tabelle 5 dargestellt.

\begin{tabular}{c|l|l|l} 
& $\begin{array}{l}\text { Säuglinge } \\
\text { (<1 Lebensjahr) }\end{array}$ & $\begin{array}{l}\text { Kleinkinder } \\
\text { (1-5 Lebensjahre) }\end{array}$ & $\begin{array}{l}\text { Schulkinder } \\
\text { (6-14 Lebensjahre) }\end{array}$ \\
\hline 1. & $\begin{array}{l}\text { Krampfanfall inklusive Fie- } \\
\text { berkrampf }\end{array}$ & Pseudokrupp & Trauma \\
\hline 2. & Pseudokrupp & $\begin{array}{l}\text { Krampfanfall inklusive Fie- } \\
\text { berkrampf }\end{array}$ & Asthma bronchiale \\
\hline 3. & Verbrennung/Verbrühung & Verbrennung/Verbrühung & Schädel-Hirn-Trauma
\end{tabular}

Tabelle 5: $\quad$ Häufigste Diagnosen in den Altersgruppen 


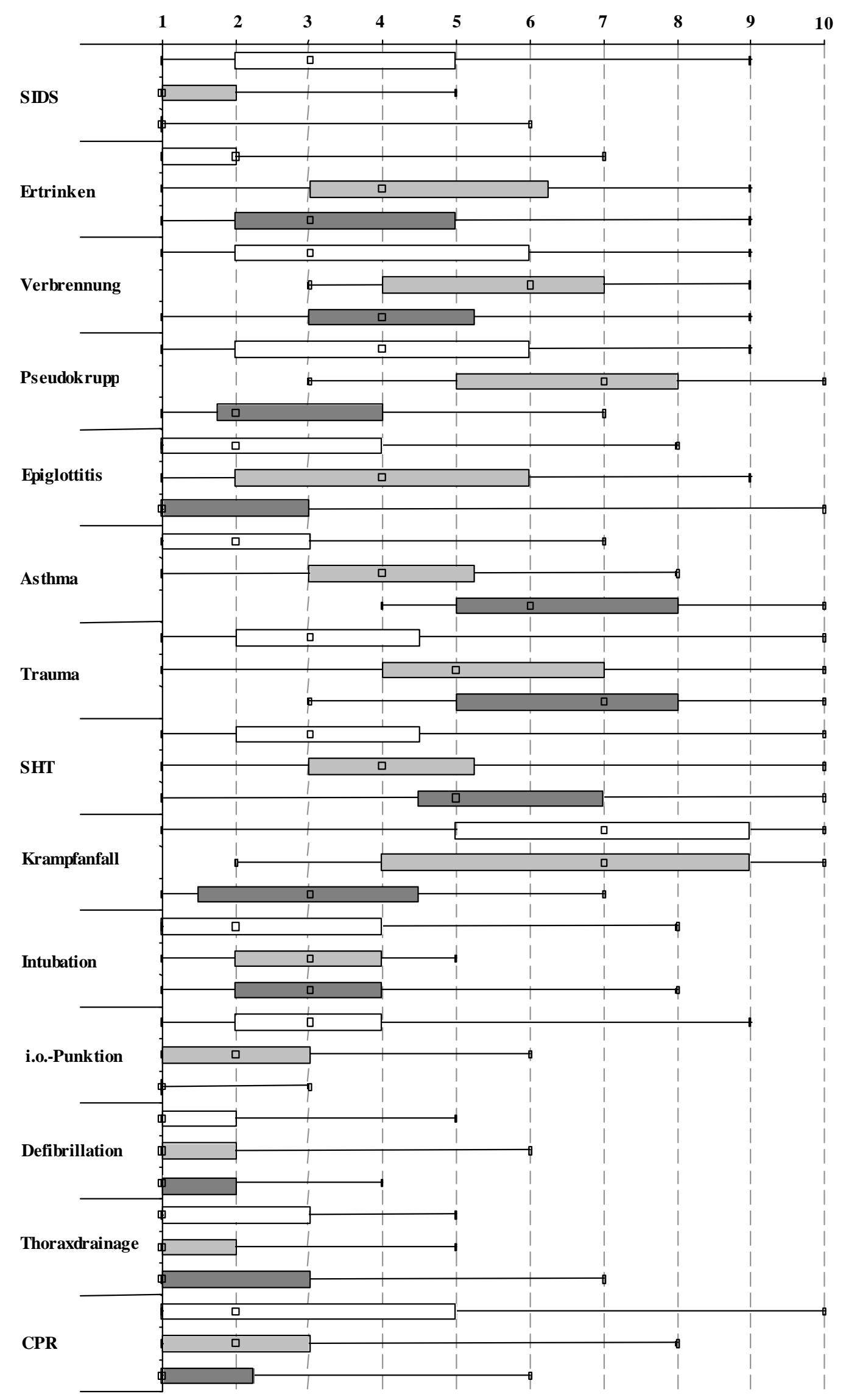

Abbildung 3 Boxplots der geschätzten Inzidenzen von Diagnosen und Interventionen. Skala von $1=$ sehr selten bis 10 = sehr häufig, differenziert für Säuglinge (0-1 LJ, weiße Balken), Kleinkinder (1-5 LJ, hellgrau) und Schulkinder (6-14 LJ, dunkelgrau). 25- bis 75 \%-Intervall, Median und Bereich. 


\subsubsection{Einschätzung des persönlichen Sicherheitsgefühls}

Insgesamt fühlten sich die Notärzte im Umgang mit präklinischen pädiatrischen Notfällen relativ sicher. Dabei stieg das persönliche Sicherheitsempfinden über alle Diagnosen und Interventionen hinweg mit zunehmendem Alter der Kinder. Grundsätzlich fühlten sich die befragten Notärzte im Umgang mit Neugeborenen am unsichersten (Median = 3,0 auf einer Skala von 1 [= völlig unsicher] bis 10 [= völlig sicher]) und bei Schulkindern am sichersten (Median = 8,0) (Abbildung 4).

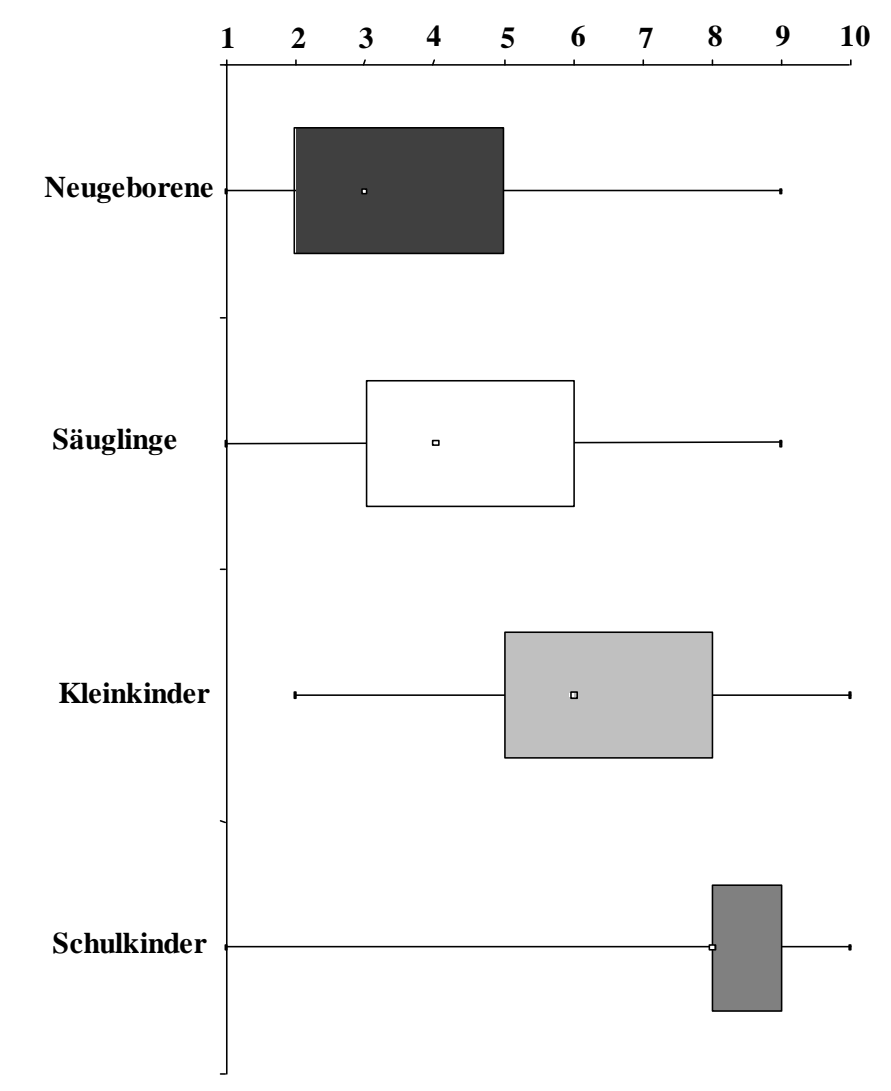

Abbildung 4 Persönliches Sicherheitsempfinden bei präklinischen Kindernotfällen in Abhängigkeit von der Altersgruppe. Skala von 1 = völlig unsicher bis 10 = sehr sicher. Neugeborene (bis 28 Tage), Säuglinge (0-1 LJ), Kleinkinder (1-5 LJ) und Schulkinder (6-14 LJ). 25- bis 75 \%-Intervall, Median und Bereich.

Das persönliche Sicherheitsgefühl im Umgang mit den einzelnen Diagnosen und Interventionen ist in Abbildung 5 dargestellt (auf einer Skala von 1 [= völlig unsicher] bis 10 [= sehr sicher].

Die drei Diagnosen, in denen sich die befragten Notärzte in den entsprechenden Altersgruppen am sichersten fühlten, zeigt Tabelle 6 . 


\begin{tabular}{c|l|l|l} 
& $\begin{array}{l}\text { Säuglinge } \\
\text { (<1 Lebensjahr) }\end{array}$ & $\begin{array}{l}\text { Kleinkinder } \\
\text { (1-5 Lebensjahre) }\end{array}$ & $\begin{array}{l}\text { Schulkinder } \\
\text { (6-14 Lebensjahre) }\end{array}$ \\
\hline 1. & $\begin{array}{l}\text { Krampfanfall inklusive Fie- } \\
\text { berkrampf }\end{array}$ & $\begin{array}{l}\text { Krampfanfall inklusive Fie- } \\
\text { berkrampf }\end{array}$ & Krampfanfall \\
\hline 2. & Pseudokrupp & Pseudokrupp & Asthma bronchiale \\
\hline 3. & Asthma bronchiale & Asthma bronchiale & Trauma
\end{tabular}

Tabelle 6: $\quad$ häufigste geschätzte Diagnosen in den Altersgruppen

Die geschätzte Intervention, bei deren Anwendung sich die befragten Notärzte am sichersten waren, war in allen drei Altersgruppen die Intubation (siehe Abbildung 5). 


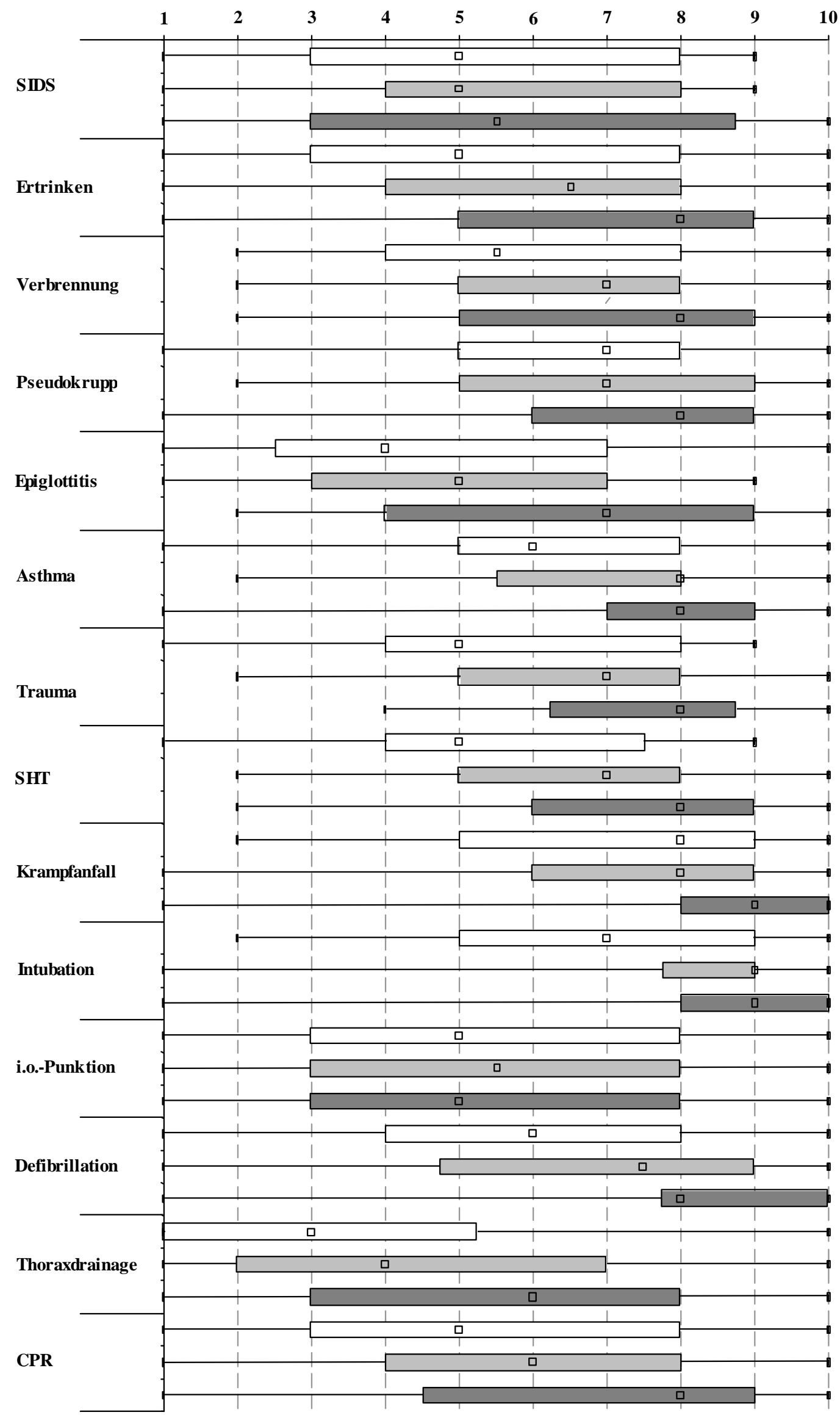

Abbildung 5 Boxplots des persönlichen Sicherheitsempfindens bezüglich Diagnosen und Interventionen. Skala von 1 = sehr unsicher bis $10=$ sehr sicher, differenziert für Säuglinge (0-1 LJ, weiße Balken), Kleinkinder (1-5 LJ, hellgrau) und Schulkinder (6-14 LJ, dunkelgrau).25- bis 75 \%-Intervall, Median und Bereich. 


\subsubsection{Persönliche Erfahrung mit präklinischen Kindernotfällen}

Zur persönlichen Erfahrung mit präklinischen Kindernotfällen wurden folgende Angaben gemacht: 17 von 43 Antwortenden hatten bereits pädiatrische Traumata versorgt (zehn mit Schädel-Hirn-Trauma, vier mit Polytrauma). Elf Befragte hatten Erfahrung im Umgang mit Pseudokrupp gesammelt, neun mit Verbrennungen bzw. Verbrühungen, sieben hatten Kinder nach Ertrinken behandelt, drei Kinder mit Anaphylaxie und jeweils zwei Befragte hatten Kinder mit akuter Atemnot, Epiglottitis und Atem-Kreislauf-Stillstand versorgt (Tabelle 7).

\begin{tabular}{l|l} 
Pädiatrischer Notfall & $n$ \\
\hline Trauma & 17 \\
Pseudokrupp & 11 \\
SHT & 10 \\
Verbrennung/Verbrühung & 9 \\
Asthma bronchiale & 8 \\
Ertrinkungsunfall & 7 \\
Verkehrsunfall (Polytrauma) & 4 \\
Anaphylaxie & 3 \\
Atemnot & 2 \\
Epiglottitis & 2 \\
Reanimation & 2 \\
APGAR 0 & 1 \\
Akute Appendizitis & 1 \\
Kreislaufstillstand & 1 \\
Magen-Darm-Infekt & 1 \\
Stromunfall & 1 \\
A &
\end{tabular}

Tabelle $7 \quad$ Angabe von bereits behandelten pädiatrischen Notfällen

Fünfzehn Notärzte hatten zum Zeitpunkt der Befragung bereits mindestens einmal spezielle weiterführende Kurse zur pädiatrischen Notfallversorgung (EPLS, PALS, Kindersimulationskurs, Notarzt-Refresherkurs o. ä.) besucht. Sieben Befragte machten zu ihrem Fortbildungsstand keine Aussage und 21 Befragungsteilnehmer hatten zum Zeitpunkt der Befragung keinen speziellen Weiterbildungskurs für pädiatrische Notfälle besucht. 
Als subjektiv empfundene Ausbildungsdefizite nannten die Befragten besonders häufig die kardiopulmonale Reanimation von Kindern, die Versorgung (schwer) verletzter Kinder sowie der Umgang mit der Diagnose plötzlicher Kindstod (SIDS) (Tabelle 8).

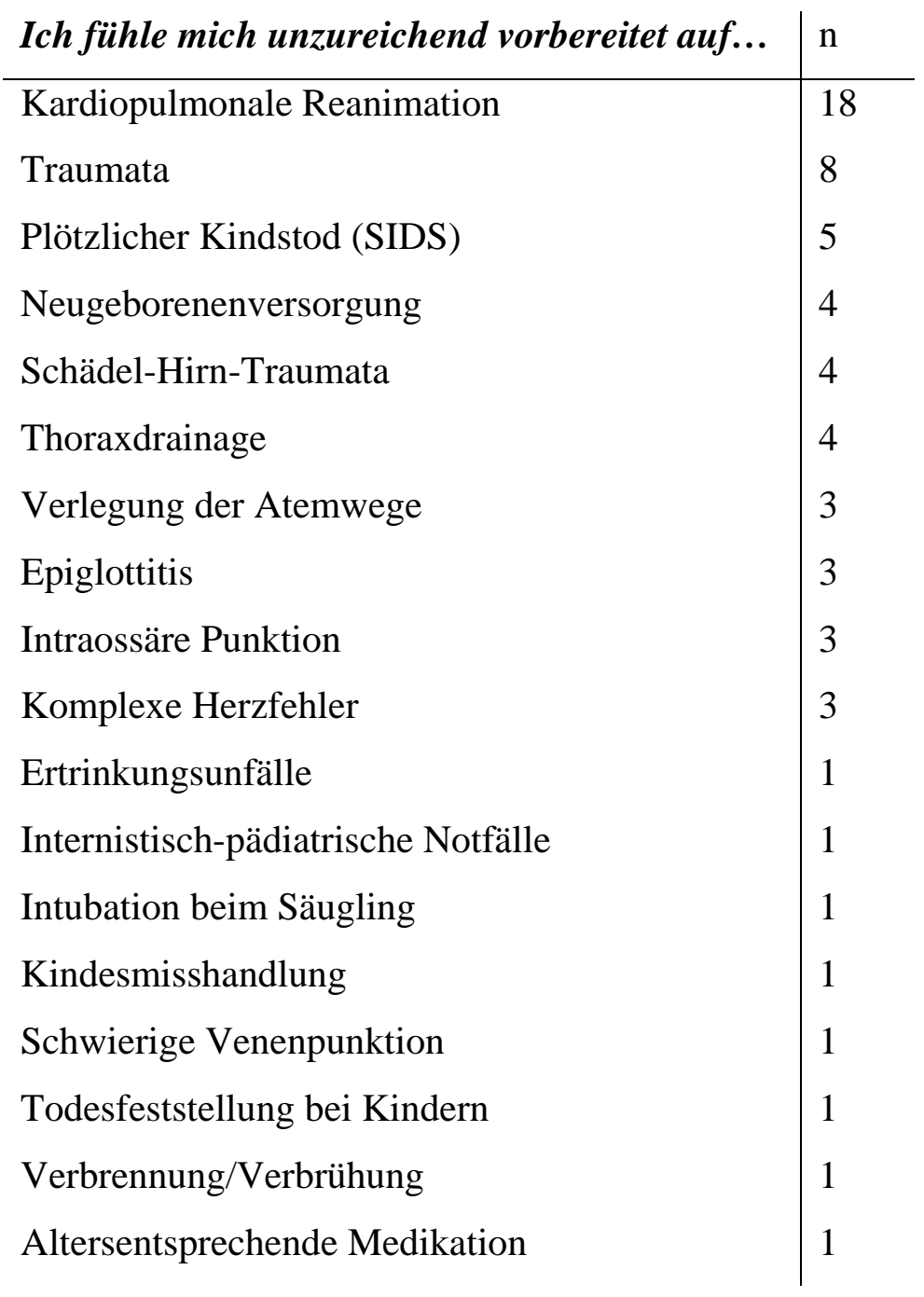

Tabelle $8 \quad$ Persönlich empfundene Ausbildungs- und Erfahrungsdefizite in der Kindernotfallmedizin

Bei der Frage nach Vorschlägen zur Verringerung der von den Befragten empfundenen Ausbildungs- und Erfahrungsdefizite schlug die Mehrzahl der Notärzte ein regelmäßiges Training am Kindersimulator vor. Acht Befragte schlugen Praktika in der Neonatologie und Kinderintensivmedizin vor, sieben nannten die Einführung spezieller kindernotfallmedizinischer Kursangebote im Rahmen einer regelhaften Notarzt-Rezertifizierung und vier votierten für häufigere Einsätze in der Kinderanästhesie. Einzelnennungen waren die Zuhilfenahme von Videodokumentationen realer Einsätze, eine intensivierte Supervision, die Ernennung lokaler Ausbildungsbeauftragter für die Kindernotfallmedizin, die Etablierung eines Kinder-NEF auch in dezentralen Rettungsdienstbereichen, klarere Definitionen der Notarztanforderungen 
sowie eine allgemeine Sensibilisierung für die Problematik von präklinischen pädiatrischen Notfällen (Tabelle 9).

\begin{tabular}{l|l}
\hline Verbesserungsvorschläge & $n$ \\
\hline Simulationstraining & 24 \\
(regelmäßiges Simulationstraining, mindestens einmal pro Jahr) & $(6)$ \\
Praktika in der Kinderintensivmedizin bzw. entsprechenden Einrichtungen der Früh- und & 8 \\
Neugeborenenversorgung & \\
Kursangebote zur Re-Zertifizierung als Notarzt & 7 \\
Routine im OP mit intensivierter Rotation in die Kinderchirurgie und Erhöhung der An- \\
zahl für Pflichtintubationen bei Kindern in der anästhesiologischen Facharztausbildung \\
Videodokumentation realer Einsätze mit anschließender Auswertung & \\
Reale Einsätze mit fachkundiger Supervision & 1 \\
Einrichtung eines Ausbildungsbeauftragten für Kinderintensivmedizin in der jeweiligen & 1 \\
Klinik & 1 \\
Etablierung eines spezialisierten Kinder-NEF in Göttingen & \\
Klare Definition der Notarztanforderungen & 1 \\
Sensibilisierung der Ärzte und Rettungsdienstmitarbeiter bezüglich der Problematik & 1 \\
„Kindernotfall“ & 1 \\
\hline
\end{tabular}

Tabelle 9 Verbesserungsvorschläge für die kindernotfallmedizinische Aus-, Weiter- und Fortbildung 
Bei der Frage nach Notfällen und notfallmedizinischen Interventionen, welche die Notärzte gerne am Kindersimulator trainieren würden, wurden am häufigsten die kardiopulmonale Reanimation, das Atemwegs- und Traumamanagement sowie das Legen von intraossären Kanülen bzw. von Gefäßzugängen genannt (Tabelle 10).

\begin{tabular}{l|l}
\hline Notfallsituation & $n$ \\
\hline Kardiopulmonale Reanimation & 21 \\
Atemwegsmanagement & 11 \\
Traumaversorgung & 10 \\
Intraossäre Punktion & 9 \\
Intravenöse Punktion & 4 \\
Epiglottitis & 3 \\
Ertrinkungsunfall & 3 \\
Pseudokrupp & 3 \\
Asthma bronchiale & 2 \\
Früh- \& Neugeborenenversorgung & 2 \\
Koniotomie & 2 \\
Schädel-Hirn-Traumata & 2 \\
Plötzlicher Kindstod (SIDS) & 1 \\
Hypovolämie/Schock & 1 \\
Verdacht auf Kindesmisshandlung & 1 \\
Komplexe Herzfehler & 1 \\
Krampfanfall & 1 \\
Laryngospasmus & 1 \\
Pneumothorax & 1 \\
Unterkühlung & 1 \\
Verbrennung/Verbrühung & 1 \\
\hline
\end{tabular}

Tabelle $10 \quad$ Kindliche Notfälle und Interventionen, welche die Notärzte bevorzugt am Kindersimulator trainieren würden. 


\subsection{Teil II: Ergebnisse der Analyse der Rettungsdiensteinsatzdokumentation ${ }^{\circledR}$ $\left(\right.$ RED $\left.^{\circledR}\right)$}

Über den Studienzeitraum von neun Jahren wurden 2.271 präklinische pädiatrische Fälle im RED $^{\circledR}$ identifiziert. Davon konnten 319 Fälle (14,0 \%) der Altersgruppe ,Säuglinge‘ (<1 Lebensjahr), 957 Fälle (42,1 \%) der Altersgruppe ,Kleinkinder‘ (1-5 Lebensjahre) und 995 Fälle (43,8 \%) der Altersgruppe ,Schulkinder` (6-14 Lebensjahre) zugeordnet werden (Abbildung 6). Bei einem durchschnittlichen jährlichen Gesamteinsatzaufkommen von 4.027 präklinisch versorgten Notfällen beträgt die Anzahl der pädiatrischen Notfälle im Untersuchungszeitraum im Mittel 252, was einem prozentualen Anteil von 6,3 \% am notärztlichen Gesamteinsatzaufkommen im Rettungsdienstbereich Göttingen entspricht.

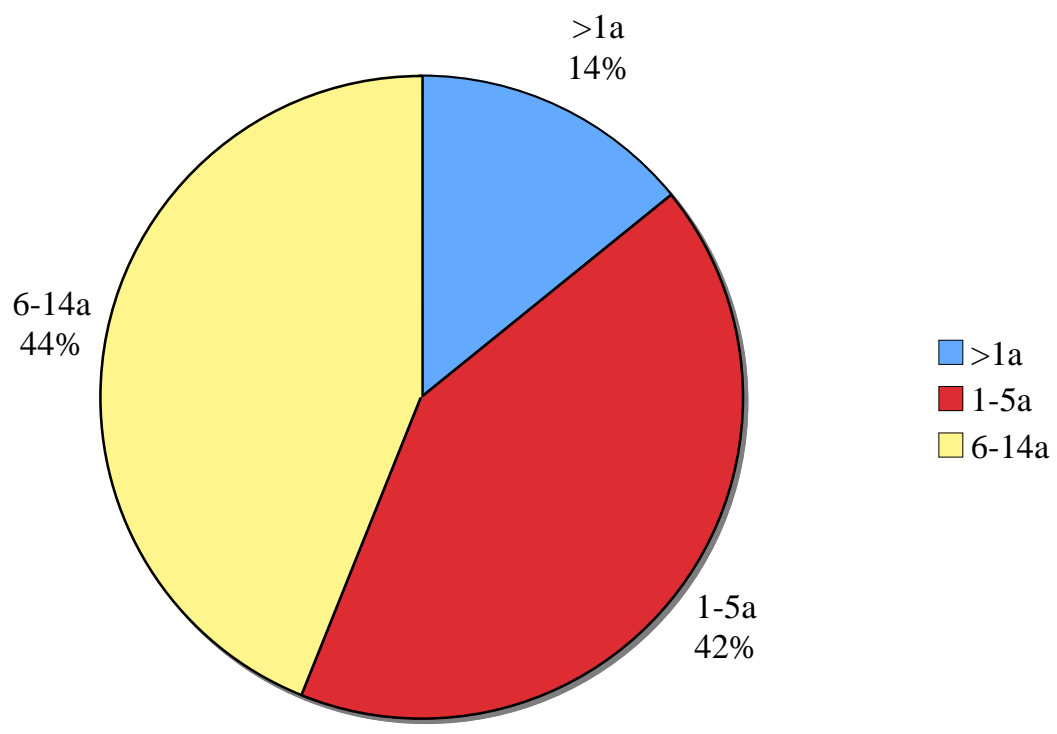

Abbildung 6 Anteil pädiatrischer Notfälle nach RED ${ }^{\circledR}$ entsprechend den Altersgruppen blau $=<1$ LJ (Säuglinge), gelb = 1-5 LJ (Kleinkinder), rot = 6-14 LJ (Schulkinder)

\subsubsection{Differenzierung nach dem Rettungsmittel}

Differenziert nach dem Rettungsmittel betrug der Anteil pädiatrischer Notfälle am Gesamteinsatzaufkommen im bodengebundenen Rettungsdienst 5,2 \% und im luftgebundenen Rettungsdienst $8,5 \%$. 
988 pädiatrische Notfälle (43,5 \%) wurden durch luftgebunden-tätige Rettungsteams versorgt. In 1.283 Fällen (56,5\%) wurde die Versorgung durch bodengebundene Teams (NEF) durchgeführt (Abbildung 7).

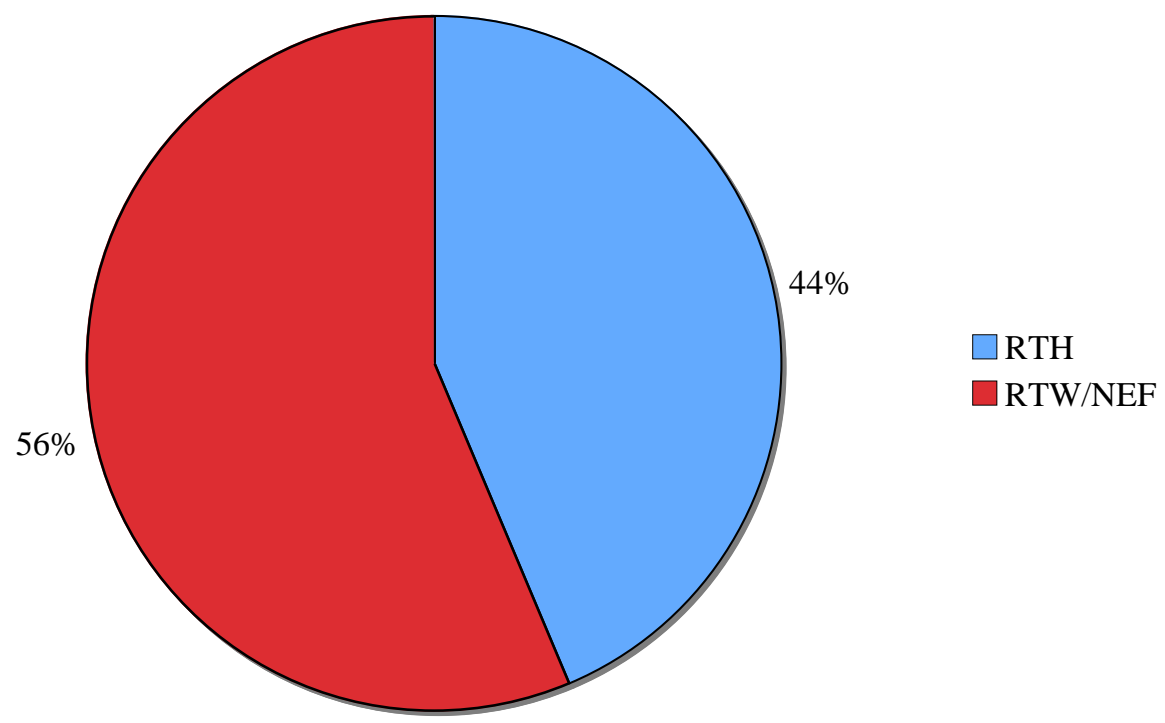

Abbildung $7 \quad$ Anteil pädiatrischer Notfälle versorgt durch luftgebundene (blau) und bodengebundene (rot) Rettungsteams

Mit zunehmendem Lebensalter wurde die Versorgung pädiatrischer Notfallpatienten durch luftgebundene Rettungsteams häufiger: 37,3 \% der Säuglinge, 42,3 \% der Kleinkinder und 46,6 \% der Schulkinder wurden durch luftgebundene Rettungsteams versorgt. Einen Überblick über die Eigenschaften präklinischer Kindernotfälle in Göttingen im untersuchten Zeitraum gibt Tabelle 11.

\subsubsection{Schweregrad der Notfälle}

Präklinische Kindernotfälle waren überwiegend geringeren Schweregrades. 72,7 \% der Kindernotfälle waren vom Schweregrad NACA I-III, während nur 27,3 \% der Kindernotfälle einem höheren Schweregrad (im Bereich NACA IV-VII) zuzuordnen waren. Gleichermaßen verhielt es sich bei den Notfällen, in denen ein Trauma beim pädiatrischen Patienten diagnostiziert wurde. Hier befand sich ebenfalls der Großteil der Traumata im weniger schwerwiegenden Bereich (NACA I-III), siehe Abbildung 8. 


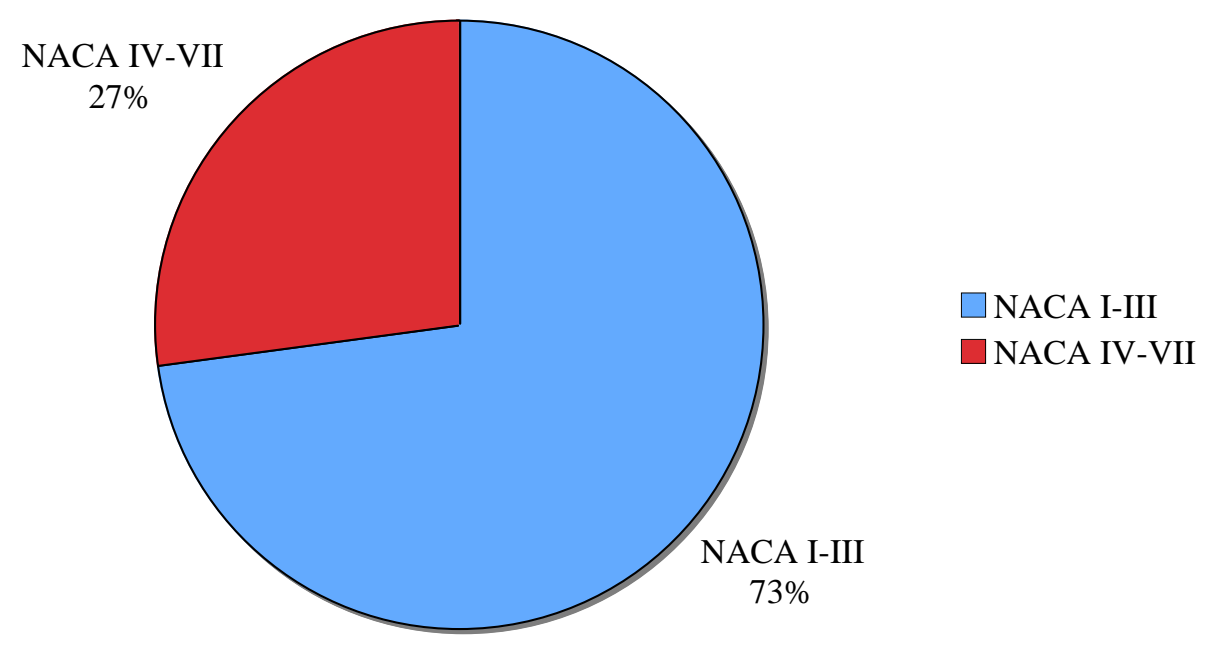

(a)

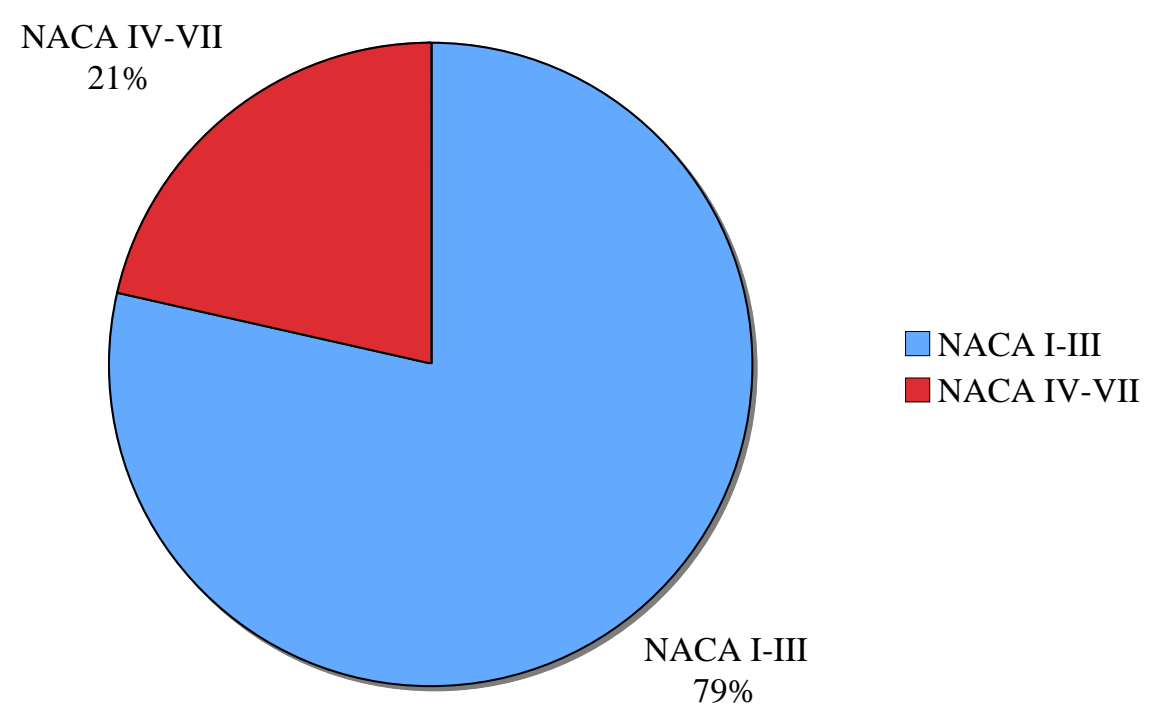

(b)

Abbildung 8 Anteil NACA I-III, NACA IV-VII nach RED ${ }^{\circledR}$ insgesamt (a), Anteil Trauma-NACA I-III, NACA IV-VII nach RED $^{\circledR}$ (b) blau = NACA I-III, rot = NACA IV-VII 
In den Altersgruppen der Säuglinge und Kleinkinder wiesen die untersuchten Notfällen im Vergleich höhere NACA-Scores auf als in der Altersgruppe der Schulkinder (Abbildung 9).

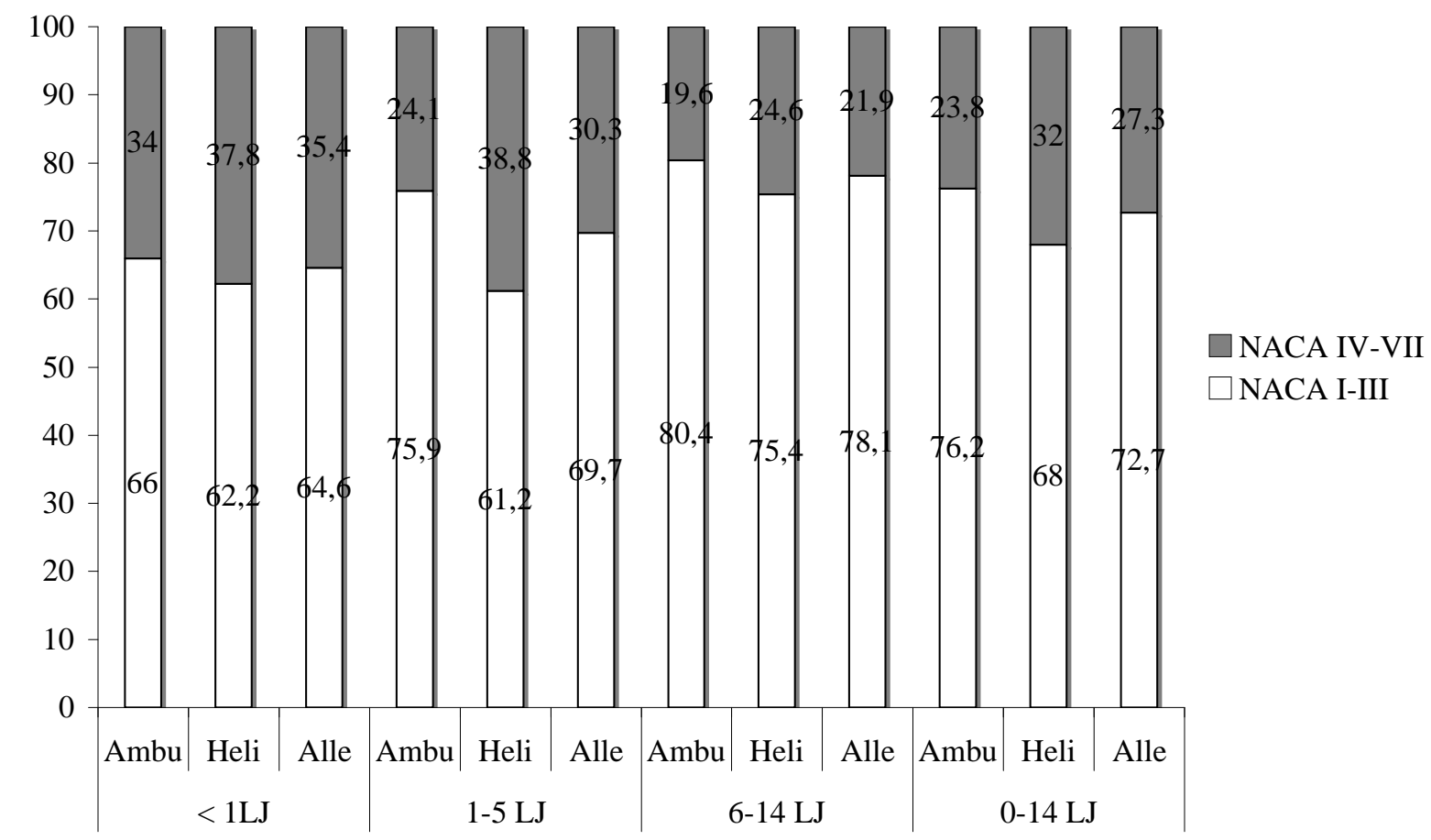

Abbildung 9 NACA-Scores für präklinische Kindernotfälle und Notfälle Erwachsener, differenziert nach Altersgruppen (Säuglinge (<1 LJ), Kleinkinder (1-5 LJ) und Schulkinder (6-14 LJ) und Transportmittel

\subsubsection{Transportziel}

Das Transportziel war in 1.823 Fällen (80,3 \%) die Universitätsmedizin Göttingen. In 162 Fällen (7,1\%) wurden die Kinder primär in ein anderes Krankenhaus transportiert. Bei 286 pädiatrischen Notfällen (12,6 \%) wurde ein Transport als unnötig erachtet. Diese Kinder wurden zur weiteren ambulanten Versorgung durch Allgemeinmediziner oder Pädiater zunächst in der elterlichen Obhut belassen (Abbildung 10). 


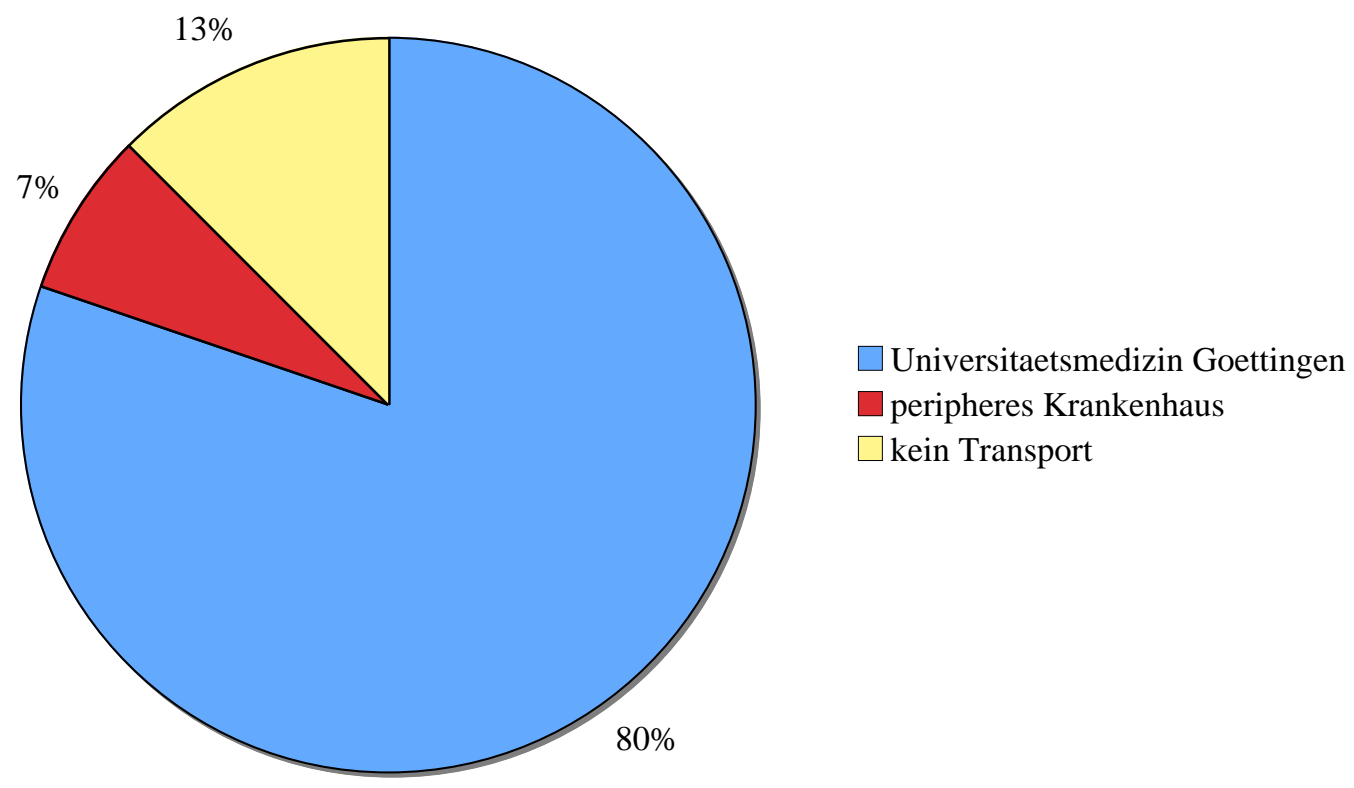

Abbildung 10 Transportziel

239 (74,9 \%) der behandelten Säuglinge wurden in die Universitätsmedizin Göttingen verbracht, 20 Säuglinge (6,3 \%) wurden in andere Krankenhäuser eingeliefert. 60 (18,8 \%) der von den Notärzten der Universitätsmedizin behandelten Säuglinge wurden nicht transportiert. In der Altersgruppe der Kleinkinder wurden 808 der Patienten (84,4\%) in die Universitätsmedizin Göttingen, 27 (2,8 \%) in andere Krankenhäuser transportiert, während 122 der Kinder in dieser Altersgruppe (12,8 \%) nicht transportiert wurden. Bei den Schulkindern zeigte sich eine geringere Zahl von Patienten, die nicht transportiert wurden. Nur 104 Schulkinder (10,4 \%) wurden nicht transportiert, während 776 (78 \%) in die Universitätsmedizin Göttingen und 115 (11,6 \%) in andere Krankenhäuser verbracht wurden.

\subsubsection{Diagnosen}

Bei Säuglingen waren respiratorische Störungen (SIDS, Ertrinken, Pseudokrupp, Epiglottitis, Asthma bronchiale, Aspiration und andere respiratorische Störungen) mit einem Anteil von 34,5 \% die führenden Notfalldiagnosen, gefolgt von Krampfanfällen/Fieberkrämpfen (17,2 \%) und Traumata (16,0 \%). Für Kleinkinder ergaben sich als vorrangig zum Notruf führende Diagnosen Krampfanfälle/Fieberkrämpfe (43,2 \%), respiratorische Störungen (21,8 \%) sowie Traumata (19,5\%). In der Altersgruppe ,Schulkinder‘ war die Diagnose ,Trauma‘, die 
bei weitem häufigste Einweisungsdiagnose (44,4\%). Neurologische (Krampfanfälle) und respiratorische Störungen nahmen mit 16,0 \% und 15,0 \% Platz zwei und drei in dieser Altersgruppe ein (Abbildung 11, Tabelle 11).

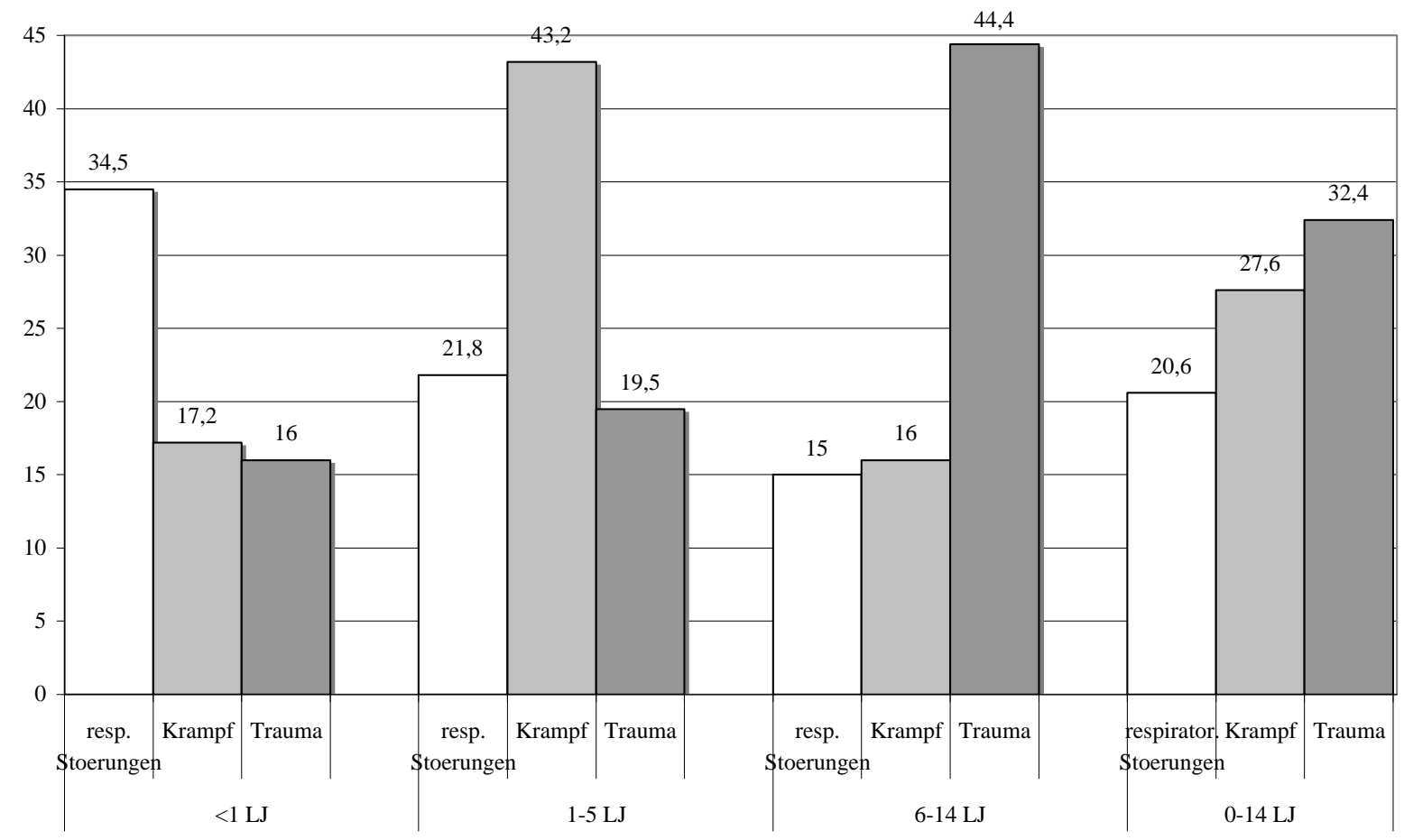

Abbildung 11 Die häufigsten Diagnosen für die Altersgruppen Säuglinge (< 1 LJ), Kleinkinder (1-5 LJ) und Schulkinder (6-14 LJ) laut RED ${ }^{\circledR}$

\subsubsection{Interventionen}

Invasive Prozeduren bei Kindern wurden selten angewendet. Mit Ausnahme der hier nicht erfassten periphervenösen Punktion war die häufigste Intervention die endotracheale Intubation. Diese Intervention wurde bei Säuglingen in 24 (7,5 \%), bei Kleinkindern in 31 (3,2 \%) und bei Schulkindern in 41 (4,1 \%) Fällen angewendet. Fast ebenso häufig wurde von den Notärzten die kardiopulmonale Reanimation mit Anteilen von 7,5 \% $(n=24)$ bei Säuglingen, $1,9 \%(n=18)$ bei Kleinkindern und 1,0 \% $(n=10)$ bei Schulkindern durchgeführt. Die intraossäre Punktion war bei neun Säuglingen (2,8 \%) und sechs Kleinkindern (0,6 \%) erforderlich. Thoraxdrainage/Pleurapunktion sowie Defibrillation wurden im Untersuchungszeitraum kaum angewendet $(<1,0 \%)$. 


\subsubsection{Diagnosen und Interventionen in Abhängigkeit vom Rettungsmittel}

Im Bereich der luftgebundenen Notfallversorgung fand sich insgesamt ein wesentlich höheres Aufkommen von Diagnosen wie Ertrinken (80,0 \%), Kopfverletzungen (73,9 \%), Verbrennungen/Verbrühungen (38,6 \%) und Notfällen mit SIDS (60,0\%). Ebenso waren die Inzidenzen für invasive Prozeduren wie Intubation (78,1 \%), intraossäre Punktion (66,7 \%) und Defibrillation (83,3\%) sowie Thoraxdrainage (100\%) und kardiopulmonale Reanimation höher im Vergleich zu ihrem Aufkommen in der bodengebundenen Rettung. Im Gegensatz dazu wurden 75,6 \% aller respiratorischen Störungen durch bodengebundene Rettungsteams versorgt (Tabelle 11).

Die Wahrscheinlichkeit der Anwesenheit eines luftgebunden-tätigen Notarztes am Einsatzort zeigte keine signifikante Korrelation mit dem Alter beziehungsweise der Altersgruppen des pädiatrischen Notfallpatienten oder der Verdachtsdiagnose Krampfanfall. Es zeigte sich jedoch eine erhöhte Wahrscheinlichkeit der Beteiligung eines luftgebunden-tätigen Notarztes bei der Diagnose ,Trauma‘ ( $p<0,001)$, während die Diagnose ,respiratorische Störung‘ eine erhöhte Wahrscheinlichkeit für die Anwesenheit eines bodengebunden-tätigen Notarztes aufzeigte $(p=0,001)$ (siehe Tabelle 11). 


\begin{tabular}{|c|c|c|c|c|c|c|c|c|c|c|c|c|c|c|c|c|c|c|c|c|}
\hline & \multicolumn{5}{|c|}{$0-1 \mathrm{LJ}$} & \multicolumn{5}{|c|}{ 1-5 LJ } & \multicolumn{5}{|c|}{ 6-14 LJ } & \multicolumn{5}{|c|}{ 0-14 LJ } \\
\hline & Total & $\begin{array}{c}\text { \% Anteil } \\
\text { Altersgruppe }\end{array}$ & Amb & Heli & $\begin{array}{c}\% \\
\text { Heli }\end{array}$ & Total & $\begin{array}{c}\text { \% Anteil } \\
\text { Altersgruppe }\end{array}$ & Amb & Heli & $\begin{array}{c}\% \\
\text { Heli }\end{array}$ & Total & $\begin{array}{c}\% \text { Anteil } \\
\text { Altersgruppe }\end{array}$ & Amb & Heli & $\begin{array}{c}\% \\
\text { Heli }\end{array}$ & Total & $\begin{array}{c}\% \\
\text { Anteil } \\
\text { Kinder }\end{array}$ & Amb & Heli & $\begin{array}{c}\% \\
\text { Heli }\end{array}$ \\
\hline Alle Diagnosen & 319 & 100 & 200 & 119 & 37.3 & 957 & 100 & 552 & 405 & 42.3 & 995 & 100 & 531 & 464 & 46.6 & 2271 & 100 & 1283 & 988 & 43.5 \\
\hline $\begin{array}{l}\text { Respiratorische Stö- } \\
\text { rungen (total) }\end{array}$ & 110 & 34.5 & 75 & 35 & 31.8 & 209 & 21.8 & 165 & 44 & 21.1 & 149 & 15.0 & 114 & 35 & 23.5 & 468 & 20.6 & 354 & 114 & 24.4 \\
\hline SIDS & 15 & 4.7 & 6 & 9 & 60.0 & 0 & 0.0 & 0 & 0 & $\mathrm{n} / \mathrm{a}$ & 0 & 0.0 & 0 & 0 & $\mathrm{n} / \mathrm{a}$ & 15 & 0.4 & 6 & 9 & 60.0 \\
\hline Ertrinken & 0 & 0.0 & 0 & 0 & $\mathrm{n} / \mathrm{a}$ & 17 & 1.8 & 3 & 14 & 82.4 & 3 & 0.3 & 1 & 2 & 66.7 & 20 & 0.9 & 4 & 16 & 80.0 \\
\hline Pseudokrupp & 13 & 4.1 & 11 & 2 & 15.4 & 104 & 10.9 & 98 & 6 & 5.8 & 36 & 3.6 & 34 & 2 & 5.6 & 153 & 6.7 & 143 & 10 & 6.5 \\
\hline Epiglottitis & 0 & 0.0 & 0 & 0 & $\mathrm{n} / \mathrm{a}$ & 1 & 0.1 & 1 & 0 & 0.0 & 0 & 0.0 & 0 & 0 & $\mathrm{n} / \mathrm{a}$ & 1 & 0.04 & 1 & 0 & 0.0 \\
\hline Asthma & 2 & 0.6 & 2 & 0 & 0.0 & 12 & 1.3 & 11 & 1 & 9.1 & 28 & 2.8 & 22 & 6 & 27.3 & 42 & 1.8 & 35 & 7 & 16.7 \\
\hline Aspiration/FBAO & 37 & 11.6 & 24 & 13 & 35.1 & 24 & 2.5 & 14 & 10 & 41.7 & 16 & 1.6 & 6 & 10 & 62.5 & 77 & 3.4 & 44 & 33 & 42.9 \\
\hline andere respir. & 43 & 13.5 & 32 & 11 & 25.6 & 51 & 5.3 & 38 & 13 & 25.5 & 66 & 6.6 & 51 & 15 & 22.7 & 160 & 7.0 & 121 & 39 & 24.4 \\
\hline Trauma (total) & 51 & 16.0 & 19 & 32 & 62.7 & 187 & 19.5 & 70 & 117 & 62.6 & 482 & 44.4 & 191 & 291 & 60.4 & 720 & 32.4 & 280 & 440 & 61.1 \\
\hline Kopfverletzung & 11 & 3.4 & 5 & 6 & 54.5 & 55 & 5.7 & 14 & 41 & 74.5 & 87 & 8.7 & 21 & 66 & 75.9 & 153 & 6.7 & 40 & 113 & 73.9 \\
\hline Verbrenung/Verbrühung & 8 & 2.5 & 1 & 7 & 88.0 & 36 & 3.8 & 15 & 21 & 58.3 & 12 & 1.2 & 7 & 5 & 41.7 & 56 & 2.5 & 23 & 33 & 58.9 \\
\hline andere Traumata & 32 & 10.0 & 13 & 19 & 59.4 & 96 & 10.0 & 41 & 55 & 57.3 & 383 & 38.5 & 163 & 220 & 56.6 & 511 & 22.5 & 217 & 294 & 57.5 \\
\hline Krampfanfälle (total) & 55 & 17.2 & 30 & 25 & 45.5 & 413 & 43.2 & 235 & 178 & 43.1 & 159 & 16.0 & 98 & 61 & 38.4 & 627 & 27.6 & 363 & 264 & 42.1 \\
\hline Fieberkrampf & 31 & 9.7 & 16 & 15 & 48.4 & 307 & 32.1 & 175 & 132 & 43.0 & 19 & 1.9 & 10 & 9 & 47.4 & 357 & 15.7 & 201 & 156 & 43.7 \\
\hline Andere Krampfanfälle & 24 & 7.5 & 14 & 10 & 41.7 & 106 & 11.1 & 60 & 46 & 43.4 & 140 & 14.1 & 88 & 52 & 37.1 & 270 & 11.9 & 162 & 108 & 40.0 \\
\hline Andere Diagnosen & 103 & 32.3 & 76 & 27 & 26.2 & 148 & 15.5 & 82 & 66 & 44.6 & 205 & 20.6 & 128 & 77 & 37.6 & 456 & 20.1 & 286 & 170 & 36.6 \\
\hline \multicolumn{21}{|l|}{ Interventionen } \\
\hline Intubation & 24 & 7.5 & 8 & 16 & 66.7 & 31 & 3.2 & 5 & 26 & 83.9 & 41 & 4.1 & 8 & 33 & 80.5 & 96 & 4.2 & 21 & 75 & 78.1 \\
\hline Intraossäre Punktion & 9 & 2.8 & 4 & 5 & 55.6 & 6 & 0.6 & 1 & 5 & 83.3 & 0 & 0.0 & 0 & 0 & $\mathrm{n} / \mathrm{a}$ & 15 & 0.7 & 5 & 10 & 66.7 \\
\hline Defibrillation & 3 & 0.9 & 1 & 2 & 66.7 & 3 & 0.3 & 0 & 3 & 100 & 0 & 0.0 & 0 & 0 & $\mathrm{n} / \mathrm{a}$ & 6 & 0.3 & 1 & 5 & 83.3 \\
\hline Throaxdrainage & 0 & 0.0 & 0 & 0 & $\mathrm{n} / \mathrm{a}$ & 2 & 0.2 & 0 & 2 & 100 & 2 & 0.2 & 0 & 2 & 100 & 4 & 0.2 & 0 & 4 & 100 \\
\hline CPR & 24 & 7.5 & 11 & 13 & 54.2 & 18 & 1.9 & 4 & 14 & 77.8 & 10 & 1.0 & 3 & 7 & 70.0 & 52 & 2.3 & 18 & 34 & 65.4 \\
\hline
\end{tabular}

Tabelle 11 Eigenschaften präklinischer Kindernotfälle dokumentiert in der Rettungsdiensteinsatzdokumentation Göttingen (RED ${ }^{\circledR}$ ). 


\section{Diskussion}

Die wichtigsten Ergebnisse dieser Untersuchung lassen sich wie folgt zusammenfassen:

1. Die Einschätzung und Wahrnehmung präklinischer Kindernotfälle durch die befragten Notärzte des Zentrums Anaesthesiologie, Rettungs- und Intensivmedizin der Universitätsmedizin Göttingen erfolgte sehr differenziert.

2. Das persönliche Sicherheitsempfinden stieg mit dem Alter der Kinder und der klinischen Erfahrung bezüglich der Diagnosen und Interventionen.

3. Die größten Erfahrungsdefizite sahen die Befragten in der kardiopulmonalen Reanimation und der Traumaversorgung von Kindern.

4. Zur Optimierung der Vorbereitung auf präklinische pädiatrische Notfälle wurden vor allem das Training am Kindersimulator und Praktika in der Kinderintensivmedizin und -anästhesie favorisiert.

5. Der Anteil pädiatrischer Notfälle lag im Einsatzbereich der Göttinger Notärzte bei 6,3 \%.

6. Die häufigsten Notfalldiagnosen waren Traumata (vor allem Schulkinder), Krampfanfälle (vor allem Kleinkinder) und respiratorische Störungen (vor allem Säuglinge).

7. Invasive Interventionen wurden, mit Ausnahme der periphervenösen Kanülierung, insgesamt eher selten durchgeführt, wobei endotracheale Intubation, kardiopulmonale Reanimation und intraossäre Punktion am häufigsten vorkamen.

8. Der Großteil der pädiatrischen Notfälle war von geringerem Schweregrad (NACA-Scores I bis III).

9. Für luftgebunden-tätige Notärzte besteht eine größere Wahrscheinlichkeit zur Versorgung von Kindernotfällen allgemein sowie zur Versorgung von kränkeren Kindern, bei denen der Einsatz invasiver Interventionen häufiger erforderlich ist.

\subsection{Problematik des kindlichen Notfalles}

Auf einen präklinischen pädiatrischen Notfall fühlen sich Notärzte oft unzureichend vorbereitet (Bartels 2001, Knapp 1995, Zink et al. 2004). Bei der Behandlung von Kindernotfällen ergeben sich folgende besondere Schwierigkeiten:

- die von erwachsenen Patienten abweichende Pathophysiologie, die eine Situationseinschätzung für den Notarzt schwer machen kann;

- die vom Erwachsenen abweichende Pharmakokinetik und -dynamik, die leicht zu Überoder Unterdosierung von lebensrettenden Medikamenten führen kann; 
- anatomische Besonderheiten, z. B. die Anatomie von Thorax und Kehlkopf beim Säugling;

- erschwerte Kommunikationsmöglichkeit und Anamneseerhebung bei kleinen Kindern;

- logistische Probleme, z. B. die ungenügende Fixation auf den standardisierten Rettungstragen sowie eine unvollständige Ausstattung für Kindernotfälle;

- die ggf. erhebliche emotionale Belastung für Notarzt und Rettungsdienstpersonal

(Gortner 1998, Helm et al. 2005, Jöhr 2005, Schürmann und Lemburg 2001).

Daraus ergibt sich die besondere Bedeutung einer fundierten Vorbereitung auf pädiatrische Notfälle.

\subsection{Anteil präklinischer Kindernotfälle}

Kinder unter 15 Jahren haben in Deutschland einen Anteil von 13,9 \% an der Gesamtbevölkerung (Statistisches Bundesamt [DESTATIS] 2006). Ihr Anteil an allen präklinischen Notarzteinsätzen beträgt - je nach Untersuchung - 5 bis $10 \%$ (siehe Tabelle 12). Somit ist die klinische Erfahrung der meisten Notfallteams im Umgang mit pädiatrischen Notfällen eher gering. Während Notfälle im Säuglings- und Kleinkindesalter bei den Untersuchungen von Applebaum mit $68 \%$ und Stopfkuchen et al. mit $62 \%$ überwiegen, findet sich in anderen Studien ein Überwiegen von Klein- und Schulkindern (Applebaum 1985, Tsai und Kallsen 1987, Suominen et al.1996, Stopfkuchen et al. 1999, Albrech et al. 2000, Nagele und Kroesen 2000, Richard et al. 2006, Eich et al. 2009a). Der Anteil präklinischer Kindernotfälle am Gesamteinsatzaufkommen im Untersuchungszeitraum betrug in Göttingen 6,3\%, wobei der Anteil im luftgebunden Rettungsdienst bei 8,5 \% lag. Damit liegen diese Ergebnisse im Bereich anderer Erhebungen (Tabelle 12). 


\begin{tabular}{|c|c|c|}
\hline Untersuchung & $\begin{array}{l}\text { Anteil pädiatrischer } \\
\text { Notfälle }\end{array}$ & Land, Erscheinungsjahr \\
\hline Eich et al. a) & $\begin{array}{l}\text { 6,3 \% } \\
\text { Luft: 8,5 \% } \\
\text { Boden:5,2\% }\end{array}$ & Deutschland, 2009 \\
\hline Richard et al. & $5-10 \%$ & Kanada, 2006 \\
\hline Gries et al. & $\begin{array}{l}\text { Luft: 5,9\% } \\
\text { Boden: 4,9\% }\end{array}$ & Deutschland, 2005 \\
\hline Schlechtriemen et al. a) & $\begin{array}{l}\text { Luft: 9,2 \% } \\
\text { Boden: 4,2 \% }\end{array}$ & Deutschland, 2005 \\
\hline Möller et al. & $3,5 \%$ & Deutschland, 2002 \\
\hline Bartels & $5 \%$ & Deutschland, 2001 \\
\hline Nagele und Kroesen & $5,1 \%$ & Österreich, 2000 \\
\hline Albrech et al. & са. $11 \%$ & Deutschland, 2000 \\
\hline Murdock et al. & $6 \%$ & USA, 1999 \\
\hline Brambrink & $5-10 \%$ & Deutschland, 1998 \\
\hline Kumar et al. & са. $10 \%$ & USA, 1997 \\
\hline Suominen et al. & $8 \%$ & Finnland, 1996 \\
\hline Svenson et al. & $10 \%$ & USA, 1996 \\
\hline Hirschfeld & $10 \%$ & USA, 1995 \\
\hline Graham et al. & $4 \%$ & USA, 1993 \\
\hline Seidel & $10 \%$ & USA, 1986 \\
\hline Applebaum & $5 \%$ & Israel, 1985 \\
\hline
\end{tabular}

Tabelle 12 Anteil präklinischer Kindernotfälle in der Literatur

Die meisten der von uns befragten Notärzte (55,8 \%), überwiegend im Alter zwischen 31 bis 40 Jahren und mehrheitlich mit einer Berufserfahrung als Notarzt zwischen 2 bis 5 Jahren, schätzten die prozentuale Häufigkeit pädiatrischer Notfälle im ärztlichen Rettungsdienst mit einer Häufigkeit zwischen 3,1 bis $6 \%$ ein. Diese Einschätzung liegt nahe an den realen Einsatzdaten des Göttinger Notarztdienstes (6,3\%). Dabei gingen die Befragten von einem Überwiegen der Altersgruppen Kleinkinder und Schulkinder aus. Je 41,8 \% erwarteten einen Anteil klein- und schulkindlicher Notfälle zwischen 31-50 \%, während Notfälle im Säuglingsalter laut Einschätzung von 46,5 \% der Befragten mit einer Häufigkeit von weniger als $10 \%$ auftreten. 


\subsection{Einteilung der Kindernotfälle}

\subsubsection{Abgrenzung pädiatrischer Patienten von erwachsenen Patienten}

Eine Differenzierung zwischen Kindern und Erwachsenen ist in der Notfallmedizin aufgrund der abweichenden Anatomie und der daraus resultierenden Unterschiede in Diagnostik und Therapie sinnvoll. Darüber hinaus erlaubt eine Differenzierung von Kindernotfällen nach Altersgruppen, Unterschiede in der Häufigkeit des Auftretens von Diagnosen und Interventionen herauszuarbeiten. Die wesentlichen anatomischen und physiologischen Unterschiede zwischen pädiatrischen und erwachsenen Patienten, die in der Notfallmedizin unbedingt Beachtung finden müssen, lassen sich wie folgt zusammenfassen:

- Das bei Kindern im Vergleich zu Erwachsenen erhöhte Totraumvolumen (40\% des Atemzugvolumens bei Kindern gegenüber 30 \% bei Erwachsenen).

- Der Kehlkopf des Neugeborenen und des jungen Säuglings liegt auf Höhe des 3. oder 4. Halswirbelkörpers und damit rund zwei Wirbelkörper höher als bei Erwachsenen. Ferner liegt er weiter ventral als bei größeren Kindern und bei Erwachsenen.

- Die Zunge des Säuglings ist relativ größer als die des Erwachsenen.

- Mit steigendem Lebensalter sinkt der relative Flüssigkeitsbedarf, da die Evaporationsrate sich vermindert und die Konzentrationsfähigkeit der Niere erhöht.

- Die Ruheatemfrequenz sinkt bei Kindern vom Neugeborenenalter (35 bis 50 Atemzüge pro Minute) bis zum Schulkindalter (12 bis 15 Atemzüge/Minute)

(Gortner 1998, Jöhr 2005, Nicolai 2001).

In der vorliegenden Untersuchung wurde eine obere Altersbegrenzung der Kinder auf $\leq 14$ Lebensjahre gesetzt. Damit werden den vom Erwachsenenalter abweichenden Besonderheiten dieser Notfälle Rechnung getragen. Diese Einteilung erfolgte in Übereinstimmung mit zahlreichen Voruntersuchern (Albrech et al. 2000, Applebaum 1985, Moront et al. 1996, Nagele und Kroesen 2000, Schlechtriemen et al. 2005a). Allerdings gibt es keine national oder international verbindlich festgelegte obere Grenze des Kindesalters.

\subsubsection{Abgrenzung der Entwicklungsperioden des Kindes}

Innerhalb der Patientengruppe „Kinder“ ergibt sich aus dem Wachstum und dem Erlernen von Fähigkeiten und Fertigkeiten eine große Variabilität. So ist die Fähigkeit zur Kommunikation bei Kindern noch unzureichend ausgebildet, woraus sich ergibt, dass sich der behandelnde 
Arzt nicht auf die Angaben des kleinen Patienten beispielsweise bezüglich Schmerzdauer, Schmerzlokalisation und Schmerzqualität verlassen kann (Albrech et al. 2000).

Die Besonderheiten der kindlichen Anatomie und Physiologie oder auch des kindlichen Verhaltens prädestinieren darüber hinaus zu typischen Notfallsituationen in den verschiedenen Altersstufen, wie beispielsweise dem plötzlichen Kindstod bei Säuglingen oder dem Unfalltrauma bei Schulkindern (Brambrink 1998). Eine Einteilung in Altersgruppen bzw. Entwicklungsperioden erscheint daher sinnvoll (siehe Tabellen 13 und 14).

\begin{tabular}{l|l|l|l|l} 
& Neugeborenes & Säugling & Kleinkind & Schulkind \\
\hline Alter & $\leq 4$ Wochen & $\begin{array}{l}<1 \text { Lebensjahr } \\
\text { (bis zum 1. Geburtstag) }\end{array}$ & $1 .-6$. Lebensjahr & $>$ 6 Lebensjahre
\end{tabular}

Tabelle 13 Altersgruppen bzw. Entwicklungsperioden des Kindes nach Koletzko 2004

\begin{tabular}{l|l|l|l|l|l} 
& $\begin{array}{l}\text { Motorische } \\
\text { Fähigkeiten }\end{array}$ & Sprache & $\begin{array}{l}\text { Angaben zur } \\
\text { Schmerzlokalisation }\end{array}$ & $\begin{array}{l}\text { Kognitive } \\
\text { Fähigkeiten }\end{array}$ & Autonomie \\
\hline $\begin{array}{l}\text { Neugeborenes } \\
\text { (< } 4 \text { Wochen) }\end{array}$ & Nein & Nein & Nein & Nein & Nein \\
$\begin{array}{l}\text { Säugling } \\
(4 \text { Wochen bis }\end{array}$ & Eingeschränkt & Nein & Nein & Nein \\
$\begin{array}{l}1 \text { LJ) } \\
\text { Kleinkind } \\
(1-5 \text { LJ) } \\
\begin{array}{l}\text { Schulkind } \\
(6-14 \text { LJ) }\end{array}\end{array}$ & Ja & Eingeschränkt & Nein & Eingeschränkt & Nein \\
\end{tabular}

Tabelle $14 \quad$ Unterschiede der Entwicklungsperioden des Kindes (Flake und Heinichen 2010, S. 6)

Eine vereinfachte Form dieser Alterseinteilung wurde in den aktuellen Leitlinien des International Liaison Committee on Resuscitation (ILCOR) und des European Resuscitation Council (ERC) berücksichtigt, da dadurch die Behandlung des kindlichen Notfallpatienten beispielsweise hinsichtlich des Kompression-Ventilations-Verhältnisses bei der Reanimation auf sein Alter abgestimmt werden kann (Francis et al. 2008).

\subsection{Häufigkeit pädiatrischer Notfälle}

Notfälle im Schul- und Kleinkindalter traten im Zeitraum der vorliegenden Untersuchung mit 43,8 \% beziehungsweise 42,1 \% am häufigsten auf, mit einem deutlichen Abstand zur Inzidenz von Säuglingsnotfällen (14,0 \%). Der Literatur zufolge überwiegt in der Häufigkeit der Notfalleinsätze die Altersgruppe der Kleinkinder (Applebaum 1985, Babl et al. 2001, Eckstein et al. 2002, Fifield et al. 1984, Murdock et al. 1999, Nagele und Kroesen 2000, Richard 
et al. 2006, Stopfkuchen et al. 1999, Suominen et al. 1996). Tsai und Kallsen (USA 1987), Albrech et al. (Deutschland 2000) sowie Nagele und Kroesen (Österreich 2000) fanden in ihren Untersuchungen pädiatrischer Notfälle eine bimodale Altersverteilung mit einem ersten Peak im ersten Lebensjahr und einem Ansteigen von Notfällen während der Adoleszenz (Albrech et al. 2000, Nagele und Kroesen 2000, Tsai und Kallsen 1987). In der Untersuchung präklinischer Kindernotfälle im Saarland findet sich in Übereinstimmung mit unseren Ergebnissen ein knappes Überwiegen von Notfällen im Schulkindalter, dicht gefolgt von der Anzahl präklinischer Kindernotfälle im Kleinkind- und Säuglingsalter (Schlechtriemen et al. 2005a). Auch Richard et al. bestätigen 2006 die in der vorliegenden Untersuchung ermittelte Altersverteilung (Richard et al. 2006). In einer allgemeinen Patientenanalyse des Notarztdienstes der Universität Heidelberg zeigen Bernhard et al., dass der Altersdurchschnitt der versorgten Notfallpatienten im Laufe der Jahre signifikant anstieg, wobei der Anteil von Kindernotfällen im Jahre 2004 2,9\% betrug und damit geringfügig niedriger war als bei Voruntersuchungen in den Jahren 1984 und 1992, wo er 4,0 \% betrug. Betrachtet man jedoch die absolute Anzahl der Notfalleinsätze, so hat sich die Gesamtzahl pädiatrischer Patienten von 36 im Jahre 1984 auf 109 im Jahre 2004 verdreifacht (Bernhard et al. 2006).

\subsection{Schweregrad pädiatrischer Notfälle}

Die Einteilung des Schweregrades der pädiatrischen Notfälle erfolgte mithilfe des weit verbreiteten NACA- (National Advisory Committee on Aeronautics) Score. Der NACA-Score ist ein einfaches Ordnungssystem zur Bestimmung des Schweregrades einer Erkrankung oder einer Verletzung. Er ist messwertunabhängig und lässt sich sowohl auf Erkrankungen als auch Verletzungen anwenden. Mithilfe des NACA-Scores wird auch definiert, wann die unbedingte Indikation zur Alarmierung eines Notarztes besteht. In der Literatur wird diese ab einem Schweregrad NACA $\geq$ III (schwere, jedoch nicht lebensgefährliche Erkrankung oder Verletzung) gesehen (Nagele und Kroesen 2000, Weiss et al. 2001).

Ein relevanter Kritikpunkt am NACA-Score ist seine Abhängigkeit von der weitgehend subjektiven Beurteilung durch das Notfallteam und somit auch von dessen Ausbildungsstand und persönlicher Erfahrung (Schlechtriemen et al. 2005b, Weiss et al. 2001). So zeigen Knapp et al. auf, dass Notärzte mit weniger Einsatzerfahrung tendenziell eine geringere Erkrankungsschwere annehmen als Notärzte mit größerer Einsatzerfahrung (Knapp et al. 2008).

Umgekehrt scheinen Laien kindliche Notfälle eher schwerwiegender einzuschätzen und Akutsituationen beim Kind grundsätzlich als lebensbedrohliche Notfälle zu empfinden. 
Dies führt dazu, dass die Mehrzahl der pädiatrischen Notfälle retrospektiv mit niedrigeren Schweregraden (NACA I bis III) bewertet werden. Darüber hinaus gibt es jedoch regelmäßig vitalbedrohliche und zeitkritische Kindernotfälle, wie Atem- und Kreislaufstillstand oder schwere Traumata, die ein rasches, konsequentes und sicheres Handeln erfordern (Stenke 2004, Suominen et al. 1996).

In Göttingen wurden 72,7 \% der erfassten Kindernotfälle durch den Notarzt vor Ort als weniger schwerwiegend (NACA-Score I-III) bewertet. Dies gilt vor allem für die Altersgruppe der Schulkinder. Notfälle bei Säuglingen und Kleinkindern wurden tendenziell schwerwiegender eingeschätzt. Ähnliche Gruppenunterschiede ergaben Untersuchungen des Münchner Kindernotarztdienstes und des Lübecker Notarztdienstes (Ballnus et al. 2002, Stenke 2004). Ballnus et al. führen dies auf die Einschätzung der Situation durch die Eltern zurück, weshalb ihrer Ansicht nach ein Schwerpunkt der notärztlichen Ausbildung auf die psychische Intervention und menschliche Betreuung der kleinen Patienten und deren Angehörigen gelegt werden sollte (Ballnus et al. 2002). Durchschnittlich werden Notfälle bei Kindern in weniger als einem Drittel der Fälle mit höheren NACA-Scores (IV-VII) bewertet, wobei pädiatrische Notfälle im Vergleich zu Erwachsenen generell niedrigere Schweregrade aufweisen (Eich et al. 2009a, Stenke 2004).

Fifield et al. fanden bereits 1984, dass fast $80 \%$ der untersuchten kindlichen Notfälle im Rettungsdienst von geringerem Schweregrad waren (Fifield et al. 1984). Die Autoren führen dies auf unangebrachte Nutzung der Rettungsdienste durch die Bevölkerung oder auf eine Überreaktion des „Systems“ zurück. Dabei ist der kindliche Notfall jedoch in seiner tatsächlichen Schwere vom Laien und auch vom Leitstellendisponenten nur unzureichend einzuschätzen, sodass sich in der Regel ein Notarzt vor Ort selbst ein Bild machen muss. Oft wird jedoch auch der Notarzt vor Ort mit seiner begrenzten Ausrüstung nur eine sehr ungenaue Einschätzung über den tatsächlichen Schweregrad geben können.

In einer Untersuchung des allgemeinen Notarztaufkommens bringen Gries et al. in ihrer Einschätzung über die Zukunft der präklinischen Notfallmedizin in Deutschland die Frage auf, ob sich die zunehmende Beanspruchung des Notarztdienstes tatsächlich durch eine steigende Anzahl schwer erkrankter oder verletzter Patienten erklären lässt oder ob vielmehr ein relevanter Teil der Einsätze nicht indiziert ist (Gries et al. 2003).

Laut Schlechtriemen et al. finden sich schwerwiegende pädiatrische Notfälle vor allem in der Luftrettung sowie in der Altersgruppe der Säuglinge (Schlechtriemen et al. 2005a). Dies bestätigen Untersuchungen des Luftrettungsdienstes im Saarland durch Albrech et al. sowie die Evaluation pädiatrischer Traumapatienten im Luftrettungszentrum Ulm durch Helm 
et al. (Albrech et al. 2000, Helm et al. 1999). Moront et al. hingegen beschreiben im Luftrettungsdienst ein relatives Überwiegen von Kindern mit geringfügigen oder mittelschweren Verletzungen (Moront et al. 1996).

Nagele und Kroesen schließen in ihrer Untersuchung des Notarztdienstes in Österreich eine Notarztindikation in 44,6 \% aller Notfälle mit einem NACA-Score $\geq 3$ in der retrospektiven Datenauswertung aus (Nagele und Kroesen 2000).

\subsection{Sicherheit im Umgang mit den Altersgruppen}

Ihr eigenes Sicherheitsgefühl schätzte das befragte Notarztkollektiv vor allem im Umgang mit Neugeborenen als eher gering ein. Das Sicherheitsempfinden der Notärzte bei präklinischen Kindernotfällen korreliert eng mit dem Lebensalter des Kindes (je älter, desto sicherer) und ihrer eigenen mutmaßlichen Expertise bezüglich der betreffenden Diagnose bzw. Intervention.

In einer Befragung von Notärzten durch Bartels wurde als schwierigste Altersgruppe von 73 \% der Befragten die Altersgruppe der 0- bis 2-Jährigen angegeben (Bartels 2001).

In unserer Befragung wurde als Altersgruppe, in der die geringste Routine in der medizinischen Versorgung empfunden wurde, die der Neugeborenen und Säuglinge genannt. Nach Eich et al. hat die Mehrzahl der Anästhesisten in Deutschland nur eine sehr begrenzte kinderanästhesiologische Erfahrung (Eich et al. 2006). In einer 1994 durch Zink et al. durchgeführten Umfrage waren 50 \% der aktiven Notärzte nicht in der Lage, ein schwer verletztes Kind präklinisch adäquat zu versorgen. Weiterhin zeigte diese Untersuchung, dass sich 88 \% der befragten Notärzte am meisten vor kindlichen Notfällen fürchteten (Zink et al. 2004).

\subsection{Transport und Transportziel}

87,4 \% der erfassten pädiatrischen Notfälle wurden durch Boden- oder Lufttransport in eine medizinische Versorgungseinrichtung verbracht. Der Transport erfolgte in 43,5 \% luft- (RTH) und in 56,5 \% bodengebunden (RTW). Vor allem Schulkinder, mit ihrer relativ hohen Prävalenz an Traumata, wurden mit 46,6 \% häufig luftgebunden transportiert, während dieser Anteil bei Kleinkindern und Säuglingen nur 42,3 \% bzw. 37,3 \% betrug.

Transportziel war in 80,3 \% der Fälle die Universitätsmedizin Göttingen, welche als tertiäres pädiatrisches Zentrum die Region Göttingen versorgt. In 7,1 \% der Fälle erfolgte der 
Transport in ein Verbrennungs- bzw. Replantationszentrum oder in Einzelfällen in ein peripheres Krankenhaus. Bei 12,6 \% der pädiatrischen Notfälle erfolgte kein Transport.

In der Untersuchung von Tsai und Kallsen fand ein Transport in 83,6 \% der Fälle statt, wobei in 78,8 \% ein Krankenhaus der Grund-/Regelversorgung angefahren wurde (Tsai und Kallsen 1987). Gemäß Nagele und Kroesen (Österreich) erfolgte ein Transport in 93 \% der Fälle (Nagele und Kroesen 2000). Bei Applebaum erfolgte ein Transport bei 85,3 \% der pädiatrischen Notfälle, bei Gerlacher et al. in 80,2 \% (Applebaum 1985, Gerlacher et al. 2001). Damit besteht eine weitgehende Übereinstimmung mit unseren Untersuchungsergebnissen. Im Gegensatz zu diesen Ergebnissen erfolgte bei Erhebungen von Richard et al. mit 27,9 \% bei einem relativ großen Teil der pädiatrischen Notfälle kein Transport und bei Stopfkuchen wurden sogar 31 \% der pädiatrischen Notfälle ambulant behandelt (Richard et al. 2006, Stopfkuchen et al. 1999).

Oft ist die Art des Transportmodus vom Schweregrad der Verletzung oder Erkrankung des Kindes abhängig. Vor allem schwere Notfälle wurden häufiger durch die Luft- als durch die Bodenrettung versorgt (Schlechtriemen et al. 2005a). Dieses Ergebnis begründen die Autoren damit, dass bestimmte Verletzungsmuster (SHT, Verbrennungen und Verbrühungen) von der Behandlung in überregionalen Zentren profitieren, welche mit Luftrettungsmitteln schneller zu erreichen sind. Auch die vorliegende Studie zeigt dass, schwerwiegende Notfälle, wie Ertrinken, SIDS, Trauma und Schädel-Hirn-Trauma, im überwiegenden Teil durch die Luftrettung versorgt werden.

\subsection{Diagnosen}

\subsubsection{Häufigkeit pädiatrischer Notfalldiagnosen}

Die häufigsten präklinisch-pädiatrischen Notfallgruppen im Göttinger Notarztdienst waren Traumata, respiratorische Störungen und Krampfanfälle. Auf dem RTH stationierte Notärzte behandelten dabei einen besonders hohen Anteil von Traumata (61,1 \%). Dazu zählten Kopfverletzungen, Verbrennungen und Verbrühungen sowie Frakturen. Entsprechend dieser Häufigkeitsverteilung sollten Notärzte ausreichend mit diesen Notfällen vertraut sein, denn ähnliche Prävalenzen finden sich auch in Untersuchungen von Albrech et al. 2000, Babl et al. 2001, Möller et al. 2002, Murdock et al. 1999, Richard et al. 2006, Schlechtriemen et al. 2005a sowie Suominen et al. 1996.

Der Vergleich zwischen Luft- und Bodenrettung durch Schlechtriemen et al. zeigt einen deutlich höheren Anteil polytraumatisierter Kinder und vitalbedrohlicher pädiatrischer 
Notfälle in der Luft- im Vergleich zur Bodenrettung (Schlechtriemen et al. 2005a). In der Untersuchung durch Schlechtriemen dominieren in der Luftrettung bei Kleinkindern SchädelHirn-Traumata (SHT) und bei Schulkindern Extremitätenverletzungen, welche die Anzahl der Schädel-Hirn-Traumata in dieser Altersgruppe übersteigen (Schlechtriemen et al. 2005a).

\subsubsection{Häufigkeiten pädiatrischer Notfalldiagnosen in den Altersgruppen}

In der Altersgruppe der Säuglinge dominierten als Notfalldiagnose respiratorische Störungen mit einem Anteil von 34,5 \%, insbesondere Aspiration und plötzlicher Kindstod (SIDS). Laut Möller et al. sind 80 \% aller Todesfälle bei Kindern unter 14 Lebensjahren primär respiratorisch bedingt (Möller et al. 2005). Der plötzliche Kindstod ist eine auf die Altersgruppe der Säuglinge begrenzte Diagnose. Er wird definiert als plötzlicher Tod eines Säuglings ( $<1$ Lebensjahr) mit wahrscheinlichem Beginn der zum Versterben führenden Episode im Schlaf. Dabei ist, trotz einer gründlichen postmortalen Untersuchung, einschließlich einer vollständigen Autopsie und Beurteilung der Krankengeschichte und Todesumstände, keine adäquate Todesursache zu finden (Poets 2005).

Eher selten fanden sich bei Säuglingen Fälle mit der Diagnose ,Asthma‘ (0,6 \%). Die Diagnose ,Epiglottitis‘ trat in dieser Altersgruppe nicht auf. Die Diagnose ,Asthma‘ findet sich überwiegend in den Altersgruppen Schul- und Kleinkinder, wobei Schulkinder mehr als doppelt so häufig betroffen sind. Die Diagnose ,Epiglottitis‘ wurde während des neunjährigen Untersuchungszeitraums bei nur einem Kleinkind dokumentiert.

In der Häufigkeit der Diagnosen folgten in der Altersgruppe ,Säuglinge“ der Krampfanfall (17,2 \%), vorwiegend fieberassoziiert, und das Trauma (16,0\%), mit einem Gesamtanteil der Schädel-Hirn-Traumata (SHT) von 3,4 \%. Schlechtriemen et al. hingegen beschreiben das SHT als führende Verletzungslokalisation und konnten dieses in zwei Drittel aller Traumata in der Altersgruppe der Säuglinge nachweisen (Schlechtriemen et al. 2005a).

In der Altersgruppe der Kleinkinder fanden sich am häufigsten Krampfanfälle (43,2 \%), ebenfalls vorwiegend fieberassoziiert. Altemeyer et al. beschreiben den fieberassoziierten Krampfanfall als typischen Notfall des Kleinkindes-, aber auch des Säuglingsalters (Altemeyer et al. 2000). Laut Keimer beträgt die Inzidenz zerebraler Krampfanfälle bei Kindern 4-5 \%, wobei manifeste Epilepsien in der Gesamtbevölkerung mit einer Häufigkeit von 0,5-1 \% auftreten (Keimer 1999). Gemäß einer Untersuchung von Pallin et al. sind Krampfanfälle für $1 \%$ aller kindlichen Notfälle in Notaufnahmen verantwortlich, wobei fieberassoziierte Krampfanfälle eine besonders wichtige Untergruppe darstellen (Pallin et al. 2008). Nach 
Richard et al. sind Krampfanfälle die häufigsten nicht-verletzungsbedingten Ursachen für einen Transport in ein Krankenhaus in der Altersgruppe der Kleinkinder (Richard et al. 2006).

Traumata traten in dieser Altersgruppe prozentual häufiger auf (19,5\%) als in der Altersgruppe der Säuglinge, während respiratorische Störungen prozentual seltener auftreten (21,8 \%). Auch der Anteil der Kopfverletzungen (SHT) und Verbrennungen war häufiger im Vergleich zur Altersgruppe der Säuglinge. Kindliche Verbrennungen oder Verbrühungen wurden gemäß unserer Untersuchung mit einer Gesamthäufigkeit von 2,5 \% aller pädiatrischen Notfälle behandelt. Laut Jester et al. stellt gerade die relativ geringe Inzidenz von Verbrennungen und Verbrühungen im Kindesalter eine besondere Herausforderung für den Notarzt dar (Jester et al. 2006). Dabei sind 90 \% dieser Verletzungen nach Jester et al. durch Verbrühungen bedingt und es besteht ein Häufigkeitsgipfel zwischen ein bis drei Jahren.

Reichmann et al. beschreiben den Unfalltod als häufigste Todesursache bei Kindern, die, so meinen die Autoren, in der Bevölkerung häufig unterschätzt wird (Reichmann et al. 1998). Die größte Verletzungshäufigkeit haben dabei Extremitätenverletzungen, während die zweithäufigste Verletzung bei den 3- bis 16-jährigen das Schädel-Hirn-Trauma darstellte (Reichmann et al. 1998).

Bei Schulkindern fand sich in der vorliegenden Untersuchung die Diagnose Trauma als häufigste dokumentierte Diagnose mit einem prozentualen Anteil von 44,4 \%. Dieser hohe Anteil lässt sich mit der steigenden Aktivität des älter werdenden Kindes außerhalb des häuslichen Umfeldes erklären.

Insgesamt steigt gemäß unseren Daten die Inzidenz von Traumanotfällen mit zunehmendem Lebensalter der Kinder, was Untersuchungen von Helm et al. 1999, Svenson et al. 1996 und Søreide et al. 2009 bestätigen. Dafür ist im Wesentlichen die Zunahme der Verkehrsunfälle verantwortlich (Helm et al. 1999, Svenson et al. 1996, Søreide et al. 2009).

Bei Schlechtriemen et al. nimmt vor allem der Anteil der Extremitätenverletzungen in dieser Altersgruppe zu (Schlechtriemen et al. 2005a). Auch in der vorliegenden Untersuchung überwogen anderweitige Traumata gegenüber dem SHT und den Verbrennungen und Verbrühungen, die bei Schulkindern seltener sind als bei Kleinkindern. Krampfanfälle (16,0 \%) und respiratorische Störungen (15\%) zeigten bei Schulkindern etwa die gleiche Inzidenz, wobei hier der Anteil des Asthma bronchiale und der nicht-fieberassoziierten Krampfanfälle anstieg. 


\section{9 Therapeutische Interventionen}

\subsubsection{Intubation und kardiopulmonale Reanimation}

Mit Ausnahme der Anlage eines periphervenösen Zugangs sind weitergehende therapeutische Interventionen beim kindlichen Notfall insgesamt eher selten. Dabei überwogen in unserer Studie mit 96 Fällen (4,2 \%) die tracheale Intubation und mit 52 Fällen (2,3 \%) die kardiopulmonale Reanimation. Intubiert wurden vor allem Schulkinder, während die Reanimation am häufigsten bei Säuglingen durchgeführt wurde. Setzt man die Anwendungen allerdings mit den jeweiligen Fallzahlen in den Altersgruppen in Beziehung, so ist auch der prozentuale Anteil der intubierten Säuglinge an der Gesamtzahl der behandelten Säuglinge höher (7,5 \%) als der Anteil der intubierten Klein- und Schulkinder (3,2 \% und 4,1\%). Dieses Ergebnis lässt sich mit dem Diagnosenspektrum der pädiatrischen Altersklassen erklären, so tritt im Säuglingsalter die Diagnose des plötzlichen Kindstodes (SIDS) als Intubationsindikation in den Vordergrund.

Die präklinische Intubation von Kindern ist nicht unumstritten. So ist die notfallmäßige Intubation beim kindlichen Patienten durch die Anatomie, infolge eines unzulänglichen Trainings der Sanitäter und Notärzte und dem seltenen Anwendungsbedarf erschwert (Brownstein et al. 1996, DiRusso et al. 2005, Jöhr 2005). Gemäß Gausche et al. und Roberts et al. verbessert eine präklinische endotracheale Intubation die Überlebenschancen des pädiatrischen Patienten in einem schnell transportierenden Rettungsdienstsystem nicht (Gausche et al. 2000, Roberts et al. 2005). Gausche et al. zeigen in ihrer Untersuchung mit Paramedics weiterhin auf, dass die Intubation bei intubationspflichtigen pädiatrischen Patienten jedoch nur bei 57-62 \% der Kinder erfolgreich war, wodurch die Aussagekraft dieser Studie abgeschwächt erscheint. Boswell et al. beschreiben eine deutlich höhere Zahl erfolgloser Intubationsversuche bei Kindern im Vergleich zu erwachsenen Notfallpatienten (Boswell et al. 1995). Jewkes findet eine 33 \%ige Komplikationsrate bei der Anwendung der trachealen Intubation bei Kindern (Jewkes 2001). Angesichts eines hohes Fehlerpotentials der präklinischen Intubation halten Wang et al. und Roberts et al. profunde Kenntnisse des pädiatrischen Atemwegsmanagements für entbehrlich (Wang et al. 2006, Roberts et al. 2005). In der Literatur findet sich sowohl die Aufforderung zur möglichst frühzeitigen Intubation bei Kindern (Brambrink 2002) als auch die gegensätzliche Empfehlung (Brownstein et al. 1996, Nicolai 2001).

In einer Untersuchung von Eich et al. werden insgesamt 82 präklinisch intubierte Kinder (4,0 \% des gesamten pädiatrischen Patientenanteils) untersucht. Dabei werden 58 Kinder 
durch Notärzte mit anästhesiologischer Weiterbildung mit einer Erfolgsrate von 98,3 \% endotracheal intubiert. Weitere 24 pädiatrische Notfallpatienten werden durch Notärzte anderer Spezialisierung versorgt. Hier wurde keine Erfolgsrate bestimmt. 86,6 \% der 82 pädiatrischen Patienten überlebten bis zur Ankunft im Krankenhaus und 76,8 \% bis zur Entlassung aus dem Krankenhaus (Eich et al. 2009b).

Blanco-Ons Fernández et al. finden im Gegensatz zu unserer Untersuchung in ihrer Studie in Galizien (Spanien) eine hohe präklinische Intubationsrate von 84 \%, ohne Angabe einer Erfolgsrate, was durch die Autoren auf einen hohen Erfahrungslevel der Sanitäter und Notfallmediziner im Umgang mit präklinischen pädiatrischen Notfällen zurückgeführt wird (Blanco-Ons Fernández et al. 2007). Richard et al. berichten hingegen über eine seltene Anwendung der Atemwegssicherung (Richard et al. 2006).

Die Intubation wird trotz der Gefahren und Schwierigkeiten als wichtige Intervention bei der Wiederbelebung pädiatrischer Notfallpatienten angesehen (Easley et al. 2000, Graham 2004). Nach Ansicht der Autoren sollten zukünftige Leitlinien die adäquate Expertise des Anwenders besser berücksichtigen.

Der Literatur zufolge werden übereinstimmend mit unseren Ergebnissen vor allem Kinder unter sechs Lebensjahren reanimiert (Aijin et al. 1989, Nakayama et al. 1989, Sirbaugh et al. 1999).

Hingegen wurde bei nur sechs Kindern in Göttingen (0,3 \%) im Rahmen der Reanimation eine Defibrillation durchgeführt. Drei der defibrillierten Kinder befanden sich im Säuglingsalter und drei im Kleinkindalter. Somit scheint die Defibrillation bei Kindern eine besonders seltene Intervention zu sein. Dies deckt sich mit der insgesamt niedrigen Prävalenz von Kammerflimmern und pulsloser Kammertachykardie bei Kindern (ILCOR 2005a). Die kardiopulmonale Reanimation (CPR) beim Kind ist mit besonderen Anforderungen an die Einsätzkräfte im Rettungsdienst verbunden. Da der vollständige Atem-Kreislauf-Stillstand bei Kindern ein eher seltenes Ereignis ist, haben Notfallteams im Allgemeinen wenig Übung mit der kardiopulmonalen Reanimation von Kindern (Atkins et al. 2009, Chen und Hsiao 2008, Donoghue et al. 2006, Blanco-Ons Fernández et al. 2007, Kuisma et al. 1995, Sirbaugh et al. 1999).

Wegen des geringeren Anwendungsbedarfs der Reanimation beim Kind sind Untersuchungen über den Atem-Kreislauf-Stillstand bei Kindern und ihre Reanimation entsprechend seltener als bei erwachsenen Patienten (Kuisma et al. 1995). Ursachen, die zum kindlichen Kreislaufstillstand führen, sind vor allem respiratorische Ursachen (Hypoxämie) gefolgt von Hypovolämie vor allem im Rahmen von Trauma, Sepsis oder Dehydratation sowie neurologi- 
sche Störungen. Nach Kuisma et al. führen vor allem SIDS, Trauma und seltener, im Gegensatz zum Erwachsenen, kardiale Ursachen zum kindlichen Atem-Kreislauf-Stillstand (Kuisma et al. 1995, Ong et al. 2006).

\subsubsection{Thoraxdrainage und intraossäre Punktion}

Viermal wurden im Untersuchungszeitraum Thoraxdrainagen in den Altersgruppen der Kleinkinder und Schulkinder gelegt. Die intraossäre (IO) Punktion kam bei 15 Kindern als Gefäßzugang zur Anwendung, drei Fünftel davon in der Altersgruppe der Säuglinge, zwei Fünftel im Kleinkindalter. Die intraossäre Punktion hat in den internationalen Leitlinien einen hohen Stellenwert, obwohl ihre Anwendung durch die persönlichen Fertigkeiten und technischen Möglichkeiten häufig nach wie vor limitiert ist (Biarent et al. 2005, Smith et al. 2005).

Die Indikation zur intraossären Punktion besteht dann, wenn ein Gefäßzugang dringlich erforderlich ist (z. B. Atem-Kreislaufstillstand, Kreislaufschock) und die Anlage eines periphervenösen Gefäßzugangs nach maximal drei Versuchen misslingt (Bernhard et al. 2008, Biarent et al. 2005).

Weiss et al. beschreiben eine primäre und eine sekundäre Indikation zur intraossären Infusionstechnik (Weiss et al. 2009). Die primäre Indikation (sofortiger IO-Zugang) besteht nach Ansicht der Autoren beim Kind jünger als sechs Jahre mit Atem-Kreislauf-Stillstand, während die sekundäre Indikation beim vital-gefährdeten Säugling oder Kind gestellt wird, bei dem der Versuch eines i. v. Zugangs nach dreimaligem Versuch oder nach Ablauf von 90120 Sekunden erfolglos verlief. Helm et al. beschreiben die intraossäre Punktion als schnelle und sichere Notfallalternative bei verzögertem, schwierigem oder gar unmöglichem konventionellen Gefäßzugang beim Kind (Helm et al. 2005). Laut Gervais kann der intraossäre Zugangsweg problemlos auch bei Kindern älter als sechs Lebensjahren angewendet werden (Gervais 2001).

Nach Helm et al. hat die intraossäre Punktion insbesondere bis zum sechsten Lebensjahr praktische Bedeutung (Helm et al. 2005). Eine sehr seltene Komplikation der intraossären Punktion ist die Osteomyelitis. Häufiger treten Frakturen, Gewebenekrosen, Kanülendislokation und Punktionen der Wachstumsfuge auf (Helm et al. 2005). Jewkes fand eine fünfzehnprozentige Komplikationsrate bei der Anwendung der intraossären Punktion (Jewkes 2001).

Die intraossäre Punktion wird trotz einfacher, effektiver Handhabung - und obwohl sie seit 1994 in den pädiatrischen Leitlinien des ERC ausdrücklich empfohlen wird - nur relativ selten in der präklinischen Medizin angewendet (Zink et al. 2004). Obwohl alle in der vorliegen- 
den Untersuchung befragten Notärzte mit den aktuellen Leitlinien vertraut und in der Technik trainiert sind, ist die Inzidenz der IO-Punktion in Göttingen im Untersuchungszeitraum ähnlich gering wie in anderen Zentren (Helm et al. 2007, Smith et al. 2005).

Tendenziell erfolgen invasive Interventionen häufiger in der Luft- als in der Bodenrettung. In der Literatur findet sich vor allem die Anlage intravenöser Zugänge als häufigste Intervention (Babl et al. 2001, Sirbaugh et al. 1999). Gausche et al. wiesen nach, dass einfache Interventionen, wie Aufzeichnung von Vitalparametern, bei Erwachsenen regelmäßiger erfolgten, da die Sicherheit der Rettungsassistenten bei der Erfassung der Parameter mit Zunahme des Alters der Patienten ansteigt (Gausche et al. 1990).

Die Ursache für die relativ seltene Anwendung invasiver Interventionen liegt einerseits in den eher niedrigen Schweregraden der meisten kindlichen Notfälle, andererseits in der mangelnden Toleranz der kleinen Patienten vor allem bei invasiveren Methoden. Weiterhin wurde von den Notärzten vermutlich besonders sorgsam abgewogen, ob das Kind einer invasiven Methode bedurfte oder nicht, sodass es bei steigendem Schweregrad der Verletzung oder Erkrankung durchaus zu einer Zunahme notwendiger invasiver Maßnahmen kam.

\subsection{Einschätzung von Diagnosen und Interventionen}

\subsubsection{Häufigkeit von Diagnosen und Interventionen}

Bei der Einschätzung der Häufigkeit von Diagnosen und therapeutischen Interventionen durch die befragten Notärzte lagen die Ergebnisse nahe an der Einsatzrealität im Göttinger Einsatzraum. Typische alterskorrelierte Krankheitsbilder wurden weitgehend erkannt, beispielsweise die Häufung von Krampfanfällen bei Kleinkindern und die mit dem kindlichen Alter zunehmende Inzidenz von Traumanotfällen (Eich et al. 2009a, Schlechtriemen et al. 2005a, Stenke 2004). In weitgehender Übereinstimmung mit der Literatur und mit den Göttinger Einsatzdaten schätzten die Notärzte auf einer Skala von 1 (sehr selten) bis 10 (sehr häufig) in der Altersgruppe ,Säuglinge“ die Diagnosen ,Krampfanfall` (inklusive Fieberkrampf), ,Pseudokrupp‘ sowie ,Verbrennung/Verbrühung‘ als häufigste Diagnosen ein, während Asthma bronchiale und Ertrinkungsunfälle als eher selten eingestuft wurden. In der Altersgruppe der Kleinkinder überwiegen nach Meinung der Befragten ebenfalls Pseudokrupp, Krampfanfall und Verbrennung/Verbrühung, während Atem- und Kreislaufstillstand sowie plötzlicher Kindstod (SIDS) als eher selten eingeschätzt wurden. Bei Schulkindern überwog laut der Befragung das Trauma als Notfalldiagnose in der Häufigkeit des Auftretens vor Asthma bronchiale und Schädel-Hirn-Trauma. 
Als relativ häufige Interventionen wurden in der Altersgruppe ,Säuglinge die intraossäre Punktion sowie die Intubation benannt, in der Altersgruppe der Kleinkinder ebenfalls Intubation und intraossäre Punktion in umgekehrter Reihenfolge und in der Altersgruppe der Schulkinder Intubation und Thoraxdrainage. Bei Kindern insgesamt selten erforderliche Interventionen wie beispielsweise die Thoraxdrainage wurden bezüglich ihrer Inzidenz korrekt eingestuft.

\subsubsection{Sicherheitsgefühl}

In der Befragung scheinen sich persönliche Erfahrungen aus der klinischen Anästhesie und Intensivmedizin widerzuspiegeln, einschließlich der bei Erwachsenen erworbenen Kenntnisse und Fertigkeiten. Laut Eich et al. hat die Mehrzahl der Anästhesisten in Deutschland nur eine sehr begrenzte kinderanästhesiologische Erfahrung. (Eich et al. 2006). Andererseits fühlten sich die Notärzte bei der endotrachealen Intubation, der Defibrillation sowie der kardiopulmonalen Reanimation vergleichsweise sicher, was vermutlich ihre Erfahrung aus der Erwachsenenmedizin widerspiegelt. Dies stimmt mit Ergebnissen aus der Untersuchung Bartels überein (Bartels 2001). Der Vergleich mit den eigenen Göttinger Einsatzzahlen zeigt, dass das empfundene Sicherheitsgefühl kaum aus der präklinischen Tätigkeit stammen kann, da gemäß der Auswertung der RED ${ }^{\circledR}$-Datenbank auch diese Interventionen bei Kindern eher selten angewandt werden (Eich et al. 2009a). Diese Erkenntnis bestätigt eine Analyse von Gries et al., in der bei fast 130.000 präklinischen Notfalleinsätzen die relative Seltenheit invasiver Interventionen mit der großen Anzahl tätiger Notärzte korreliert wurde (Gries et al. 2005).

Weiterhin wird aus der Befragung der aktiven Notärzte in Göttingen deutlich, dass bei als besonders selten eingestuften beziehungsweise klinisch selten anzutreffenden Diagnosen und Interventionen, wie beispielsweise der Epiglottitis oder der Einlage einer Thoraxdrainage, das persönliche Sicherheitsempfinden eher gering ist. Dagegen werden Diagnosen, die von den Befragten als häufig eingeschätzt wurden, mit einem entsprechend hohem Grad an Sicherheitsgefühl bewertet. So fühlten sich die von uns befragten Notärztinnen und Notärzte relativ sicher im Umgang mit den Diagnosen Krampfanfall, Pseudokrupp und Asthma in den Altersgruppen Säuglinge und Kleinkinder sowie im Umgang mit den Diagnosen Krampfanfall, Asthma bronchiale und Trauma in der Altersgruppe der Schulkinder. Vergleichbare Ergebnisse finden sich in der Untersuchung von Bartels (2001). 


\subsection{Ausbildungs- und Erfahrungsdefizite}

Nur die kontinuierliche praktische Übung scheint die erfolgreiche und sichere Durchführung von Notfallmaßnahmen bei Kindern zu gewährleisten, da erlernte Fähigkeiten bei nur sporadischer Anwendung nicht erhalten bleiben (Foltin 1995, Ballnus et al. 2002). Schon 2001 stellte Bartels in der Auswertung einer Umfrage unter Mitarbeitern des Rettungsdienstes im gesamten Bundesgebiet fest, dass Unsicherheiten bei der Versorgung von pädiatrischen Notfällen bei vielen Rettungsdienstmitarbeitern bestehen und lediglich 12 \% der befragten Ärzte bzw. 20 \% der befragten im Rettungsdienst tätigen Nichtärzte das bestehende Aus- und Weiterbildungsangebot als ausreichend erachten. Es darf daher die Frage gestellt werden, ob die gebotenen Möglichkeiten zur Ausbildung, vor allem im Bereich der praktischen Fähigkeiten und Fertigkeiten bei Kindern ausreichend sind, oder ob eine grundsätzliche Umstellung der Ausbildungskonzepte im Sinne der Patienten notwendig ist (Bartels 2001).

Bisher haben sich in Deutschland vor allem folgende Kurse zur Vertiefung kindernotfallmedizinischen Wissens und entsprechender Fertigkeiten etabliert:

- European Paediatric Life Support (EPLS) Kurs (ERC)

- Newborn Life Support (NLS) Kurs (ERC)

- Notfallmedizinisches Curriculum Kinderheilkunde (Deutsche Interdisziplinäre Vereinigung für Intensiv- und Notfallmedizin, DIVI)

- Kindersimulationsbasierte Kurse (Internationale PaedSim-Gruppe)

- Notarzt-Refresherkurse (Regionale Anbieter).

Der EPLS-Kurs des ERC, welcher regelmäßig auch vom Zentrum Anaesthesiologie, Rettungs- und Intensivmedizin der Universitätsmedizin Göttingen angeboten wird, ist ein standardisierter europäischer Intensivkurs, der sich an Ärzte, Funktionspflegepersonal sowie Rettungsassistenten richtet, die im Rahmen ihrer klinischen Tätigkeit mit der notfallmedizinischen Versorgung von Kindern betraut sind. Das Ziel dieses Kurses ist es, Wissen und Fertigkeiten für das Management des kritisch kranken Kindes in den ersten Stunden der Krankheit zu vermitteln und der Progression von Krankheiten bis zum kardialen Arrest vorzubeugen (Baskett et al. 2005). An drei Tagen werden Theorie und insbesondere Praxis der Basis- und der erweiterten Maßnahmen der kardiopulmonalen Reanimation (CPR) von Säuglingen und Kindern (Basic und Advanced Paediatric Life Support) nach den aktuellen Leitlinien des European Resuscitation Council (ERC), einschließlich der Erkennung und Versorgung vital gefährdeter Kinder, des Atemwegsmanagements, der Akutbehandlung lebens- 
bedrohlicher Herzrhythmusstörungen, der pädiatrischen Traumaversorgung und der Reanimation Neugeborener vermittelt. Dabei basiert der Kurs, neben Kurzvorträgen, vor allem auf praktischen Übungen in Kleingruppen. Ein besonderer Schwerpunkt liegt in der Simulation und Diskussion praxisnaher Szenarien der pädiatrischen Notfallversorgung, verbunden mit der Einübung eines an Algorithmen orientierten Handelns.

Der NLS-Kurs des ERC ist ein standardisierter eintägiger Intensivkurs, welcher die Maßnahmen zur Erstversorgung und zur Wiederbelebung eines Neugeborenen vermittelt. Er spricht alle an, die mit der Versorgung Neugeborener - wenn auch nur gelegentlich - konfrontiert sind. Es werden Wiederbelebungsmaßnahmen, ERC-Algorithmen sowie die Grundversorgung und die Schaffung von Zugangswegen beim Neugeborenen erlernt.

Das zweitägige „Notfallmedizinische Curriculum Kinderheilkunde“ wurde von der Deutschen Interdisziplinären Vereinigung für Intensiv- und Notfallmedizin (DIVI) erarbeitet (DIVI Stand April 2007, www.divi-org.de). Es beinhaltet den Umgang mit den wesentlichen Notfallsituationen bei Kindern, z. B. Reanimation, respiratorische Notfälle, Schock, Trauma, ZNS-Notfall, Intoxikation. In diesem Curriculum stellt die praktische Übung ein wesentliches Kursmodul dar.

Standardisierte, kindersimulationsbasierte Kurse (PaedSim-Kurse) sind inzwischen in Deutschland, Österreich und der Schweiz etabliert und gelten als dritte Stufe eines abgestuften Kursprogramms für kindliche Notfälle: 1. Wissen, 2. Fertigkeiten, 3. Einstellung und Verhalten (Umsetzung in die Praxis). Idealerweise sollen sich die Teilnehmer von PaedSimKursen durch Absolvierung strukturierter Vorbereitungskurse (EPLS oder ähnliche) gezielt vorbereiten, um dadurch Effektivität und Nachhaltigkeit des Trainings zu erhöhen. PaedSimKurse behandeln neben den medizinischen Aspekten („Technische Fertigkeiten“) kindlicher Notfälle zusätzlich Kriterien des Zwischenfallsmanagements (Crisis Resource Management, CRM). Letztere werden häufig auch als „nichttechnische Fertigkeiten“ bezeichnet (Fletcher et al. 2003). Somit liegt ein besonderer Schwerpunkt der PaedSim-Kurse auf der Übung von Kommunikation und Teamwork beim Kindernotfall (Heinzel 2008).

Notarzt-Refresherkurse richten sich an Ärzte, die vor längerer Zeit bereits die Zusatzqualifikation Notfallmedizin (bzw. den Fachkundenachweis Rettungsmedizin) erworben haben und in komprimierter Form ihr Wissen aktualisieren und ihre Fertigkeiten verbessern wollen. Diese Kurse unterschiedlicher Anbieter und Struktur sollen die teilnehmenden Notärzte also auf den Leistungsstand bringen, der heute nach Meinung der maßgeblichen Fachgesellschaften erwartet werden muss. 
Die objektiv selten auftretende kardiopulmonale Reanimation und Versorgung des polytraumatisierten Kindes, bei deren Umgang sich die Göttinger Notärzte unsicher fühlten, sind gekennzeichnet durch die perakute Vitalbedrohung des Kindes (je kränker und seltener, desto größer die Defizite).

Zum Zeitpunkt der Befragung hatte etwa ein Drittel der teilnehmenden Notärzte bereits einen speziellen Kindernotfallkurs (im wesentlichen EPLS-, NLS-Kurs, Curriculum Kinderheilkunde, PaedSim-Training, Notarztrefresherkurs) absolviert und war damit offensichtlich bestrebt, empfundene Defizite zu verringern. Allerdings sehen bereits in der Umfrage von Bartels 88 \% der befragten Ärzte das Ausbildungsangebot für präklinische Kindernotfälle als nicht ausreichend an (Bartels 2001).

In unserer Untersuchung wurden viele Verbesserungsvorschläge zur Optimierung der Ausbildung bezüglich der präklinischen Kindernotfallmedizin gemacht. Ähnlich wie in der Befragung durch Bartels 2001 wurden beispielsweise die Möglichkeit zur Hospitation in der Kinderklinik bzw. Kinderintensivabteilung, in der Kinderanästhesie und im Baby-Notarztwagen empfohlen, ebenso die Vorbereitung auf psychisch belastende Einsätze und der Wunsch nach einer praxisnäheren Ausbildung geäußert. In Göttingen war zudem das simulatorbasierte Training ein besonders häufig vorgebrachter Vorschlag zur Verbesserung von Aus-, Weiter- und Fortbildung in der Kindernotfallmedizin. Die befragten Notärzte wollten vor allem die Notfallsituationen, bei denen sie sich unsicher fühlen, am Patientensimulator trainieren. Dazu zählen u. a. die kardiopulmonale Reanimation, das Atemwegsmanagement und die (Poly-)Traumaversorgung.

In diesem Sinne wurde von zahlreichen Notärzten auch eine intensivere Einbeziehung in die klinische Versorgung kranker Kinder im Rahmen von Kinderintensivmedizin und Kinderanästhesie gewünscht. Darüber hinaus wurde zudem von mehreren Kollegen eine regelmäßige Notarzt-Rezertifizierung gefordert, bei der speziell auch kindernotfallmedizinische Aspekte besondere Berücksichtigung finden sollten.

\subsection{Simulatorbasierte Lehre}

Ein Vorteil der Simulation, einer in der Luftfahrt bereits seit Jahrzehnten etablierten Ausbildungsform, liegt vor allem im sicheren und reproduzierbaren Training (Mönk 2003). Auch in anderen Untersuchungen fordert man eine praxisorientiertere Ausbildung in der Notfallmedizin wie die Einbeziehung von simulationsunterstützter Fortbildung, die sich weltweit mehr und mehr etabliert (Eich et al. 2006, Eppich et al. 2006, Gräsner et al. 2006, Gries et al. 2003, 
Gries und Kreimeier 2008, Issenberg et al. 1999, Seidel 1986, Timmermann et al. 2005, Timmermann et al. 2007). Die simulatorbasierte Lehre unterstützt dabei gezielt die einzelnen Ebenen der Lernpyramide nach Miller:

1. Knows (Vermittlung von Wissen und Fertigkeiten);

2. Knows how (Demonstration von Wissen und Fertigkeiten);

3. Shows how (Anwendung von Wissen und Fertigkeiten);

4. Does (praktische Umsetzung innerhalb eines Szenarios

(Miller 1990, Timmermann et al. 2007).

Dadurch werden theoretisches Wissen und praktisch-klinische Fertigkeiten besser in die spätere klinische Anwendung transferiert (Timmermann et al. 2007). Bisher wurden vor allem technische Fertigkeiten an Übungsphantomen trainiert. In der modernen simulatorbasierten Lehre wird ein großer Schwerpunkt auf die interpersonelle Interaktion gelegt: Wie kommuniziere ich effektiv? Wie arbeite ich im Team? Wie nutze ich mir zur Verfügung stehende Ressourcen? Auch für ein Training in der Kinderanästhesie und Kindernotfallmedizin stehen integrierte Säuglingssimulatoren zur Verfügung (Eich et al. 2006).

Unter Berücksichtigung der detaillierten Angaben zu speziellen Trainingswünschen am Kindersimulator ergibt sich, dass vor allem Notfälle trainiert werden sollten, denen man im klinischen Alltag gar nicht oder eher selten begegnet. Aber auch der Umgang mit Patienten und dem Team sowie mit Stresssituationen oder Krisenmanagement sollte geübt und verinnerlicht werden (Crisis Resource Management) (Baskett et al. 2005, Eppich et al. 2006, Mönk 2003, Timmermann et al. 2005, Yee et al. 2005). Gerade in diesem Bereich liegen die besonderen Stärken des simulatorbasierten Trainings, einschließlich der realistischen Simulation des oft ungewohnten Umgangs mit den anwesenden Eltern bei kindlichen Notfällen (Wantman und Chin 2003).

Während jedoch die technischen Möglichkeiten der Kindersimulation bereits gut entwickelt sind, gibt es noch immer erhebliche organisatorische Defizite bezüglich einer strukturierten und formalen Etablierung (Eich et al. 2006, Eich et al. 2007b). Daher wurde in Deutschland, Österreich und der Schweiz 2008 eine interdisziplinäre Arbeitsgruppe gegründet, welche die Etablierung eines standardisierten Kindersimulationskurses (PaedSim) für Kinderanästhesie, Kinderintensiv- und Kindernotfallmedizin koordiniert (www.simulation.ch/ Kurse/Aerzte/Kursprogramm/Foramte/PaedSim). Dabei soll das kindersimulationsbasierte Training durch eine festgelegte Teilnahmevoraussetzung (z. B. EPLS-Zertifikat) sowie durch ein einheitliches, strukturiertes Curriculum und durch eine formale Zertifizierung von Seiten 
der zuständigen Fachgesellschaften in seiner Effektivität und Nachhaltigkeit optimiert werden.

Das Training am Patientensimulator kann Lernen und Erfahrungen am Krankenbett nicht ersetzen, diese jedoch gezielt, wirkungsvoll und nachhaltig ergänzen (Eich et al. 2006, Eppich et al. 2006). Timmermann et al. kommen zu dem Schluss, das simulatorunterstütztes Training einen Beitrag dazu leistet, die Effektivität der Maßnahmen beim Einsatz am Patienten zu erhöhen, Akzeptanz und Sicherheit der Verfahren beim Anwender zu steigern und damit für mehr Patientensicherheit zu sorgen (Timmermann et al. 2005).

\subsection{Limitationen}

Bei allen Vorteilen einer Eincenterstudie (vollständige Datensammlung, hohe Rücklaufrate der Fragebögen) ergeben sich nichtsdestotrotz potenzielle Limitationen. So lassen sich die Ergebnisse unserer Umfrage und der retrospektiven Auswertung der RED ${ }^{\circledR}$-Datenbank möglicherweise nur eingeschränkt auf andere Rettungsdienstbereiche und Notarztpopulationen übertragen, da die befragten Göttinger Notärzte durch ihre Erfahrung in der Kinderanästhesie im Vergleich zur Gesamtheit der deutschen Notärzte eine relativ hohe Expertise bei der Versorgung kritisch kranker Kinder besitzen. In diesem Zusammenhang wäre es interessant zu wissen, wie Notärzte anderer Disziplinen ohne innerklinischen Kontakt zu kranken Kindern präklinische Kindernotfälle wahrnehmen und einschätzen und wie sich die Verteilung der Häufigkeiten von Diagnosen in anderen Gebieten verhält.

\subsection{Schlussfolgerungen und Ausblick}

Auch wenn pädiatrische Notfälle seltener auftreten als bei erwachsenen Patienten, muss jeder Notarzt sicher im Umgang mit Kindernotfällen sein. Somit ist eine fundierte Aus-, Weiterund Fortbildung im Umgang mit pädiatrischen Notfällen sinnvoll und zwingend, zumal sich der Notarzt aufgrund des äußeren Drucks in einer besonders anspruchsvollen Situation befindet. Die bisher unzureichenden Aus-, Weiter- und Fortbildungsmöglichkeiten der aktiven Notärzte müssen hinterfragt werden. Wichtig erscheint der Ausbau der aktuellen Trainingsmöglichkeiten vor allem hinsichtlich der weitergehenden Nutzung der simulatorbasierten Lehre zur Steigerung des Sicherheitsgefühls der Notärzte im Umgang mit dem pädiatrischen Patienten. Die sich daraus ergebenden finanziellen und organisatorischen Probleme müssen ausgewertet und gelöst werden. Zusammenschlüsse zur Organisation der Lehre im Sinne von 
PaedSim, einem interdisziplinären Kooperationsprojekt von Simulationszentren in Deutschland der Schweiz und Österreich, schaffen Synergieeffekte zur Etablierung dieser Form der Lehre in der (notfall-)medizinischen Aus-, Weiter- und Fortbildung. Die simulatorbasierte Lehre ist bereits fester Bestandteil der Weiterbildung im Zentrum Anaesthesiologie, Rettungsund Intensivmedizin der Universitätsmedizin Göttingen. 


\section{Zusammenfassung}

Präklinische Kindernotfälle haben im Vergleich zu Notfällen im Erwachsenenalter eine niedrigere Inzidenz. In Deutschland werden präklinische Kindernotfälle überwiegend von nichtspezialisierten Notärzten unterschiedlicher Fachrichtungen versorgt. In der Regel haben diese eine relativ geringe Routine im Umgang mit Kindernotfällen, da die Berührungspunkte in der klinischen Versorgung oft gering sind. Geringe Inzidenz und Routine führen häufig zu Angst und Unsicherheit im Umgang mit pädiatrischen Notfallpatienten. Unklar war bisher, welcher Natur die Ängste sind und in welchem Zusammenhang sie mit eigenen Wahrnehmungen und Einschätzungen stehen und wie sie mit realen Einsatzdaten im Rettungsdienst korrelieren.

Es wurde ein strukturierter Fragebogen zu Wahrnehmung und Einschätzung präklinischer Kindernotfälle erstellt und an die im Untersuchungszeitraum tätigen Notärzte des Zentrums für Anaesthesiologie und Intensivmedizin der Universitätsmedizin Göttingen ausgegeben. Weiterhin werteten wir retrospektiv alle innerhalb eines Neun-Jahres-Zeitraumes erfassten präklinischen Kindernotfälle in der Luft- und Bodenrettung unseres Zentrums aus.

43 Notärzte nahmen an der Befragung teil. Es wurden sehr differenzierte Angaben zu den erwarteten Charakteristika präklinischer Kindernotfälle gemacht. Tendenziell zeigte sich ein Zuwachs des Sicherheitsempfindens in Abhängigkeit mit dem Alter des Patienten sowie mit der eigenen Expertise. Deutliche Erfahrungsdefizite wurden in der kardiopulmonalen Reanimation und der Traumaversorgung gesehen. Die Einschätzung der Inzidenz ausgewählter Diagnosen und therapeutischer Interventionen zeigte sich in guter Relation zur Realität. Die Befragten favorisierten Kindersimulatortraining und Praktika in der Kinderintensivmedizin als Fortbildungsstrategien.

Im Göttinger Raum wurden 2.271 pädiatrische präklinische Kindernotfälle im Zeitraum vom 1.Januar 1998 bis 31.Dezember 2006 identifiziert (6,3\% Anteil am Gesamteinsatzaufkommen, 8,5 \% am Gesamteinsatzaufkommen im Helikopter). 72,7 \% dieser Notfälle waren weniger schwerwiegend (NACA I-III), 27,3 \% waren von höherem Schweregrad (NACA IV-VII), im luftgebundenen Rettungsdienst NACA I-III $68 \%$ und NACA IV-VII 32 \%. Die häufigsten Notfalldiagnosen waren respiratorische Störungen (in der Altersgruppe der Säuglinge 34,5 \%, in der Altersgruppe der Kleinkinder 21,8 \%, in der Altersgruppe der Schulkinder15,0 \%), Krampfanfälle inklusive Fieberkrampf (Säuglinge: 17,2 \%, Kleinkinder: 43,2 \%, Schulkinder: 16,0 \%) sowie Trauma (Säuglinge: 16,0 \%, Kleinkinder: 19,5 \%, Schulkinder: 44,4\%). 
Endotracheale Intubation wurde in 4,2 \% der Notfälle angewendet (im luftgebundenen Rettungsdienst in 7,6 \% der versorgten Notfälle), die intraossäre Punktion in 0,7\% der Kindernotfälle (im luftgebundenen Rettungsdienst 1,0\%). Eine kardiopulmonale Reanimation wurde bei 2,3 \% der Kindernotfälle (in der Luftrettung in 3,4 \% der Fälle) begonnen. Thoraxdrainage, Thorakozentese und Defibrillation fanden im Untersuchungszeitraum selten Anwendung. Im luftgebundenen Rettungsdienst findet sich ein deutlich höherer Anteil von Ertrinkungsunfällen (80 \%), Kopfverletzungen (inklusive SHT) (73,9\%) und SIDS (60,0 \%), während 75 \% der respiratorischen Störungen durch den bodengebundenen Rettungsdienst versorgt wurden.

Die Wahrnehmung und Einschätzung der anästhesiologischen Notärzte korrelierte überwiegend in hohem Maße mit der Realität. Die größten Erfahrungsdefizite wurden im Bereich seltener, in der Regel vitalbedrohlicher Notfälle erkannt.

Diese Untersuchung zeigt auf, dass deutsche Notärzte vor allem im Umgang mit häufigen pädiatrischen Diagnosen wie respiratorischen Störungen, Krampfanfällen und Traumata besonders gut geschult sein müssen. Auch wenn vitalbedrohliche Kindernotfälle, bei denen häufig therapeutische Interventionen angewendet werden, eher in der Luft- als in der Bodenrettung auftreten, sind profunde Kenntnisse im Umgang mit Diagnosen und Interventionen wie kardiopulmonaler Reanimation und intraossärer Punktion essenziell in der Ausbildung des interdisziplinären Notarztes.

Insgesamt können drei wesentliche Lernstrategien manifestiert werden, die in der Ausbildung des Notarztes Berücksichtigung finden sollten:

1. Kenntnisse und Fertigkeiten, die innerklinisch, im Rahmen von Hospitationen/Rotationen erlernt werden können;

2. die beim Erwachsenen erworbene Expertise, die sich zum Teil auf den pädiatrischen Patienten übertragen lässt, vor allem bezüglich Patienten im Schulkindalter;

3. Krankheitsbilder und therapeutische Interventionen, die aufgrund ihrer Seltenheit fast ausschließlich am Phantom oder Kindersimulator trainiert werden können, um das Sicherheitsgefühl der behandelnden Notärzte im Falle eines Falles zu erhöhen und so Behandlungsfehler zu verringern und die präklinische Versorgung des pädiatrischen Patienten zu optimieren. 


\section{Anhang \\ 1. Fragebogen}

\section{Georg-August-Universität Göttingen}

\section{BEREICH HUMANMEDIZIN / UNIVERSITÄTSKLINIKUM \\ ZENTRUM ANAESTHESIOLOGIE, RETTUNGS- U. INTENSIVMEDI- ZIN}

Direktoren: Prof. Dr. med. Bernhard M. Graf

Prof. Dr. med. Michael Quintel

\section{FRAGEBOGEN „PRÄKLINISCHE KINDERNOTFÄLLE“}

Liebe Kollegin, lieber Kollege,

im Rahmen einer Studie zur Ausbildung in der Notfallmedizin bitten wir Sie um Ihre Teilnahme an einer kurzen Befragung. Diese umfasst das Ausfüllen des folgenden kurzen Fragebogens und dessen Rücksendung an ceich@zari.de oder als Papierausdruck in das Postfach von Christoph Eich.

Herzlichen Dank

für Ihre Mitwirkung!

Christoph Eich

Oliver Radke

Markus Roessler

Fragen zu Person, Ausbildung und notfallmedizinischer Erfahrung:
$1 \quad$ Ihr Alter?
$\square$ 25-30
$\square 31-40$
$\square 41-50$
$\square$ 51-60
$\square>60$ Jahre

$\square \mathrm{W}$

$\square \mathrm{m}$

3a Ihre Fachrichtung?

3b Ihr Status in dieser Fachrichtung?

$\square$ in Weiterbildung im _ ten Jahr $\square$ Fachärztin/-arzt wenn, ja seit wann?

$4 \quad$ In welchem medizinischem Umfeld Sie derzeit tätig?
$\square \mathrm{KH}$ der Grund-/Regelversorgung
$\square$ Universitätsklinik
$\square \mathrm{KH}$ der Schwerpunkt-/Maximalversorgung
$\square$ Hauptberufliche/r Notärztin/-arzt
$\square$ Praxis
$\square$ anderes

5a Sind Sie in Ihrer klinischen Routine in die Versorgung von Kindern involviert?

$$
\square \text { ja } \square \text { nein }
$$

5b Wenn ja, im welchem/n Bereich/en? 
6a Besitzen Sie die Fachkunde bzw. Zusatzbezeichnung Rettungs-/Notfallmedizin? $\square$ ja $\square$ nein

6b Wenn ja, erworben am bei der Ärztekammer

7a Sind Sie tätig als Notarzt/-ärztin? $\square$ ja $\square$ nein

7b Wenn ja, seit $\quad \square<1$ Jahr $\square$ 1-2 $\square$ 3-5 $\square$ 5-10 $\square>10$ Jahren

8 Wie hoch ist die Zahl Ihrer Notarzteinsätze pro Jahr (im Mittel der letzten drei Jahre)?

9 Auf welchen Rettungsmitteln sind/waren Sie tätig? $\square$ NEF/NAW $\square$ RTH 
Fragen zu Kindernotfällen:

10a Wie hoch schätzen Sie den Anteil der notärztlichen Einsätze mit Kindern (unter 15 Jahren) im Rettungsdienstbereich Göttingen ein (ca. 4200 Notarzteinsätze/Jahr insgesamt)?
$\square<1 \%$
$\square 1-3 \%$
$\square 3,1-6 \%$
$\square 6,1-10 \%$
$\square 10,1-15 \% \quad \square>15 \%$

$10 \mathrm{~b}$ davon Säuglinge ( $<1$ Jahr $)$

$$
\begin{aligned}
& \hline<10 \% \\
& \square 11-30 \% \\
& \square 31-50 \% \\
& \square>50 \%
\end{aligned}
$$

\author{
Kleinkinder 1-5 Jahre \\ $\square<10 \%$ \\ $\square 11-30 \%$ \\ $\square 31-50 \%$ \\ $\square>50 \%$
}

Schulkinder 6-14 Jahre

$\square<10 \%$

$\square 11-30 \%$

$\square 31-50 \%$

$\square>50 \%$

11 Den Schweregrad von Kindernotfällen (unter 15 Jahren) im Rettungsdienstbereich Göttingen betreffend: Wie hoch schätzen Sie den Anteil der Altersgruppen an den NACA-Graden I-III bzw. IV-VII ein?
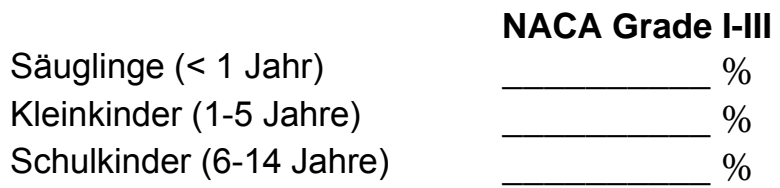

NACA (National Advisory Committee for Aeronautics)

$\begin{array}{ll}\text { I: } & \text { Geringfügige Störung } \\ \text { II: } & \text { Ambulante Abklärung } \\ \text { III: } & \text { Stationäre Behandlung }\end{array}$

12 Wie hoch schätzen Sie die Häufigkeit folgender Verdachtsdiagnosen bei Kindern im Rettungsdienstbereich Göttingen ein? (zutreffendes bitte markieren)

$$
\text { Säuglinge (< } 1 \mathrm{~J} .) \quad \text { Kleinkinder (1-5 J.) Schulkinder (6-14 J.) }
$$

1 sehr selten sehr häufig 10

$\begin{array}{lllll}\text { Atem- u/o Kreislaufstillstand } & 12345678910 & 12345678910 & 12345678910 \\ \text { Plötzlicher Kindstod (SIDS) } & 12345678910 & 12345678910 & 12345678910 \\ \text { Ertrinkungsunfall } & 12345678910 & 12345678910 & 12345678910 \\ \text { Verbrennung/Verbrühung } & 12345678910 & 12345678910 & 12345678910 \\ \text { Pseudokrupp } & 12345678910 & 12345678910 & 12345678910 \\ \text { Epiglottitis } & 12345678910 & 12345678910 & 12345678910 \\ \text { Asthma bronchiale } & 12345678910 & 12345678910 & 12345678910 \\ \text { Trauma } & 12345678910 & 12345678910 & 12345678910 \\ \text { Schädel-Hirn-Trauma } & 12345678910 & 12345678910 & 12345678910 \\ \text { Krampfanfall } & 12345678910 & 12345678910 & 12345678910 \\ \text { (einschl. Fieberkrampf) } & 123456\end{array}$
dienstbereichGöttingen ein? (zutreffendes bitte markieren)

\begin{tabular}{ccc} 
Säuglinge (<1 J.) & Kleinkinder (1-5 J.) & Schulkinder (6-14 J.) \\
1 sehr selten & sehr häufig 10 & \\
\hline 12345678910 & 12345678910 & 12345678910 \\
12345678910 & 12345678910 & 12345678910 \\
12345678910 & 12345678910 & 12345678910 \\
12345678910 & 12345678910 & 12345678910
\end{tabular}




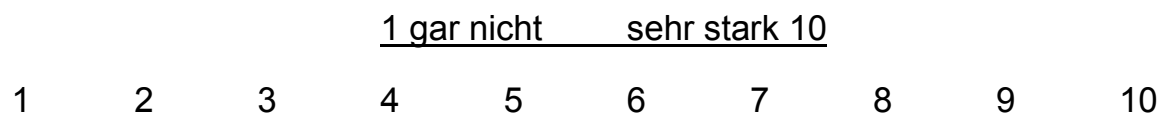

16 Wie sicher fühlen Sie sich bei folgenden präklinischen Notfällen bzw. Interventionen bei Kindern? (zutreffendes bitte markieren)

$$
\text { Säuglinge (< } 1 \text { J.) Kleinkinder (1-5 J.) Schulkinder (6-14 J.) }
$$

1 sehr unsicher sehr sicher 10

$\begin{array}{lllll}\text { Atem- u/o Kreislaufstillstand } & 12345678910 & 12345678910 & 12345678910 \\ \text { Plötzlicher Kindstod (SIDS) } & 12345678910 & 12345678910 & 12345678910 \\ \text { Ertrinkungsunfall } & 12345678910 & 12345678910 & 12345678910 \\ \text { Verbrennung/Verbrühung } & 12345678910 & 12345678910 & 12345678910 \\ \text { Pseudokrupp } & 12345678910 & 12345678910 & 12345678910 \\ \text { Epiglottitis } & 12345678910 & 12345678910 & 12345678910 \\ \text { Asthma bronchiale } & 12345678910 & 12345678910 & 12345678910 \\ \text { Trauma } & 12345678910 & 12345678910 & 1234567910 \\ \text { Schädel-Hirn-Trauma } & 12345678910 & 12345678910 & 12345678910 \\ \text { Krampfanfall } & 12345678910 & 12345678910 & 12345678910 \\ \text { (einschl. Fieberkrampf) } & 12345678910 & 12345678910 & 12345678910 \\ \text { Intubation } & 12345678910 & 12345678910 & 12345678910 \\ \text { Intraossäre Punktion } & 12345678910 & 12345678910 & 12345678910 \\ \text { Defibrillation } & 12345678910 & 12345678910 & 12345678910\end{array}$

17 Wie sicher fühlen Sie sich in der notärztlichen Versorgung folgender Altersgruppen? (Zutreffendes bitte markieren)

1 sehr unsicher $\quad$ sehr sicher 10

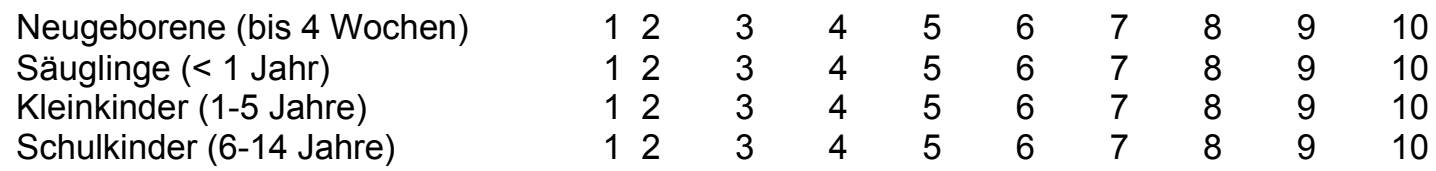


18 Auf welche kindlichen Notfälle fühlen Sie sich unzureichend vorbereitet?

20 Wie könnte Ihrer Meinung nach die Ausbildung in der Kindernotfallmedizin verbessert werden?

21 Welche Notfälle würden Sie gerne am Phantom bzw. mit Hilfe eines KinderISäuglingssimulators trainieren?

[Fragebogen „Präklinische Kindernotfälle“ - CEich et al. 2005] 


\section{Literatur}

ÄK Niedersachsen 2004

Aijin et al. 1989

Albrech et al. 2000

Altemeyer et al. 2000

Applebaum 1985

Atkins et al. 2009

Babl et al. 2001

BÄK 2004

Ballnus et al. 2002

Bartels 2001

Baskett et al. 2005

Bernhard et al. 2006
Ärztekammer Niedersachsen Weiterbildungsordnung Notfallmedizin: www.aerztekammer-niedersachsen.de vom 27.11.2004

Aijin P, Tsai A, Knopp R, Kallsen GW (1989): Endotracheal Intubation of Pediatric Patients by Paramedics.

Ann Emerg Med, 18, 489-494

Albrech M, Berner J, Altemeyer KH (2000): Kindernotfälle im Luftrettungsdienst.

Notfall Rettungsmed, $\underline{3}, 156-169$

Altemeyer KH, Schlechtriemen T, Albrech M (2000): Notfälle im Kindesalter - Beispiele aus der präklinischen Erstversorgung.

Notfall Rettungsmed, $\underline{3}$, 186-198

Applebaum D (1985): Advanced Prehospital Care for Pediatric Emergencies Ann Emerg Med, 14, 656-659

Atkins DL, Everson-Stewart S, Sears GK, Daya M, Osmond MH, Warden CR, Berg RA (2009): Epidemiology and Outcomes from Out-of-Hosptal Cardiac Arrest in Children: The ROC Epistry-Cardiac Arrest

Circulation, 119, 1484-1491

Babl FE, Vinci RJ, Bauchner H, Mottley L (2001): Pediatric pre-hospital advanced life support care in an urban setting.

Pediatr Emerg Care, 17, 5-9

Bundesärztekammer Curriculum Notfallmedizin:

www.bundesärztekammer.de 2004

Ballnus S, Möller JC, Friedrich HJ (2002): Evaluation der dokumentierten Versorgung von Kindern im Einzugsgebiet des Universitätsklinikums Lübeck.

Notarzt, $\underline{18}, 187-191$

Bartels, U. (2001): Kindernotfälle.

Notarzt, 17, 31-36

Baskett PJF, Nolan JP, Handley A, Soar J, Biarent D, Richmond S (2005):

European Resuscitation Council Guidelines for Resuscitation 2005 Section 9. Principles of training in resuscitation.

Resuscitation, 67 Suppl, 181-189

Bernhard M, Hilger T, Sikinger M, Hainer C, Haag S, Streitberger K, Martin E, Gries A (2006): Patientenspektrum im Notarztdienst. Was hat sich in den letzten 20 Jahren geändert?

Anaesthesist, 포, 1157-1165 
Bernhard et al. 2008

Biarent et al. 2005

Blanco-Ons Fernández et al. 2007

Boswell et al. 1995

Brambrink 1998

Brambrink 1999

Brambrink 2002

Brownstein et al. 1996

Burghofer und Lackner 2006

Chen und Hsiao 2008

Committee on Pediatric Emergency

Medicine 2007

DiRusso et al. 2005

DIVI 2007
Bernhard M, Aul A, Helm M, Mutzbauer TS, Kirsch J, Brenner T, Hainer C, Gries A (2008): Invasive Notfalltechniken in der Notfallmedizin - Indikationen und Ausbildungskonzepte.

Notfall Rettungsmed, 11, 304-309

Biarent et al, European Rescucitation Council guidelines for rescucitation 2005. Section 6. Paediatric life support.

Rescucitation 2005: 67 Suppl 1: S. 97-133

Blanco-Ons Fernández P, Sánchez-Santos L, Rodríguez-Núñez IglesiasVásquez JA, Cegarra-García M, Barreiro-Díaz, M (2007): Paediatric out-ofhospital resuscitation in an area with scattered population (Galicia-Spain).

BMC Emerg Med, 7, 1-6

Boswell WC, McElveen N, Sharp M, Boyd CR, Frantz EI (1995): Analysis of Prehospital Pediatric and Adult Intubation.

Air Med J, 14, 125-128

Brambrink AM (1998): Eine neue Rubrik stellt sich vor. Notfälle im Kindesalter - eine interdisziplinäre Herausforderung

Notfall Rettungsmed, 1 , 361-370

Brambrink AM (1999): Neue Hinweise zur Behandlung von Kindern in Notfallsituationen.

Notfall Rettungsmed, 2, 235-240

Brambrink AM (2002): Die Primärversorgung des kindlichen Schädel-HirnTraumas aus der Sicht des Notarztes.

Notfall Rettungsmed, $\underline{5}$, 332-334

Brownstein D, Shugerman R, Cummings P, Rivara F, Copass M (1996): Prehospital Endotracheal Intubation of children by Paramedics Ann Emerg Med, 28, 34-39

Burghofer K, Lackner CK (2006): Tun und Lassen in der Notfallmedizin Notfall Rettungsmed, $\underline{9}$, 685-690

Chen L, Hsiao AL (2008): Randomized Trial of Endotracheal Tube Versus Laryngeal Mask Airway in Simulated Prehospital Pediatric Arrest

Pediatrics, 122, e294-e297

Committee on Pediatric Emergency Medicine (2007): Preparation for Emergencies in the Offices of Pediatricians and Pediatric Primary Care Providers. Pediatrics, $\underline{120}$, 200-212

DiRusso SM, Sullivan T, Risucci D, Nealon P, Slim M (1995): Intubation of Pediatric Trauma Patients in the Field: Predictor of Negative Outcome Despite Risk Stratification.

J Trauma, 모, 84-91

Deutsche interdisziplinäre Vereinigung für Intensiv- und Notfallmedizin, www.divi.org.de 2007 
Donoghue et al. 2006

Easley et al. 2000

Eckstein et al. 2002

Eich et al. 2006

Eich et al. $2007 a$

Eich et al. $2007 b$

Eich et al. 2009a

Eich et al. $2009 b$

Eppich et al. 2006

Fifield et al. 1984

Flake und Heinichen 2010
Donoghue AJ, Nadkarni, VM, Elliott M, Durbin D (2006): Effect of Hospital Characteristics on Outcomes from Pediatric Cardiopulmonary Resuscitation: A Report from the National Registry of Cardiopulmonary Resuscitation Pediatrics, 118, 995-1001

Easley RB, Segeleon JE, Haun SE, Tobias JD (2000): Prospective study of airway management of children requiring endotracheal intubation before admission to a pediatric intensive care unit.

Crit Care Med, 28, 2058-2063

Eckstein M, Jantos T, Kelly N, Cardillo A (2002): Helicopter Transport of Pediatric Trauma Patients in an Urban Emergency Medical Services System: Aritical Analysis.

J Trauma, 프, 340-344

Eich C, Russo S, Timmermann A, Nickel EA, Graf BM (2006): Neue Perspektiven der simulatorgestützten Ausbildung in Kinderanästhesie und Kindernotfallmedizin

Anaesthesist, $\underline{55}, 179-184$

Eich C, Weiss M, Hüpfl M (2007): Lebensrettende Maßnahmen bei Kindern nach aktuellen Leitlinien. PLS - Wie wird's gemacht?

Notfall Rettungsmed, 10, 117-123

Eich C, Timmermann A, Russo SG, Nickel EA, McFadzean J, Rowney D, Schwarz SK (2007): Simulator based training in paediatric anaesthesia and emergency medicine-thrills, skills and attitudes.

Br J Anaesth, 98, 417-419

Eich C, Russo SG, Heuer JF, Timmermann A, Gentkow U, Quintel M, Roessler M (2009): Characteristics of out-of-hospital paediatric emergencies attended by ambulance- and helicopter-based emergency physicians

Resuscitation. 80, 888-892

Eich, C, Roessler, M, Nemeth, M, Russo, SG, Heuer, JF, Timmermann, A (2009): Characteristics and outcome of prehospital paediatric tracheal intubation attended by anaesthesia-trained emergency physicians

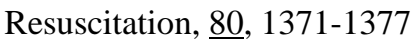

Eppich WJ, Adler MD, McGaghie WC (2006): Emergency and critical care pediatrics: use of medical simulation for training in acute pediatric emergencies.

Curr Opin Pediatr, 18, 266-271

Fifield GC, Magnuson C, Carr WP, Deinard AS (1984): Pediatric Emergency In a Metropolitan Area

J Emerg Med, 1, 495-507

Flake F, Heinichen F: Kindernotfälle im Rettungsdienst. 3. Auflage

Springer Medizin Verlag Heidelberg, 2007 
Fletcher et al. 2003

Foltin 1995

Francis et al. 2008

Gausche et al. 1990

Gausche et al. 2000

Gerlacher et al. 2001

Gervais 2001

Gortner 1998

Gräsner et al. 2006

Graham 2004

Graham et al. 1993

Gries und Wilhelm 2004
Fletcher G, Flin R, Mc George P, Glsvin R, Maran N, Paley R (2003): Anaesthesists' non technical skills (ANTS): evaluation of a behavioural marker system.

Br J Anaesth, 90, 580-588

Foltin GL (1995): Critical Issues in Urban Emergency Medical Services for Children.

Pediatrics, $\underline{96}, 174-179$

Francis RCE, Höhne C, Proquitte H, Kerner T (2008): Reanimation bei Kindern. Empfehlung nach aktuellen Leitlinien.

Notarzt, 24, 124-134

Gausche M, Henderson DP, Seidel JS (1990): Vital signs as parameter of the prehospital assessment of the pediatric patient: a survey of paramedics.

Ann Emerg Med, 19, 173-178

Gausche M, Lewis RJ, Stratton SJ (2000): Effect of Out -of -Hospital Pediatric Endotracheal Intubation on Survival and Neurological Outcome: A Controlled Clincal Trial.

JAMA, 283, 783-790

Gerlacher GR, Sirbaugh PE, Macias CG (2001): Prehospital evaluation of non-transported pediatric patients by a large emergency medical services system.

Pediatr Emerg Care, 17, 421-424

Gervais HW (2001): Reanimation im Neugeborenen- und Kindesalter.

Notfall Rettungsmed, 4 , 16-22

Gortner L (1998): Anatomische und physiologische Besonderheiten im Kindesalter

Notfall Rettungsmed, $\underline{1}$, 363-366

Gräsner JT, Bahr J, Böttiger BW, Cavus E, Dörges V, Gries A, Krieter H, Wenzel V, Scholz J (2006): Notfallmedizin in Ausbildung, Lehre, Qualitätsmanagement, Grundlagenforschung und in klinischen Studien - 2. Wissenschaftliches Treffen des AK Notfallmedizin der DGAI.

Notfall Rettungsmed, $\underline{9}$, 702-710

Graham CA (2004): Advanced airway management in the emergency department: what are the training and skills maintenance needs for UK emergency physicians?

Emerg Med J, 21, 14-19

Graham CJ, Stuemky J, Lera TA (1993): Emergency medical services preparedness for pediatric emergencies.

Pediatr Emerg Care, $\underline{9}$, 329-331

Gries A, Wilhelm W (2004): Defizite im präklinischen Management bestimmter Notfallsituationen.

Anaesthesist, 프, 1043-1044 
Gries und Kreimeier 2008

Gries et al. 2003

Gries et al. 2005

Heinzel 2008

Helm et al. 1999

Helm et al. 2005

Helm et al. 2007

Hirschfeld 1995

ILCOR 2005a

ILCOR 2005b

Issenberg et al. 1999

Jester et al. 2006

Jewkes 2001
Gries A, Kreimeier U (2008): invasive Notfalltechniken - Heute überhaupt noch notwendig oder wichtiger denn je?

Notfall Rettungsmed, $\underline{11}$, 302-303

Gries A, Helm M Martin E (2003): Zukunft der präklinischen Notfallmedizin in Deutschland.

Anaesthesist, 프, 718-724

Gries A, Zink W, Bernhard M, Messelken M, Schlechtriemen T (2005):

Einsatzrealität im Notarztdienst

Notfall Rettungsmed, $\underline{8}$, 391-398

Heinzel O(2008): Simulationstraining für kritische Ereignisse - eine innovative Ausbildungsform auch für die Pädiatrie.

Monatsschr Kinderheilkd, $\underline{156}, 426$

Helm M, Hauke J, Frey W, Lampl L (1999): Der pädiatrische Traumapatient im Luftrettungsdienst

Notfall Rettungsmed, 2, 150-157

Helm M, Gries A, Fischer S, Hauke J, Lampl L (2005): Invasive Techniken in der Notfallmedizin.

Anaesthesist, 54, 49-56

Helm M, Hauke J, Bippus N, Lampl L (2007): Die intraossäre Punktion in der präklinischen Notfallmedizin.

Anaesthesist, 트, 18-24

Hirschfeld JA (1995): Emergency medical Services for children in Rural and Frontier America: Diverse and Changing Environments.

Pediatrics, $\underline{96}$, 179-184

International Liaison Committee on Resuscitation (2005): Part 6: Paediatric basic and advanced life support

Resuscitation, 67, 271-291

International Liaison Committee on Resuscitation (2005): Part 7: Neonatal resuscitation

Resuscitation, 67, 293-303

Issenberg SB, McGaghie WC, Hart IR, Mayer JW, Felner JM, Petrusa ER, Waugh RA, Brown DD, Safford RR, Gessner IH, Gordon DL, Ewy GA (1999) : Simulation Technology for Health Care Professional Skills Training and Assessment

JAMA, 282, 861-866

Jester I, Genzwürker H, Jester A, Demirakca S, Waag KL (2006): Notfallmanagement bei kindlichen Verbrennungen.

Notfall Rettungsmed, $\underline{9}$, 227-238

Jewkes F (2001): Current topic: Prehospital emergency care for children

Arch Dis Child, 84, 103-105 
Jöhr 2005

Keimer 1999

Klier 2003

Knapp 1995

Knapp et al. 2008

Koletzko 2004

Kuisma et al. 1995

Kumar et al. 1997

Miller 1990

Möller et al. 2002

Möller et al. 2005

Mönk 2003

Moront et al. 1996

Murdock et al. 1999
Jöhr M (2005): Kleine Kinder - große Probleme.

Anästh Intensivmed, $\underline{46}$, 358-372

Keimer R (1999): Therapie zerebraler Krampfanfälle im Kindesalter.

Notfall Rettungsmed, 2, 160-163

Klier J: Effizienzanalyse der Notarztsysteme im Rettungsdienstbereich Gießen: eine retrospektive Auswertung von Notarzteinsatzprotokollen über zwei Jahre vor und nah Einführung eines weiteren Notarztsystems (Rendezvoussystem) im Rettungsdienstbereich Gießen.

Med. Diss. Gießen 2004

Knapp JF (1995): A Call to Action: The Institute of Medicine Report on Emergency Medical Services for Children.

Pediatrics, $\underline{96}$, 173-174

Knapp J, Bernhard M, Hainer C, Sinkinger M, Brenner T, Schlechtriemen T, Gries A (2008): Besteht ein Zusammenhang zwischen der Einschätzung der Vitalgefährdung und der notfallmedizinischen Erfahrung des Notarztes?

Anaesthesist, 57, 1069-1074

Koletzko B: Kinderheilkunde und Jugendmedizin.

12. Auflage, Springer-Verlag Berlin, Heidelberg, New York, 2004

Kuisma M, Suominen P, Korpela R (1995): Paediatric out-of-hospital cardiac arrests - epidemiology and outcome.

Resuscitation, $\underline{30}$, 141-150

Kumar VR, Bachman DT, Kiskaddon RT (1997): Children and Adults in Cardiopulmonary Arrest: Are Advanced Life Support Guidelines Followed in the Prehospital Setting?

Ann Emerg Med, 29, 743-747

Miller GE (1990): The assessment of clinical skill/competence/performance.

Acad Emerg Med, 9, 1310-1318

Möller JC, Ballnus S, Kohl M, Göpel W, Barthel M, Krüger U, Friedrich HJ (2002): Evaluation of the performance of general emergency physicians in pediatric emergencies: Obstructive airway diseases, seizures, and trauma Pediatr Emerg Care, 18, 424-428

Möller JC, Geipel M, Steinetz O (2005): Kardiale Notfälle bei Kindern

Notfall Rettungsmed, $\underline{8}$, 422-428

Mönk S (2003): Die Bedeutung von Simulatoren.

Notfall Rettungsmed, $\underline{6}$, 37-39

Moront ML, Gotschall CS, Eichelberger MR (1996): Helicopter Transport of Injured Children: System Effectiveness nd Triage Criteria.

J Pediatr Surg, 31, 1183-1188

Murdock TC, Knapp JF, Dowd D, Campbell JP (1999): Bridging the Emergency Medical Services for Children Information Gap.

Arch Pediatr Adolesc Med, 153, 281-285 
Nagele und Kroesen 2000

Nakayama et al. 1989

Nicolai 2001

Ong et al. 2006

Pallin et al. 2008

Poets 2005

Reichmann et al. 1998

Richard et al. 2006

Roberts et al. 2005

Roessler und Zuzan 2006

Rossi 1999

Schlechtriemen et al. 2005 a)

Schlechtriemen et al. 2005 b)
Nagele P, Kroesen G (2000): Kindernotfälle im Notarztdienst.

Anaesthesist, $\underline{49}$, 725-731

Nakayama DK, Gardner MJ, Rowe MI (1989): Emergency Endotracheal Intubation in Pediatric Trauma.

Ann Surgery, 211, 218-223

Nicolai T (2001): Airwaymanagement bei Kindern in Notfallsituationen.

Monatsschr Kinderheilkd, 149, 466-469

Ong MEH, Stiell I, Osmond MH, Nesbitt L, Gerein R, Campbell S, McLellan B (2006): Etiology of pediatric out-of-hospital cardiac arrest by coroner's diagnosis.

Resuscitation, 68, 335-342

Pallin DJ, Goldstein JN, Moussally JS, Pelletier AJ, Green AR, Camargo CA

Jr. (2008): Seizure visits in US emergency departments: epidemiology and potential disparities in care.

Int J Emerg Med, 1, 97-105

Poets CF (2005): Der plötzliche Kindstod - Wissenwertes für den Notarzt.

Notfall Rettungsmed, $\underline{8}$, 533-538

Reichmann I, Aufmkolk M, Neudeck F, Bardenheuer M, Schmitt-Neuerburg KP (1998): Vergleich schwerer Mehrfachverletzungen im Kindes- und Erwachsenenalter.

Unfallchirurg, 101, 919-927

Richard J, Osmond MH, Nesbitt L, Stiell IG (2006): Management and outcomes of pediatric patients transported by emergency medical services in a Canadian prehospital system.

Can J Emerg Med, $\underline{8}$, 6-12,

Roberts K, Jewkes F, Whalley H, Hopkins D, Porter K (2005): A review of emergency equipment carried and procedures performed by UK front line paramedics on paediatric patients.

Emerg Med J, 22, 572-576

Roessler M, Zuzan O (2006): EMS systems in Germany.

Resuscitation, $\underline{68}$, 45-49

Rossi R (1999): Strategien zur Bewältigung von Notfällen im Kindesalter

Notfall Rettungsmed, 2, 31-34

Schlechtriemen T, Masson R, Burghofer K, Lackner C, Altemeyer K (2005):

Pädiatrische Notfälle in der präklinischen Notfallmedizin.

Anaesthesist, 55, 255-262

Schlechtriemen T, Burghofer K, Lackner CK, Altemeyer KH (2005): Validierung des NACA-Score anhand objektivierbarer Parameter.

Notfall Rettungsmed, 8, 96-108 
Schürmann und Lemburg 2001

Seidel 1986

Sirbaugh et al. 1999

Smith et al. 2005

Søreide et al. 2009

Statistisches Bundesamt

[DESTATIS] 2006

Stenke 2004

Stopfkuchen et al. 1999

Suominen et al. 1996

Svenson et al. 1996

Timmermann et al. 2005

Timmermann et al. 2007
Schürmann M, Lemburg P (2001): Pädiatrische intensiv-medizinische Transportversorgung. Verfahren und Probleme.

Notfall Rettungsmed, 4, 90-92

Seidel JS (1986): Emergency Medical Services and the Pediatric Patient: Are the Needs Being Met? II. Training and Equipping Emergency Medical Services Providers for Pediatric Emergencies.

Pediatrics, $\underline{78}$, 808-812

Sirbaugh PE, Pepe PE, Shook JE, Kimball KT, Goldman MJ, Ward MA, Mann DM (1999): AProspective, Population-Based Study of the Dempgraphics, Epidemiology, Management, and Outcome of Out-of-Hospital Pediatric Cardiopulmonary Arrest.

Ann Emerg Med, 33, 174-184

Smith R, Davis N, Bouamra O, Lecky F: The utilisation of intraosseus infusion in the rescucitation of paediatric major trauma patients.

Injury 2005, 36: 1034-1038; discussion 1039

Søreide K, Krüger AJ, Ellingsen CL, Tjosevik KE (2009): Pediatric trauma deaths are predominated by severe head injuries during spring and summer.

Scand J Trauma, Resusc Emerg Med, 17 ,3

Statistisches Buundesamt: www.destatis.de 2006

Stenke C: The Munich Paediatric Emergency Physician: 3667 paediatric emergencies from 1998-2001 as evaluated at two of four involved hospitals.

Med. Diss. München, 2004

Stopfkuchen H, Wollermann C, Krämer W (1999): Außerklinische Notfallsituationen (Notfälle/Akutfälle) im Kindesalter.

Notarzt, $\underline{15}$, 85-90

Suominen P, Silfvast T, Korpela R, Erosuo J (1996): Pediatric prehospital care provided by a physician-staffed emergency medical helicopter unit in Finland.

Pediatr Emerg Care, 12, 169-172

Svenson JE, Nypaver M, Calhoun R (1996): Pedatric prehospital care: Epidemiology of use in a predominantly rural state.

Pediatr Emerg Care, 12, 173-179

Timmermann A, Eich C, Nickel E, Russo S, Barwing J, Heuer JF, Braun U (2005): Simulation und Atemwegsmanagement.

Anaesthesist, 54, 582-587

Timmermann A, Eich C, Russo, SG, Barwing J, Hirn A, Rode H, Heuer JF, Heise D, Nickel E, Klockgether-Radke A, Graf BM (2007): Lehre und Simulation. Methoden, Anforderungen, Evaluation und Visionen

Anaesthesist, 투, 53-62 
Tsai und Kallsen 1987

Wang et al. 2006

Wantman und Chin 2003

Weiss et al. 2001

Weiss et al. 2009

Wheeler et al. 2003

Yee et al. 2005

Zink et al. 2004
Tsai A, Kallsen G (1987): Epidemiology of Pediatric Prehospital Care Ann Emerg Med, 16, 284-292

Wang HE, Lave JR, Sirio CA, Yealy DM (2006): Paramedic Intubation Errors: Isolated Events Or Symptoms Of Larger Problems?

Health Aff, 25, 501-509

Wantman A, Chin C (2003): Use of simulation in paediatric anaesthesia training.

Paediatr Anesth; $\underline{13}$, 749-753

Weiss M, Bernoulli L, Zollinger A (2001): Der NACA-Index. Aussagekraft und Stellenwert des modifizierten NACA-Indexes in der präklinischen Schweregraderfassung von Unfallpatienten.

Anaesthesist, $\underline{50}, 150-154$

Weiss M, Henz G, Eich C, Neuhaus D (2009): Intraossäre Infusion. Eine wichtige Technik auch für die Kinderanästhesie.

Anaesthesist, $\underline{58}$, 863-875

Wheeler DS, Clapp CR, Poss WB (2003): Training in pediatric critical care medicine: A survey of pediatric residency training programs.

Pediatr Emerg Care, 19, 1-5

Yee B, Naik VN, Joo HS, Savoldelli L, Chung DY, Houston PL Karatzoglou BJ, Hamstra SJ (2005): Nontechnical Skills in Anesthesia Crisis Management with Repeated Exposure to Simulation-based Education.

Anesthesiology 2005, 103, 241-248

Zink W, Bernhard M, Keul W, Martin E, Völkl A, Gries A (2004): Invasive Techniken in der Notfallmedizin.

Anaesthesist, $\underline{53}, 1086-1092$ 


\section{Lebenslauf}

Ich wurde am 20.03.1983 in Berlin geboren. Meine Schulausbildung erfolgte von 1989 bis 1995 an der 9. Grundschule in Berlin-Mitte. Von 1995 bis 2002 besuchte ich das Max-PlanckGymnasium in Berlin-Mitte. 2002 erlangte ich dort die allgemeine Hochschulreife (Abitur).

2002 begann ich an der Georg-August-Universität Göttingen das Studium der Humanmedizin und legte dort 2004 meine ärztliche Vorprüfung ab. Im Verlauf leistete ich Famulaturen in den Bereichen Unfallchirurgie, Notfallmedizin, Kardiologie, Gynäkologie, Gastroenterologie, Orthopädie und Urologie ab. Das Praktische Jahr absolvierte ich 2007 bis 2008 im Evangelischen Krankenhaus Göttingen-Weende, mit dem Wahlfach Urologie. Im November 2008 absolvierte ich die ärztliche Prüfung. Am 20.11.2008 wurde mir die ärztliche Approbation erteilt.

Am 15.01.2009 begann meine Weiterbildung zur Fachärztin für Unfallchirurgie und Orthopädie in der Klinik für Unfall- und Handchirurgie in den St. Vincentius-Kliniken in Karlsruhe (Chefarzt Dr. M. Rustemeier). 


\section{Danksagung}

Es ist mir ein Bedürfnis, mich an dieser Stelle bei Herrn Prof. Dr. med. M. Quintel für die freundliche Überlassung des Themas zu bedanken.

Ein herzlicher Dank gilt meinem Betreuer, Herrn Priv.-Doz. Dr. med. Ch. Eich, der mich in allen Phasen meiner Arbeit unterstützt, beraten und motiviert hat.

Bei Herrn Dr. rer. nat. B. Albrecht bedanke ich mich für die Unterstützung bei der Auswertung statistischer Daten.

Ein persönlicher Dank gilt meiner Familie für jegliche Art der Unterstützung. 